\title{
HIGHER-DIMENSIONAL GENERAL JACOBI IDENTITIES I
}

\author{
HIROKAZU NISHIMURA
}

\begin{abstract}
It was shown by the author [International Journal of Theoretical Physics 36 (1997), 1099-1131] that what is called the general Jacobi identity, obtaining in microcubes, underlies the Jacobi identity of vector fields. It is well known in the theory of Lie algebras that a plethora of higher-dimensional generalizations of the Jacobi identity hold, though they are usually established not as a derivation on the nose from the axioms of Lie algebras but by making an appeal to the socalled Poincaré-Birkhoff-Witt theorem and the like. The general Jacobi identity was rediscovered by Kirill Mackenzie in the second decade of this century [Geometric Methods in Physics, Birkhäuser/Springer, 2013, 357-366]. The principal objective of this paper is to investigate a four-dimensional generalization of the general Jacobi identity in detail. In a subsequent paper we will propose a uniform method for establishing a bevy of higher-dimensional generalizations of the general Jacobi identity under a single umbrella.
\end{abstract}

\section{INTRODUCTION}

It is known in synthetic differential geometry (cf. [2] and [4]) that vector fields on a microlinear space $M$ form a Lie algebra, for which the following antisymmetry holds:

$$
\left[X_{1}, X_{2}\right]+\left[X_{2}, X_{1}\right]=0
$$

It was shown in [3] that a somewhat deeper theorem below underlies the above identity.

Theorem 1.1. Let $M$ be a microlinear space. Given microsquares $\gamma_{12}, \gamma_{21}$ : $D^{2} \rightarrow M$ with $\gamma_{12}\left|D(2)=\gamma_{21}\right| D(2)$, we have

$$
\left(\gamma_{12}-\gamma_{21}\right)+\left(\gamma_{21}-\gamma_{12}\right)=0
$$

Now we consider the famous Jacobi identity

$$
\left[X_{1},\left[X_{2}, X_{3}\right]\right]+\left[X_{2},\left[X_{3}, X_{1}\right]\right]+\left[X_{3},\left[X_{1}, X_{2}\right]\right]=0 .
$$

It claims that the sum of $\left[X_{1},\left[X_{2}, X_{3}\right]\right]$ 's with the three cyclic permutations of $\{1,2,3\}$ applied vanishes. We note in passing that the three cyclic permutations of $\{1,2,3\}$ are no other than the three even permutations of $\{1,2,3\}$. It has been demonstrated in [6], [7], [8] and [10] that the following deeper theorem underlies the above identity.

$M S C$ (2010): primary 58A03, 18F $15,51 \mathrm{~K} 10$.

Keywords: general Jacobi identity, synthetic differential geometry, Frölicher-Nijenhuis calculus. 
Theorem 1.2. (General Jacobi identity) Let $M$ be a microlinear space. Given microcubes $\gamma_{123}, \gamma_{132}, \gamma_{213}, \gamma_{231}, \gamma_{312}, \gamma_{321}: D^{3} \rightarrow M$ with

$$
\begin{aligned}
& \gamma_{123}\left|\left\{\left(d_{1}, d_{2}, d_{3}\right) \in D^{3} \mid d_{2} d_{3}=0\right\}=\gamma_{132}\right|\left\{\left(d_{1}, d_{2}, d_{3}\right) \in D^{3} \mid d_{2} d_{3}=0\right\}, \\
& \gamma_{231}\left|\left\{\left(d_{1}, d_{2}, d_{3}\right) \in D^{3} \mid d_{2} d_{3}=0\right\}=\gamma_{321}\right|\left\{\left(d_{1}, d_{2}, d_{3}\right) \in D^{3} \mid d_{2} d_{3}=0\right\}, \\
& \gamma_{231}\left|\left\{\left(d_{1}, d_{2}, d_{3}\right) \in D^{3} \mid d_{1} d_{3}=0\right\}=\gamma_{213}\right|\left\{\left(d_{1}, d_{2}, d_{3}\right) \in D^{3} \mid d_{1} d_{3}=0\right\}, \\
& \gamma_{312}\left|\left\{\left(d_{1}, d_{2}, d_{3}\right) \in D^{3} \mid d_{1} d_{3}=0\right\}=\gamma_{132}\right|\left\{\left(d_{1}, d_{2}, d_{3}\right) \in D^{3} \mid d_{1} d_{3}=0\right\}, \\
& \gamma_{312}\left|\left\{\left(d_{1}, d_{2}, d_{3}\right) \in D^{3} \mid d_{1} d_{2}=0\right\}=\gamma_{321}\right|\left\{\left(d_{1}, d_{2}, d_{3}\right) \in D^{3} \mid d_{1} d_{2}=0\right\}, \\
& \gamma_{123}\left|\left\{\left(d_{1}, d_{2}, d_{3}\right) \in D^{3} \mid d_{1} d_{2}=0\right\}=\gamma_{213}\right|\left\{\left(d_{1}, d_{2}, d_{3}\right) \in D^{3} \mid d_{1} d_{2}=0\right\},
\end{aligned}
$$

we have

$$
\begin{aligned}
\left(\left(\gamma_{123} \frac{\dot{1}}{1} \gamma_{132}\right)-\left(\gamma_{231} \frac{\dot{-}}{1} \gamma_{321}\right)\right) & +\left(\left(\gamma_{231} \frac{\dot{-}}{2} \gamma_{213}\right)-\left(\gamma_{312}-\gamma_{132}\right)\right) \\
& +\left(\left(\gamma_{312} \frac{\dot{ }}{3} \gamma_{321}\right)-\left(\gamma_{123} \frac{\dot{-}}{3} \gamma_{213}\right)\right)=0 .
\end{aligned}
$$

The general Jacobi identity was rediscovered by Kirill Mackenzie [5] in a somewhat different context. We add that the general Jacobi identity plays a fundamental role in a combinatorial or geometric proof of Jacobi-like identities in the so-called Frölicher-Nijenhuis calculus (cf. [9]).

Now we consider the following four-dimensional analogue of the Jacobi identity.

$$
\begin{aligned}
& {\left[X_{1},\left[X_{2},\left[X_{3}, X_{4}\right]\right]\right]+\left[X_{1},\left[X_{3},\left[X_{4}, X_{2}\right]\right]\right]+\left[X_{1},\left[X_{4},\left[X_{2}, X_{3}\right]\right]\right]+} \\
& {\left[X_{2},\left[X_{1},\left[X_{4}, X_{3}\right]\right]\right]+\left[X_{2},\left[X_{3},\left[X_{1}, X_{4}\right]\right]\right]+\left[X_{2},\left[X_{4},\left[X_{3}, X_{1}\right]\right]\right]+} \\
& {\left[X_{3},\left[X_{1},\left[X_{2}, X_{4}\right]\right]\right]+\left[X_{3},\left[X_{2},\left[X_{4}, X_{1}\right]\right]\right]+\left[X_{3},\left[X_{4},\left[X_{1}, X_{2}\right]\right]\right]+} \\
& {\left[X_{4},\left[X_{1},\left[X_{3}, X_{2}\right]\right]\right]+\left[X_{4},\left[X_{2},\left[X_{1}, X_{3}\right]\right]\right]+\left[X_{4},\left[X_{3},\left[X_{2}, X_{1}\right]\right]\right]=0 .}
\end{aligned}
$$

It claims that the sum of $\left[X_{1},\left[X_{2},\left[X_{3}, X_{4}\right]\right]\right]$ 's with the twelve even permutations of $\{1,2,3,4\}$ applied vanishes.

The principal objective in this paper is to establish a four-dimensional version of the general Jacobi identity underpinning the above identity (1.1). Below we will discuss a slew of higher-dimensional general Jacobi identities underlying the higher-dimensional Jacobi identities discussed in [1] and [12] (the former called them generalized Jacobi identities) from a coherent standpoint. For a good introduction to generalized Jacobi identities, the reader is referred to Chapter 8 of [11]. We know well that various higher-dimensional Jacobi identities are logical consequences of the three-dimensional Jacobi identity, but we guess that higherdimensional general Jacobi identities are by no means logical consequences of the three-dimensional general Jacobi identity. We assume the reader to be familiar with [4] up to Chapter 3.

\section{Strong DifFERENCES}

First we introduce the notion of a simplicial small object after [6], though in a somewhat generalized form. 
Notation 2.1. (Simplicial small objects) Let $n$ be a natural number. Given a subset $\mathfrak{p}$ of

$$
\{(i, j) \in \mathbb{N} \times \mathbb{N} \mid 1 \leq i \leq n, 1 \leq j \leq n, i \neq j\}
$$

and a subset $\xi$ of

$$
\{i \in \mathbb{N} \mid 1 \leq i \leq n\}
$$

$D^{n}\{\mathfrak{p}, \xi\}$ denotes the set

$$
\left\{\left(d_{1}, \ldots, d_{n}\right): D^{n} \mid d_{i} d_{j}=0 \text { for any }(i, j) \in \mathfrak{p}, d_{i}=0 \text { for any } i \in \xi\right\},
$$

which is surely a small object. By way of an example, we have

$$
\begin{aligned}
& D(2)=D^{2}\{(1,2)\}, \\
& D(3)=D^{3}\{(1,2),(1,3),(2,3)\}
\end{aligned}
$$

and $D^{3}\{1,3\}$ can be identified with $D$ via the canonical isomorphism

$$
d \in D \mapsto(0, d, 0) \in D^{3}\{1,3\} .
$$

The notion of strong difference in synthetic differential geometry is based upon the following lemma.

Lemma 2.2. (cf. the first Lemma in $\S 3.4$ of [4]) The diagram

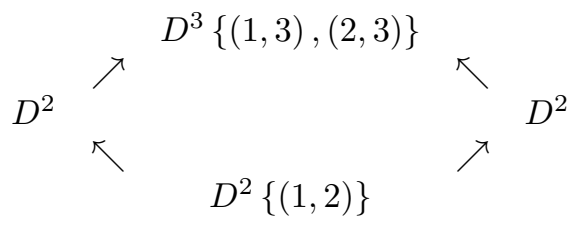

with the lower two arrows being the canonical injections and the upper two arrows being

$$
\begin{aligned}
& j_{1}^{2}:\left(d_{1}, d_{2}\right) \in D^{2} \mapsto\left(d_{1}, d_{2}, d_{1} d_{2}\right) \in D^{3}\{(1,3),(2,3)\}, \\
& j_{2}^{2}:\left(d_{1}, d_{2}\right) \in D^{2} \mapsto\left(d_{1}, d_{2}, 0\right) \in D^{3}\{(1,3),(2,3)\}
\end{aligned}
$$

from left to right is a quasi-colimit diagram.

Corollary 2.3. Let $M$ be a microlinear space with two microsquares $\gamma_{1}, \gamma_{2}$ : $D^{2} \rightarrow M$ abiding by

$$
\gamma_{1}\left|D^{2}\{(1,2)\}=\gamma_{2}\right| D^{2}\{(1,2)\} .
$$

Then, there exists a unique mapping

$$
\mathfrak{n}_{\left(\gamma_{1}, \gamma_{2}\right)}^{2}: D^{3}\{(1,3),(2,3)\} \rightarrow M
$$

such that $\mathfrak{n}_{\left(\gamma_{1}, \gamma_{2}\right)}^{2} \circ j_{1}^{2}=\gamma_{1}$ and $\mathfrak{n}_{\left(\gamma_{1}, \gamma_{2}\right)}^{2} \circ j_{2}^{2}=\gamma_{2}$.

Notation 2.4. In the above notation, in Corollary 2.3 we write $\gamma_{1}-\gamma_{2}$ for the mapping

$$
d \in D \mapsto \mathfrak{n}_{\left(\gamma_{1}, \gamma_{2}\right)}^{2}(0,0, d)
$$

The notion of strong difference can easily be relativized. 
Lemma 2.5. Let $n$ be a natural number. The diagram

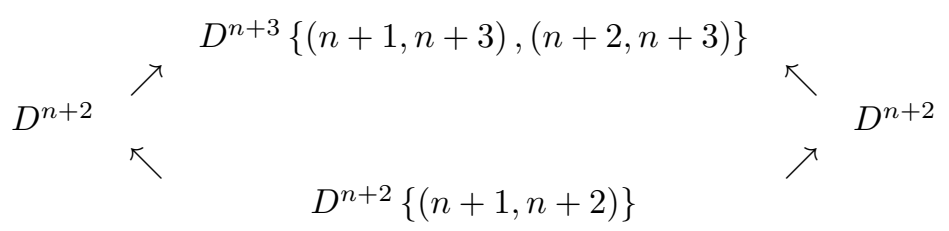

with the lower two arrows being the canonical injections and the upper two arrows being

$$
\begin{aligned}
j_{1}^{n+2}:\left(d_{1}, \ldots, d_{n}, d_{n+1}, d_{n+2}\right) \in D^{n+2} & \mapsto\left(d_{1}, \ldots, d_{n}, d_{n+1}, d_{n+2}, d_{n+1} d_{n+2}\right) \in \\
& \in D^{n+3}\{(n+1, n+3),(n+2, n+3)\}, \\
j_{2}^{n+2}:\left(d_{1}, \ldots, d_{n}, d_{n+1}, d_{n+2}\right) \in D^{n+2} & \mapsto\left(d_{1}, \ldots, d_{n}, d_{n+1}, d_{n+2}, 0\right) \in \\
& \in D^{n+3}\{(n+1, n+3),(n+2, n+3)\}
\end{aligned}
$$

from left to right is a quasi-colimit diagram.

Corollary 2.6. Let $n$ be a natural number. Let $M$ be a microlinear space with two mappings $\gamma_{1}, \gamma_{2}: D^{n+2} \rightarrow M$ abiding by

$$
\gamma_{1}\left|D^{n+2}\{(n+1, n+2)\}=\gamma_{2}\right| D^{n+2}\{(n+1, n+2)\} .
$$

Then, there exists a unique mapping

$$
\mathfrak{n}_{\left(\gamma_{1}, \gamma_{2}\right)}^{n+2}: D^{n+3}\{(n+1, n+3),(n+2, n+3)\} \rightarrow M
$$

such that $\mathfrak{n}_{\left(\gamma_{1}, \gamma_{2}\right)}^{n+2} \circ j_{1}^{n+2}=\gamma_{1}$ and $\mathfrak{n}_{\left(\gamma_{1}, \gamma_{2}\right)}^{n+2} \circ j_{2}^{3}=\gamma_{2}$.

Notation 2.7. Let $n$ be a natural number. Let $M$ be a microlinear space. Given $\gamma: D^{n} \rightarrow M$ and a permutation $\sigma$ of $\{1, \ldots, n\}$, we write $\gamma^{\sigma}$ for the mapping

$$
\left(d_{1}, \ldots, d_{n}\right) \in D^{n} \mapsto \gamma\left(d_{\sigma^{-1}(1)}, \ldots, d_{\sigma^{-1}(n)}\right) \in M .
$$

Notation 2.8. Let $n$ be a natural number. Let $M$ be a microlinear space.

(1) Given $\gamma_{1}, \gamma_{2}: D^{n+2} \rightarrow M$ with

$$
\gamma_{1}\left|D^{n+2}\{(n+1, n+2)\}=\gamma_{2}\right| D^{n+2}\{(n+1, n+2)\}
$$

we write $\gamma_{1} \underset{1 \ldots n}{-} \gamma_{2}$ for the mapping

$$
\left(d_{1}, \ldots, d_{n}, d_{n+1}\right) \in D^{n+1} \mapsto \mathfrak{n}_{\left(\gamma_{1}, \gamma_{2}\right)}^{n+2}\left(d_{1}, \ldots, d_{n}, 0,0, d_{n+1}\right) \in M .
$$

(2) Given a permutation $\sigma$ of $\{1, \ldots, n, n+1, n+2\}$ and $\gamma_{1}, \gamma_{2}: D^{n+2} \rightarrow M$ with

$$
\gamma_{1}\left|D^{n+2}\{(\sigma(n+1), \sigma(n+2))\}=\gamma_{2}\right| D^{n+2}\{(\sigma(n+1), \sigma(n+2))\}
$$

we write $\gamma_{1} \underset{\sigma(1) \ldots \sigma(n)}{-} \gamma_{2}$ for $\left(\gamma_{1}\right)^{\sigma} \underset{1 \ldots n}{-}\left(\gamma_{2}\right)^{\sigma}$.

The following result is well known. 
Lemma 2.9. ( cf. Proposition 6 in $\S 2.2$ of [4]) The diagram

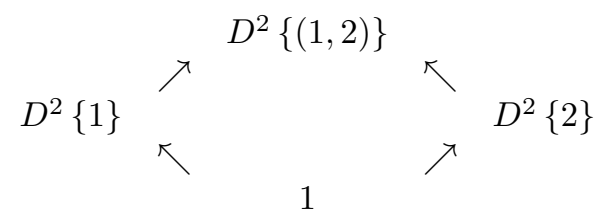

with the four arrows being the canonical injections is a quasi-colimit diagram.

Corollary 2.10. Let $M$ be a microlinear space. Given $\gamma_{1}, \gamma_{2}: D^{2} \rightarrow M$,

$$
\gamma_{1}\left|D^{2}\{(1,2)\}=\gamma_{2}\right| D^{2}\{(1,2)\}
$$

is obtained iff both

$$
\gamma_{1}\left|D^{2}\{1\}=\gamma_{2}\right| D^{2}\{1\}
$$

and

obtain.

$$
\gamma_{1}\left|D^{2}\{2\}=\gamma_{2}\right| D^{2}\{2\}
$$

It can readily be relativized and generalized.

Lemma 2.11. The diagram

$$
\begin{aligned}
& D^{n+m_{1}+m_{2}} \\
& \left\{\begin{array}{c}
\left(n+i, n+m_{1}+j\right) \\
1 \leq i \leq m_{1}, \\
1 \leq j \leq m_{2}
\end{array}\right\} \\
& D^{n+m_{1}+m_{2}} \\
& \left\{\begin{array}{c}
n+1, \ldots, \\
n+m_{1}
\end{array}\right\}
\end{aligned}
$$
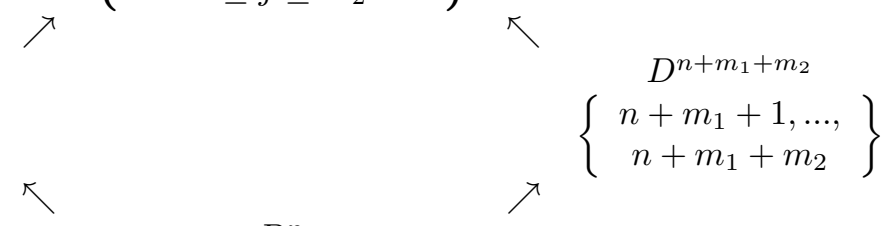

with the four arrows being the canonical injections is a quasi-colimit diagram.

Corollary 2.12. Let $M$ be a microlinear space. Given $\gamma_{1}, \gamma_{2}: D^{n+m_{1}+m_{2}} \rightarrow$ $M$

$$
\begin{aligned}
\gamma_{1} \mid D^{n+m_{1}+m_{2}}\left\{\left(n+i, n+m_{1}+j\right) \mid 1 \leq i \leq m_{1}, 1 \leq j \leq m_{2}\right\} \\
=\gamma_{2} \mid D^{n+m_{1}+m_{2}}\left\{\left(n+i, n+m_{1}+j\right) \mid 1 \leq i \leq m_{1}, 1 \leq j \leq m_{2}\right\}
\end{aligned}
$$

is obtained iff both

$$
\gamma_{1}\left|D^{n+m_{1}+m_{2}}\left\{n+1, \ldots, n+m_{1}\right\}=\gamma_{2}\right| D^{n+m_{1}+m_{2}}\left\{n+1, \ldots, n+m_{1}\right\}
$$

and

$$
\begin{aligned}
\gamma_{1} \mid D^{n+m_{1}+m_{2}}\left\{n+m_{1}+1\right. & \left., \ldots, n+m_{1}+m_{2}\right\} \\
& =\gamma_{2} \mid D^{n+m_{1}+m_{2}}\left\{n+m_{1}+1, \ldots, n+m_{1}+m_{2}\right\}
\end{aligned}
$$

are obtained.

Proposition 2.13. Let $M$ be a microlinear space. Then, we have the following two statements: 
(1) Given

$$
\gamma_{1}: D^{4} \rightarrow M, \gamma_{2}: D^{4} \rightarrow M, \gamma_{3}: D^{4} \rightarrow M, \gamma_{4}: D^{4} \rightarrow M
$$

if it holds that

$$
\begin{aligned}
& \gamma_{1}\left|D^{4}\{(3,4)\}=\gamma_{2}\right| D^{4}\{(3,4)\}, \\
& \gamma_{3}\left|D^{4}\{(3,4)\}=\gamma_{4}\right| D^{4}\{(3,4)\} \\
& \gamma_{1}\left|D^{4}\{(2,3),(2,4)\}=\gamma_{3}\right| D^{4}\{(2,3),(2,4)\}, \\
& \gamma_{2}\left|D^{4}\{(2,3),(2,4)\}=\gamma_{4}\right| D^{4}\{(2,3),(2,4)\},
\end{aligned}
$$

Then, all of

$$
\gamma_{1}-\gamma_{12}, \quad \gamma_{3} \underset{12}{-} \gamma_{4}, \quad\left(\gamma_{1}-\gamma_{12}\right)-\left(\gamma_{3}-\gamma_{12}\right)
$$

are well defined.

(2) Given

$$
\begin{aligned}
& \gamma_{1}: D^{4} \rightarrow M, \gamma_{2}: D^{4} \rightarrow M, \gamma_{3}: D^{4} \rightarrow M, \gamma_{4}: D^{4} \rightarrow M, \\
& \gamma_{5}: D^{4} \rightarrow M, \gamma_{6}: D^{4} \rightarrow M, \gamma_{7}: D^{4} \rightarrow M, \gamma_{8}: D^{4} \rightarrow M
\end{aligned}
$$

if it holds that

$$
\begin{aligned}
& \gamma_{1}\left|D^{4}\{(3,4)\}=\gamma_{2}\right| D^{4}\{(3,4)\}, \\
& \gamma_{3}\left|D^{4}\{(3,4)\}=\gamma_{4}\right| D^{4}\{(3,4)\}, \\
& \gamma_{5}\left|D^{4}\{(3,4)\}=\gamma_{6}\right| D^{4}\{(3,4)\}, \\
& \gamma_{7}\left|D^{4}\{(3,4)\}=\gamma_{8}\right| D^{4}\{(3,4)\}, \\
& \gamma_{1}\left|D^{4}\{(2,3),(2,4)\}=\gamma_{3}\right| D^{4}\{(2,3),(2,4)\}, \\
& \gamma_{2}\left|D^{4}\{(2,3),(2,4)\}=\gamma_{4}\right| D^{4}\{(2,3),(2,4)\}, \\
& \gamma_{5}\left|D^{4}\{(2,3),(2,4)\}=\gamma_{7}\right| D^{4}\{(2,3),(2,4)\}, \\
& \gamma_{6}\left|D^{4}\{(2,3),(2,4)\}=\gamma_{8}\right| D^{4}\{(2,3),(2,4)\}, \\
& \gamma_{1}\left|D^{4}\{(1,2),(1,3),(1,4)\}=\gamma_{5}\right| D^{4}\{(1,2),(1,3),(1,4)\}, \\
& \gamma_{2}\left|D^{4}\{(1,2),(1,3),(1,4)\}=\gamma_{6}\right| D^{4}\{(1,2),(1,3),(1,4)\}, \\
& \gamma_{3}\left|D^{4}\{(1,2),(1,3),(1,4)\}=\gamma_{7}\right| D^{4}\{(1,2),(1,3),(1,4)\}, \\
& \gamma_{4}\left|D^{4}\{(1,2),(1,3),(1,4)\}=\gamma_{8}\right| D^{4}\{(1,2),(1,3),(1,4)\},
\end{aligned}
$$

Then, all of

$$
\begin{aligned}
& \gamma_{1}-\gamma_{12}, \quad \gamma_{3}-\gamma_{12}, \quad \gamma_{5}-\gamma_{6}, \quad \gamma_{7}-\gamma_{12}
\end{aligned}
$$

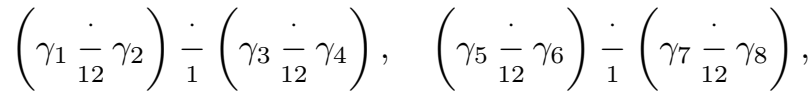

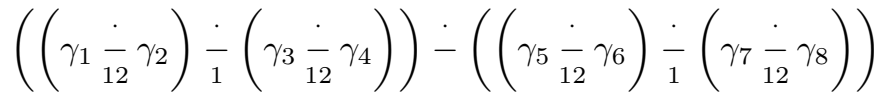

are well defined.

Proof. We deal with the above two statements in order. 
(1) For the first statement, we have to show that

$$
\left(\gamma_{1}-\gamma_{12}\right)\left|D^{3}\{(2,3)\}=\left(\gamma_{3} \frac{-}{12} \gamma_{4}\right)\right| D^{3}\{(2,3)\}
$$

which is, by dint of Corollary 2.12, equivalent to showing that

$$
\begin{aligned}
& \left(\gamma_{1}-\gamma_{12}\right)\left|D^{3}\{2\}=\left(\gamma_{3}-\gamma_{12}\right)\right| D^{3}\{2\}, \\
& \left(\gamma_{1}-\gamma_{12}\right)\left|D^{3}\{3\}=\left(\gamma_{3} \frac{\cdot}{12} \gamma_{4}\right)\right| D^{3}\{3\}
\end{aligned}
$$

because of the quasi-colimit diagram

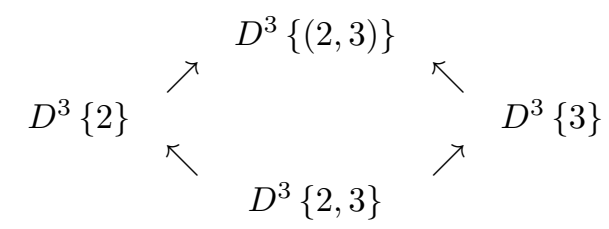

with the four arrows being the canonical injections (Lemma 2.11 with $\left.n=m_{1}=m_{2}=1\right)$. Due to the quasi-colimit diagram

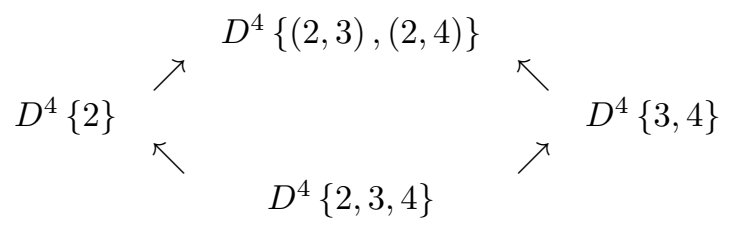

with the four arrows being the canonical injections (Lemma 2.11 with $n=$ $m_{1}=1$ and $m_{2}=2$ ), the condition (2.2) is equivalent to the conditions

$$
\begin{aligned}
& \gamma_{1}\left|D^{4}\{2\}=\gamma_{3}\right| D^{4}\{2\}, \\
& \gamma_{1}\left|D^{4}\{3,4\}=\gamma_{3}\right| D^{4}\{3,4\}
\end{aligned}
$$

while the condition (2.3) is equivalent to the conditions

$$
\begin{aligned}
& \gamma_{2}\left|D^{4}\{2\}=\gamma_{4}\right| D^{4}\{2\}, \\
& \gamma_{2}\left|D^{4}\{3,4\}=\gamma_{4}\right| D^{4}\{3,4\} .
\end{aligned}
$$

In order to show that (2.8) is obtained, we note that the quasi-colimit diagram in (2.1) with $n=2$ is to be restricted to the quasi-colimit diagram

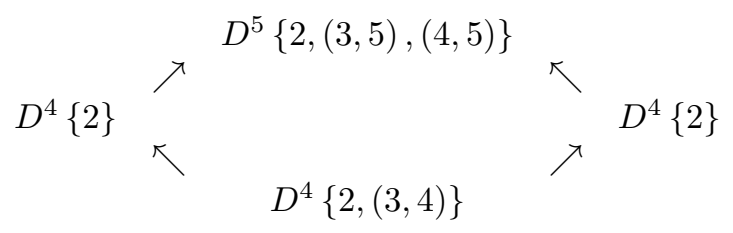

so that the conditions (2.10) and (2.12) imply (2.8). It is easy to see that

$$
\begin{aligned}
& \left(\left(\gamma_{1}-\gamma_{2}\right) \mid D^{3}\{3\}\right) \circ i_{2}^{3}=\left(\gamma_{1} \mid D^{4}\{3,4\}\right) \circ i_{2}^{4}=\left(\gamma_{2} \mid D^{4}\{3,4\}\right) \circ i_{2}^{4}, \\
& \left(\left(\gamma_{3} \frac{-}{12} \gamma_{4}\right) \mid D^{3}\{3\}\right) \circ i_{2}^{3}=\left(\gamma_{3} \mid D^{4}\{3,4\}\right) \circ i_{2}^{4}=\left(\gamma_{4} \mid D^{4}\{3,4\}\right) \circ i_{2}^{4}
\end{aligned}
$$


are obtained with

$$
\begin{aligned}
& i_{2}^{3}:\left(d_{1}, d_{2}\right) \in D^{2} \mapsto\left(d_{1}, d_{2}, 0\right) \in D^{3}, \\
& i_{2}^{4}:\left(d_{1}, d_{2}\right) \in D^{2} \mapsto\left(d_{1}, d_{2}, 0,0\right) \in D^{4}
\end{aligned}
$$

so that (2.11) and (2.13) imply (2.9).

(2) For the second statement, we have to show that

$$
\begin{aligned}
&\left(\left(\gamma_{1}-\frac{\dot{1}}{12} \gamma_{2}\right)-\left(\gamma_{3} \frac{\dot{-}}{12} \gamma_{4}\right)\right) \mid D^{2}\{(1,2)\}= \\
&=\left(\left(\gamma_{5} \frac{\dot{1}}{12} \gamma_{6}\right)-\left(\gamma_{7} \frac{\dot{-}}{12} \gamma_{8}\right)\right) \mid D^{2}\{(1,2)\}
\end{aligned}
$$

which is equivalent to showing that

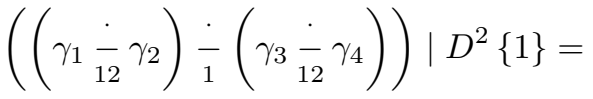

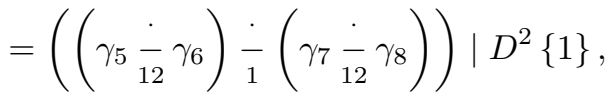

$$
\begin{aligned}
& \left(\left(\gamma_{1} \frac{\cdot}{12} \gamma_{2}\right) \div\left(\gamma_{3}-\gamma_{12}\right)\right) \mid D^{2}\{2\}= \\
& =\left(\left(\gamma_{5}-\gamma_{12}\right)-\frac{\cdot}{1}\left(\gamma_{7}-\gamma_{12}\right)\right) \mid D^{2}\{2\}
\end{aligned}
$$

because of the quasi-colimit diagram

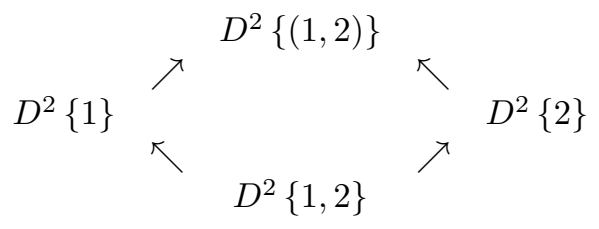

with the four arrows being the canonical injections (Lemma 2.9). Due to the quasi-colimit diagram

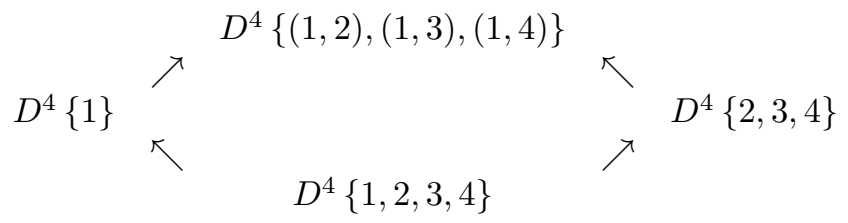

with the four arrows being the canonical injections (Lemma 2.11 with $n=0, m_{1}=1$ and $m_{2}=3$ ), we have

- the condition (2.4) is equivalent to the conditions

$$
\begin{aligned}
\gamma_{1} \mid D^{4}\{1\} & =\gamma_{5} \mid D^{4}\{1\}, \\
\gamma_{1} \mid D^{4}\{2,3,4\} & =\gamma_{5} \mid D^{4}\{2,3,4\},
\end{aligned}
$$

- the condition (2.5) is equivalent to the conditions

$$
\gamma_{2}\left|D^{4}\{1\}=\gamma_{6}\right| D^{4}\{1\},
$$




$$
\gamma_{2}\left|D^{4}\{2,3,4\}=\gamma_{6}\right| D^{4}\{2,3,4\},
$$

- the condition (2.6) is equivalent to the conditions

$$
\begin{aligned}
\gamma_{3} \mid D^{4}\{1\} & =\gamma_{7} \mid D^{4}\{1\}, \\
\gamma_{3} \mid D^{4}\{2,3,4\} & =\gamma_{7} \mid D^{4}\{2,3,4\},
\end{aligned}
$$

- the condition (2.7) is equivalent to the conditions

$$
\begin{aligned}
\gamma_{4} \mid D^{4}\{1\} & =\gamma_{8} \mid D^{4}\{1\}, \\
\gamma_{4} \mid D^{4}\{2,3,4\} & =\gamma_{8} \mid D^{4}\{2,3,4\} .
\end{aligned}
$$

In order to show that (2.14) is obtained, we note first that the quasicolimit diagram in 2.1 with $n=2$ is to be restricted to the quasi-colimit diagram

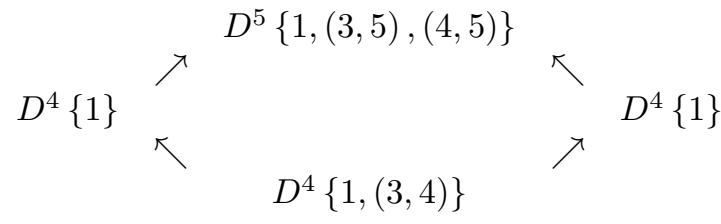

so that we have

- the conditions (2.16) and (2.18) imply

$$
\left(\gamma_{1}-\gamma_{12}\right)\left|D^{3}\{1\}=\left(\gamma_{5} \frac{\cdot}{12} \gamma_{6}\right)\right| D^{3}\{1\}
$$

- the conditions (2.20) and (2.22) imply

$$
\left(\gamma_{3}-\gamma_{12}\right)\left|D^{3}\{1\}=\left(\gamma_{7} \frac{\cdot}{12} \gamma_{8}\right)\right| D^{3}\{1\}
$$

We note also that the quasi-colimit diagram in (2.1) with $n=1$ is to be restricted to the quasi-colimit diagram

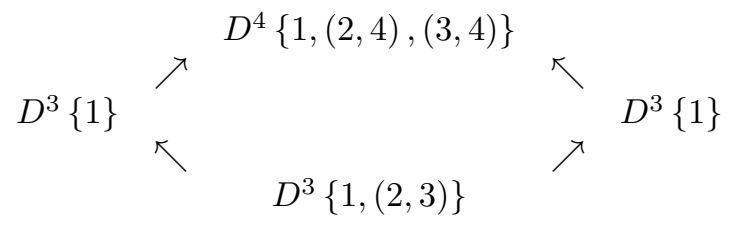

so that the conditions (2.24) and (2.25) imply the condition (2.14). It is easy to see that

$$
\begin{aligned}
& \left(\left(\left(\gamma_{1}-\gamma_{2}\right)-\left(\gamma_{3}-\gamma_{4}\right)\right) \mid D^{2}\{2\}\right) \circ i^{2} \\
& =\left(\left(\gamma_{1}-\gamma_{2}\right) \mid D^{3}\{2,3\}\right) \circ i^{3}=\left(\left(\gamma_{3}-\gamma_{4}\right) \mid D^{3}\{2,3\}\right) \circ i^{3} \\
& =\left(\gamma_{1} \mid D^{4}\{2,3,4\}\right) \circ i^{4}=\left(\gamma_{2} \mid D^{4}\{2,3,4\}\right) \circ i^{4} \\
& =\left(\gamma_{3} \mid D^{4}\{2,3,4\}\right) \circ i^{4}=\left(\gamma_{4} \mid D^{4}\{2,3,4\}\right) \circ i^{4}
\end{aligned}
$$


and

$$
\begin{aligned}
& \left(\left(\left(\gamma_{5} \frac{\dot{1}}{12} \gamma_{6}\right)-\left(\gamma_{7}-\gamma_{8}\right)\right) \mid D^{2}\{2\}\right) \circ i^{2} \\
& =\left(\left(\gamma_{5} \frac{-}{12} \gamma_{6}\right) \mid D^{3}\{2,3\}\right) \circ i^{3}=\left(\left(\gamma_{7} \frac{\dot{1}}{12} \gamma_{8}\right) \mid D^{3}\{2,3\}\right) \circ i^{3} \\
& =\left(\gamma_{5} \mid D^{4}\{2,3,4\}\right) \circ i^{4}=\left(\gamma_{6} \mid D^{4}\{2,3,4\}\right) \circ i^{4} \\
& =\left(\gamma_{7} \mid D^{4}\{2,3,4\}\right) \circ i^{4}=\left(\gamma_{8} \mid D^{4}\{2,3,4\}\right) \circ i^{4}
\end{aligned}
$$

are obtained with

$$
\begin{aligned}
& i^{2}: d \in D \mapsto(d, 0) \in D^{2}\{2\}, \\
& i^{3}: d \in D \mapsto(d, 0,0) \in D^{3}\{2,3\}, \\
& i^{4}: d \in D \mapsto(d, 0,0,0) \in D^{4}\{2,3,4\}
\end{aligned}
$$

so that (2.17), (2.19), (2.21) and (2.23) imply (2.15).

Notation 2.14. Let $M$ be a microlinear space.

(1) We denote by $\mathfrak{X}(M)$ the totality of vector fields on $M$. It forms a Lie algebra. We take the third viewpoint of a vector field in the essentially equivalent three discussed in $\S 3.2$ of [4]. Namely, a vector field $X$ on $M$ is a mapping $d \in D \mapsto X_{d} \in M^{M}$ with $X_{0}=\operatorname{id}_{M}$.

(2) Given $X, \ldots, X_{n} \in \mathfrak{X}(M)$, we denote by $X_{n} * \ldots * X_{1}$ the mapping $\left(d_{1}, \ldots, d_{n}\right) \in D^{n} \mapsto\left(X_{n}\right)_{d_{n}} \circ \ldots \circ\left(X_{1}\right)_{d_{1}} \in M^{M}$.

(3) We recall (cf. Proposition 8 in $\S 3.4$ of [4]) that

$$
\left[X_{1}, X_{2}\right]=X_{2} * X_{1}-\left(X_{1} * X_{2}\right)^{\sigma_{21}}
$$

with

$$
\sigma_{21}=\left(\begin{array}{c}
12 \\
21
\end{array}\right) .
$$

We recall (cf. Proposition 2.7 of [6]) that

$$
\begin{aligned}
{\left[X_{1},\left[X_{2}, X_{3}\right]\right]=\left(X_{3} * X_{2} * X_{1} \frac{\dot{1}}{1}\left(X_{2} * X_{3} * X_{1}\right)^{\sigma_{132}}\right)-} & \\
& \left(\left(X_{1} * X_{3} * X_{2}\right)^{\sigma_{231}} \frac{\dot{-}}{1}\left(X_{1} * X_{2} * X_{3}\right)^{\sigma_{321}}\right)
\end{aligned}
$$

with

$$
\sigma_{132}=\left(\begin{array}{c}
123 \\
132
\end{array}\right), \quad \sigma_{231}=\left(\begin{array}{l}
123 \\
312
\end{array}\right), \quad \sigma_{321}=\left(\begin{array}{c}
123 \\
321
\end{array}\right) .
$$

We note that

$$
\left.\begin{array}{l}
{\left[X_{1},\left[X_{2},\left[X_{3}, X_{4}\right]\right]\right]} \\
=\left(\begin{array}{c}
\left(X_{4} * X_{3} * X_{2} * X_{1} \frac{\dot{-}}{12}\left(X_{3} * X_{4} * X_{2} * X_{1}\right)^{\sigma_{1243}}\right) \frac{-}{1} \\
\left(\left(X_{2} * X_{4} * X_{3} * X_{1}\right)^{\sigma_{1342}} \frac{\dot{-}}{12}\left(X_{2} * X_{3} * X_{4} * X_{1}\right)^{\sigma_{1432}}\right.
\end{array}\right)
\end{array}\right) .
$$




$$
\left(\begin{array}{c}
\left(\left(X_{1} * X_{4} * X_{3} * X_{2}\right)^{\sigma_{2341}} \frac{\dot{-}}{12}\left(X_{1} * X_{3} * X_{4} * X_{2}\right)^{\sigma_{2431}}\right) \frac{-}{1} \\
\left(\left(X_{1} * X_{2} * X_{4} * X_{3}\right)^{\sigma_{3421}} \frac{\dot{\sim}}{12}\left(X_{1} * X_{2} * X_{3} * X_{4}\right)^{\sigma_{4321}}\right)
\end{array}\right)
$$

with

$$
\begin{aligned}
& \sigma_{1243}=\left(\begin{array}{l}
1234 \\
1243
\end{array}\right), \sigma_{1342}=\left(\begin{array}{l}
1234 \\
1423
\end{array}\right), \sigma_{1432}=\left(\begin{array}{l}
1234 \\
1432
\end{array}\right), \sigma_{2341}=\left(\begin{array}{l}
1234 \\
4123
\end{array}\right), \\
& \sigma_{2431}=\left(\begin{array}{l}
1234 \\
4132
\end{array}\right), \sigma_{3421}=\left(\begin{array}{l}
1234 \\
4312
\end{array}\right), \sigma_{4321}=\left(\begin{array}{l}
1234 \\
4321
\end{array}\right) .
\end{aligned}
$$

\section{A FOUR-DIMENSIONAL GENERAL JACOBI IDENTITY}

Theorem 3.1. The diagram whose underlying directed graph consists of vertices

$$
\begin{aligned}
& P, Q^{1234}, Q^{1243}, Q^{1324}, Q^{1342}, Q^{1423}, Q^{1432}, Q^{2134}, Q^{2143}, Q^{2314}, Q^{2341}, Q^{2413}, Q^{2431}, \\
& Q^{3124}, Q^{3142}, Q^{3214}, Q^{3241}, Q^{3412}, Q^{3421}, Q^{4123}, Q^{4132}, Q^{4213}, Q^{4231}, Q^{4312}, Q^{4321}, \\
& R_{12}^{1234,1243}, R_{12}^{1342,1432}, R_{12}^{2341,2431}, R_{12}^{3421,4321}, R_{12}^{2134,2143}, R_{12}^{3412,4312}, R_{13}^{1324,1342}, \\
& R_{13}^{1243,1423}, R_{13}^{3241,3421}, R_{13}^{2431,4231}, R_{13}^{3124,3142}, R_{13}^{2413,4213}, R_{14}^{1423,1432}, R_{14}^{1234,1324}, \\
& R_{14}^{4231,4321}, R_{14}^{2341,3241}, R_{14}^{4123,4132}, R_{14}^{2314,3214}, R_{23}^{2314,2341}, R_{23}^{2143,2413}, R_{23}^{3142,3412}, \\
& R_{23}^{1432,4132}, R_{23}^{3214,3241}, R_{23}^{1423,4123}, R_{24}^{2413,2431}, R_{24}^{2134,2314}, R_{24}^{4132,4312}, R_{24}^{1342,3142}, \\
& R_{24}^{4213,4231}, R_{24}^{1324,3124}, R_{34}^{3412,3421}, R_{34}^{3124,3214}, R_{34}^{4123,4213}, R_{34}^{1243,2143}, R_{34}^{4312,4321}, \\
& R_{34}^{1234,2134}
\end{aligned}
$$

and edges

$$
\begin{gathered}
f_{1234}: Q^{1234} \rightarrow P, f_{1243}: Q^{1243} \rightarrow P, f_{1324}: Q^{1324} \rightarrow P, f_{1342}: Q^{1342} \rightarrow P, \\
f_{1423}: Q^{1423} \rightarrow P, f_{1432}: Q^{1432} \rightarrow P, f_{2134}: Q^{2134} \rightarrow P, f_{2143}: Q^{2143} \rightarrow P, \\
f_{2314}: Q^{2314} \rightarrow P, f_{2341}: Q^{2341} \rightarrow P, f_{2413}: Q^{2413} \rightarrow P, f_{2431}: Q^{2431} \rightarrow P, \\
f_{3124}: Q^{3124} \rightarrow P, f_{3142}: Q^{3142} \rightarrow P, f_{3214}: Q^{3214} \rightarrow P, f_{3241}: Q^{3241} \rightarrow P, \\
f_{3412}: Q^{3412} \rightarrow P, f_{3421}: Q^{3421} \rightarrow P, f_{4123}: Q^{4123} \rightarrow P, f_{4132}: Q^{4132} \rightarrow P, \\
f_{4213}: Q^{4213} \rightarrow P, f_{4231}: Q^{4231} \rightarrow P, f_{4312}: Q^{4312} \rightarrow P, f_{4321}: Q^{4321} \rightarrow P, \\
g_{12}^{1234,1243}: R_{12}^{1234,1243} \rightarrow Q^{1234}, h_{12}^{1234,1243}: R_{12}^{1234,1243} \rightarrow Q^{1243}, \\
g_{12}^{1342,1432}: R_{12}^{1342,1432} \rightarrow Q^{1342}, h_{12}^{1342,1432}: R_{12}^{1342,1432} \rightarrow Q^{1432}, \\
g_{12}^{2341,2431}: R_{12}^{2341,2431} \rightarrow Q^{2341}, h_{12}^{2341,2431}: R_{12}^{2341,2431} \rightarrow Q^{2431}, \\
g_{12}^{3421,4321}: R_{12}^{3421,4321} \rightarrow Q^{3421}, h_{12}^{3421,4321}: R_{12}^{3421,4321} \rightarrow Q^{4321}, \\
g_{12}^{2134,2143}: R_{12}^{2134,2143} \rightarrow Q^{2134}, h_{12}^{2134,2143}: R_{12}^{2134,2143} \rightarrow Q^{2143}, \\
g_{12}^{3412,4312}: R_{12}^{3412,4312} \rightarrow Q^{3412}, h_{12}^{3412,4312}: R_{12}^{3412,4312} \rightarrow Q^{4312}, \\
g_{13}^{1324,1342}: R_{13}^{1324,1342} \rightarrow Q^{1324}, h_{13}^{1324,1342}: R_{13}^{1324,1342} \rightarrow Q^{1342},
\end{gathered}
$$




$$
\begin{aligned}
& g_{13}^{1243,1423}: R_{13}^{1243,1423} \rightarrow Q^{1243}, h_{13}^{1243,1423}: R_{13}^{1243,1423} \rightarrow Q^{1423}, \\
& g_{13}^{3241,3421}: R_{13}^{3241,3421} \rightarrow Q^{3241}, h_{13}^{3241,3421}: R_{13}^{3241,3421} \rightarrow Q^{3421} \text {, } \\
& g_{13}^{2431,4231}: R_{13}^{2431,4231} \rightarrow Q^{2431}, h_{13}^{2431,4231}: R_{13}^{2431,4231} \rightarrow Q^{4231} \text {, } \\
& g_{13}^{3124,3142}: R_{13}^{3124,3142} \rightarrow Q^{3124}, h_{13}^{3124,3142}: R_{13}^{3124,3142} \rightarrow Q^{3142} \text {, } \\
& g_{13}^{2413,4213}: R_{13}^{2413,4213} \rightarrow Q^{2413}, h_{13}^{2413,4213}: R_{13}^{2413,4213} \rightarrow Q^{4213} \text {, } \\
& g_{14}^{1423,1432}: R_{14}^{1423,1432} \rightarrow Q^{1423}, h_{14}^{1423,1432}: R_{14}^{1423,1432} \rightarrow Q^{1432}, \\
& g_{14}^{1234,1324}: R_{14}^{1234,1324} \rightarrow Q^{1234}, h_{14}^{1234,1324}: R_{14}^{1234,1324} \rightarrow Q^{1324} \text {, } \\
& g_{14}^{4231,4321}: R_{14}^{4231,4321} \rightarrow Q^{4231}, h_{14}^{4231,4321}: R_{14}^{4231,4321} \rightarrow Q^{4321} \text {, } \\
& g_{14}^{2341,3241}: R_{14}^{2341,3241} \rightarrow Q^{2341}, h_{14}^{2341,3241}: R_{14}^{2341,3241} \rightarrow Q^{3241} \text {, } \\
& g_{14}^{4123,4132}: R_{14}^{4123,4132} \rightarrow Q^{4123}, h_{14}^{4123,4132}: R_{14}^{4123,4132} \rightarrow Q^{4132} \text {, } \\
& g_{14}^{2314,3214}: R_{14}^{2314,3214} \rightarrow Q^{2314}, h_{14}^{2314,3214}: R_{14}^{2314,3214} \rightarrow Q^{3214} \text {, } \\
& g_{23}^{2314,2341}: R_{23}^{2314,2341} \rightarrow Q^{2314}, h_{23}^{2314,2341}: R_{23}^{2314,2341} \rightarrow Q^{2341} \text {, } \\
& g_{23}^{2143,2413}: R_{23}^{2143,2413} \rightarrow Q^{2143}, h_{23}^{2143,2413}: R_{23}^{2143,2413} \rightarrow Q^{2413} \text {, } \\
& g_{23}^{3142,3412}: R_{23}^{3142,3412} \rightarrow Q^{3142}, h_{23}^{3142,3412}: R_{23}^{3142,3412} \rightarrow Q^{3412}, \\
& g_{23}^{1432,4132}: R_{23}^{1432,4132} \rightarrow Q^{1432}, h_{23}^{1432,4132}: R_{23}^{1432,4132} \rightarrow Q^{4132} \text {, } \\
& g_{23}^{3214,3241}: R_{23}^{3214,3241} \rightarrow Q^{3214}, h_{23}^{3214,3241}: R_{23}^{3214,3241} \rightarrow Q^{3241} \text {, } \\
& g_{23}^{1423,4123}: R_{23}^{1423,4123} \rightarrow Q^{1423}, h_{23}^{1423,4123}: R_{23}^{1423,4123} \rightarrow Q^{4123} \text {, } \\
& g_{24}^{2413,2431}: R_{24}^{2413,2431} \rightarrow Q^{2413}, h_{24}^{2413,2431}: R_{24}^{2413,2431} \rightarrow Q^{2431}, \\
& g_{24}^{2134,2314}: R_{24}^{2134,2314} \rightarrow Q^{2134}, h_{24}^{2134,2314}: R_{24}^{2134,2314} \rightarrow Q^{2314} \text {, } \\
& g_{24}^{4132,4312}: R_{24}^{4132,4312} \rightarrow Q^{4132}, h_{24}^{4132,4312}: R_{24}^{4132,4312} \rightarrow Q^{4312} \text {, } \\
& g_{24}^{1342,3142}: R_{24}^{1342,3142} \rightarrow Q^{1342}, h_{24}^{1342,3142}: R_{24}^{1342,3142} \rightarrow Q^{3142} \text {, } \\
& g_{24}^{4213,4231}: R_{24}^{4213,4231} \rightarrow Q^{4213}, h_{24}^{4213,4231}: R_{24}^{4213,4231} \rightarrow Q^{4231} \text {, } \\
& g_{24}^{1324,3124}: R_{24}^{1324,3124} \rightarrow Q^{1324}, h_{24}^{1324,3124}: R_{24}^{1324,3124} \rightarrow Q^{3124} \text {, } \\
& g_{34}^{3412,3421}: R_{34}^{3412,3421} \rightarrow Q^{3412}, h_{34}^{3412,3421}: R_{34}^{3412,3421} \rightarrow Q^{3421}, \\
& g_{34}^{3124,3214}: R_{34}^{3124,3214} \rightarrow Q^{3124}, h_{34}^{3124,3214}: R_{34}^{3124,3214} \rightarrow Q^{3214} \text {, } \\
& g_{34}^{4123,4213}: R_{34}^{4123,4213} \rightarrow Q^{4123}, h_{34}^{4123,4213}: R_{34}^{4123,4213} \rightarrow Q^{4213} \text {, } \\
& g_{34}^{1243,2143}: R_{34}^{1243,2143} \rightarrow Q^{1243}, h_{34}^{1243,2143}: R_{34}^{1243,2143} \rightarrow Q^{2143} \text {, } \\
& g_{34}^{4312,4321}: R_{34}^{4312,4321} \rightarrow Q^{4312}, h_{34}^{4312,4321}: R_{34}^{4312,4321} \rightarrow Q^{4321} \text {, } \\
& g_{34}^{1234,2134}: R_{34}^{1234,2134} \rightarrow Q^{1234}, h_{34}^{1234,2134}: R_{34}^{1234,2134} \rightarrow Q^{2134}
\end{aligned}
$$


with $P$ being labelled

$$
D^{53}\left\{\begin{array}{c}
(1,5),(2,5),(1,6),(3,6),(1,7),(4,7),(2,8),(3,8),(2,9),(4,9), \\
(3,10),(4,10),(5,6),(5,7),(5,8),(5,9),(6,7),(6,8),(6,10),(7,9), \\
(7,10),(8,9),(8,10),(9,10),\left(1, i_{11,15}\right),\left(2, i_{11,15}\right),\left(3, i_{11,15}\right), \\
\left(5, i_{11,15}\right),\left(6, i_{11,15}\right),\left(7, i_{11,15}\right),\left(8, i_{11,15}\right),\left(9, i_{11,15}\right),\left(10, i_{11,15}\right), \\
\left(1, i_{16,20}\right),\left(2, i_{16,20}\right),\left(4, i_{16,20}\right),\left(5, i_{16,20}\right),\left(6, i_{16,20}\right),\left(7, i_{16,20}\right), \\
\left(8, i_{16,20}\right),\left(9, i_{16,20}\right),\left(10, i_{16,20}\right),\left(1, i_{21,25}\right)\left(3, i_{21,25}\right),\left(4, i_{21,25}\right), \\
\left(5, i_{21,25}\right),\left(6, i_{21,25}\right),\left(7, i_{21,25}\right),\left(8, i_{21,25}\right),\left(9, i_{21,25}\right),\left(10, i_{21,25}\right), \\
\left(2, i_{26,30}\right),\left(3, i_{26,30}\right),\left(4, i_{26,30}\right),\left(5, i_{26,30}\right),\left(6, i_{26,30}\right),\left(7, i_{26,30}\right), \\
\left(8, i_{26,30}\right),\left(9, i_{26,30}\right),\left(10, i_{26,30}\right),\left(i_{11,15}, i_{11,15}^{\prime}\right),\left(i_{11,15}, i_{16,20}\right), \\
\left(i_{11,15}, i_{21,25}\right),\left(i_{11,15}, i_{26,30}\right),\left(i_{16,20}, i_{16,20}^{\prime}\right),\left(i_{16,20}, i_{21,25}\right), \\
\left(i_{16,20}, i_{26,30}\right),\left(i_{21,25}, i_{21,25}^{\prime}\right),\left(i_{21,25}, i_{26,30}\right),\left(i_{26,30}, i_{26,30}^{\prime}\right), \\
\left(1, i_{31,53}\right),\left(2, i_{31,53}\right),\left(3, i_{31,53}\right),\left(4, i_{31,53}\right),\left(5, i_{31,53}\right),\left(6, i_{31,53}\right), \\
\left(7, i_{31,53}\right),\left(8, i_{31,53}\right),\left(9, i_{31,53}\right),\left(10, i_{31,53}\right),\left(i_{11,15}, i_{31,53}\right), \\
\left(i_{16,20}, i_{31,53}\right),\left(i_{21,25}, i_{31,53}\right),\left(i_{26,30}, i_{31,53}\right),\left(i_{31,53}, i_{31,53}^{\prime}\right) \\
1 i_{11,15}, i_{11,15}^{\prime}, i_{16,20}, i_{16,20}^{\prime}, i_{21,25}, i_{21,25}^{\prime}, i_{26,30}, i_{26,30}^{\prime}, i_{31,53}, i_{31,53}^{\prime} \in \mathbb{N}, \\
1 \leq i_{11,15} \leq 15,11 \leq i_{11,15}^{\prime} \leq 15,16 \leq i_{16,20} \leq 20, \\
16 \leq i_{16,20}^{\prime} \leq 20,21 \leq i_{21,25} \leq 25,21 \leq i_{21,25}^{\prime} \leq 25, \\
26 \leq i_{26,30} \leq 30,26 \leq i_{26,30}^{\prime} \leq 30,31 \leq i_{31,53} \leq 53, \\
31 \leq i_{31,53}^{\prime} \leq 53
\end{array}\right\}
$$

all of

$$
\begin{aligned}
& Q^{1234}, Q^{1243}, Q^{1324}, Q^{1342}, Q^{1423}, Q^{1432}, Q^{2134}, Q^{2143}, Q^{2314}, Q^{2341}, Q^{2413}, Q^{2431}, \\
& Q^{3124}, Q^{3142}, Q^{3214}, Q^{3241}, Q^{3412}, Q^{3421}, Q^{4123}, Q^{4132}, Q^{4213}, Q^{4231}, Q^{4312}, Q^{4321}
\end{aligned}
$$

being laballed $D^{4}$, all of

$$
R_{12}^{1234,1243}, R_{12}^{1342,1432}, R_{12}^{2341,2431}, R_{12}^{3421,4321}, R_{12}^{2134,2143}, R_{12}^{3412,4312}
$$

being labelled $D^{4}\{(3,4)\}$, all of

$$
R_{13}^{1324,1342}, R_{13}^{1243,1423}, R_{13}^{3241,3421}, R_{13}^{2431,4231}, R_{13}^{3124,3142}, R_{13}^{2413,4213}
$$

being labelled $D^{4}\{(2,4)\}$, all of

$$
R_{14}^{1423,1432}, R_{14}^{1234,1324}, R_{14}^{4231,4321}, R_{14}^{2341,3241}, R_{14}^{4123,4132}, R_{14}^{2314,3214}
$$

being labelled $D^{4}\{(2,3)\}$, all of

$$
R_{23}^{2314,2341}, R_{23}^{2143,2413}, R_{23}^{3142,3412}, R_{23}^{1432,4132}, R_{23}^{3214,3241}, R_{23}^{1423,4123}
$$

being labelled $D^{4}\{(1,4)\}$, all of

$$
R_{24}^{2413,2431}, R_{24}^{2134,2314}, R_{24}^{4132,4312}, R_{24}^{1342,3142}, R_{24}^{4213,4231}, R_{24}^{1324,3124}
$$

being labelled $D^{4}\{(1,3)\}$

$$
R_{34}^{3412,3421}, R_{34}^{3124,3214}, R_{34}^{4123,4213}, R_{34}^{1243,2143}, R_{34}^{4312,4321}, R_{34}^{1234,2134}
$$

being labelled $D^{4}\{(1,2)\}$, the edges

$f_{1234}, f_{1243}, f_{1324}, f_{1342}, f_{1423}, f_{1432}, f_{2134}, f_{2143}, f_{2314}, f_{2341}, f_{2413}, f_{2431}$,

$f_{3124}, f_{3142}, f_{3214}, f_{3241}, f_{3412}, f_{3421}, f_{4123}, f_{4132}, f_{4213}, f_{4231}, f_{4312}, f_{4321}$ 
standing for mappings

$$
\begin{aligned}
& f_{1234}\left(d_{1}, d_{2}, d_{3}, d_{4}\right) \\
& =\left(d_{1}, d_{2}, d_{3}, d_{4},{ }_{5}, \ldots,{ }_{53}^{0}\right), \\
& f_{1243}\left(d_{1}, d_{2}, d_{3}, d_{4}\right) \\
& =\left(\begin{array}{c}
d_{1}, d_{2}, d_{3}, d_{4}, 0, \ldots, 0,{ }_{5}^{0}, d_{3} d_{4}, \underset{11}{0}, \ldots, \underset{20}{0}, d_{1} d_{3} d_{4}, \underset{22}{0}, \ldots, \underset{25}{0}, d_{2} d_{3} d_{4}, 0, \ldots,{ }_{27}^{0}, \\
d_{1} d_{2} d_{3} d_{4},{ }_{32}^{0}, \ldots,{ }_{53}^{0}
\end{array}\right),
\end{aligned}
$$

$f_{1324}\left(d_{1}, d_{2}, d_{3}, d_{4}\right)$

$$
=\left(\begin{array}{c}
d_{1}, d_{2}, d_{3}, d_{4}, \underset{5}{0}, \ldots, \underset{7}{0}, d_{2} d_{3}, \underset{9}{0}, \underset{10}{0}, d_{1} d_{2} d_{3}, \underset{12}{0}, \ldots, \underset{26}{0}, d_{2} d_{3} d_{4}, \underset{28}{0}, \ldots, \underset{31}{0} \\
d_{1} d_{2} d_{3} d_{4}, \underset{33}{0}, \ldots,{ }_{53}^{0}
\end{array}\right)
$$

$$
\begin{aligned}
& f_{1342}\left(d_{1}, d_{2}, d_{3}, d_{4}\right) \\
& =\left(\begin{array}{c}
d_{1}, d_{2}, d_{3}, d_{4}, \underset{5}{0}, \ldots, \underset{7}{0}, d_{2} d_{3}, d_{2} d_{4}, \underset{10}{0}, d_{1} d_{2} d_{3}, \underset{12}{0}, \ldots, \underset{15}{0}, d_{1} d_{2} d_{4}, \underset{17}{0}, \ldots, \underset{27}{0} \\
d_{2} d_{3} d_{4}, \underset{29}{0}, \ldots, \underset{32}{0}, d_{1} d_{2} d_{3} d_{4}, \underset{34}{0}, \ldots, \underset{53}{0}
\end{array}\right),
\end{aligned}
$$

$$
\begin{aligned}
& f_{1423}\left(d_{1}, d_{2}, d_{3}, d_{4}\right) \\
& =\left(\begin{array}{c}
d_{1}, d_{2}, d_{3}, d_{4}, \underset{5}{0}, \ldots, \underset{8}{0}, d_{2} d_{4}, d_{3} d_{4}, \underset{11}{0}, \ldots, \underset{15}{0}, d_{1} d_{2} d_{4}, \underset{17}{0}, \ldots, \underset{20}{0}, d_{1} d_{3} d_{4}, \\
\underset{22}{0}, \ldots, \underset{28}{0}, d_{2} d_{3} d_{4}, \underset{30}{0}, \ldots, \underset{33}{0}, d_{1} d_{2} d_{3} d_{4}, \underset{35}{0}, \ldots, \underset{53}{0}
\end{array}\right),
\end{aligned}
$$

$$
\begin{aligned}
& f_{1432}\left(d_{1}, d_{2}, d_{3}, d_{4}\right) \\
& =\left(\begin{array}{c}
d_{1}, d_{2}, d_{3}, d_{4}, \underset{5}{0}, \ldots, \underset{7}{0}, d_{2} d_{3}, d_{2} d_{4}, d_{3} d_{4}, d_{1} d_{2} d_{3}, \underset{12}{0}, \ldots, \underset{15}{0}, d_{1} d_{2} d_{4}, \underset{16}{0}, \ldots, \underset{20}{0}, \\
d_{1} d_{3} d_{4}, \underset{22}{0}, \ldots, \underset{29}{0}, d_{2} d_{3} d_{4}, \underset{31}{0}, \ldots, \underset{34}{0}, d_{1} d_{2} d_{3} d_{4}, \underset{36}{0}, \ldots, \underset{53}{0}
\end{array}\right),
\end{aligned}
$$

$$
\begin{aligned}
& f_{2134}\left(d_{1}, d_{2}, d_{3}, d_{4}\right) \\
& =\left(\begin{array}{c}
d_{1}, d_{2}, d_{3}, d_{4}, d_{1} d_{2}, \underset{6}{0}, \ldots, \underset{11}{0}, d_{1} d_{2} d_{3}, \underset{13}{0}, \ldots, \underset{16}{0}, d_{1} d_{2} d_{4}, \\
\underset{18}{0}, \ldots, \underset{35}{0}, d_{1} d_{2} d_{3} d_{4}, \underset{37}{0}, \ldots, \underset{53}{0}
\end{array}\right),
\end{aligned}
$$

$$
\begin{aligned}
& f_{2143}\left(d_{1}, d_{2}, d_{3}, d_{4}\right) \\
& =\left(\begin{array}{c}
d_{1}, d_{2}, d_{3}, d_{4}, d_{1} d_{2}, \underset{6}{0}, \ldots, \underset{9}{0}, d_{3} d_{4}, \underset{11}{0}, d_{1} d_{2} d_{3}, \underset{13}{0}, \ldots, \underset{16}{0}, d_{1} d_{2} d_{4}, \underset{18}{0}, \ldots, \underset{20}{0}, \\
d_{1} d_{3} d_{4}, \underset{22}{0}, \ldots, \underset{25}{0}, d_{2} d_{3} d_{4}, \underset{27}{0}, \ldots, \underset{36}{0}, d_{1} d_{2} d_{3} d_{4}, \underset{38}{0}, \ldots, \underset{53}{0}
\end{array}\right),
\end{aligned}
$$

$$
\begin{aligned}
& f_{2314}\left(d_{1}, d_{2}, d_{3}, d_{4}\right) \\
& =\left(\begin{array}{c}
d_{1}, d_{2}, d_{3}, d_{4}, d_{1} d_{2}, d_{1} d_{3}, \underset{7}{0}, \ldots, \underset{12}{0}, d_{1} d_{2} d_{3}, \underset{14}{0}, \ldots, \underset{16}{0}, d_{1} d_{2} d_{4}, \underset{18}{0}, \ldots, \underset{21}{0}, d_{1} d_{3} d_{4}, \\
0, \ldots, \underset{37}{0}, d_{1} d_{2} d_{3} d_{4}, \underset{39}{0}, \ldots, \underset{53}{0}
\end{array}\right),
\end{aligned}
$$

$f_{2341}\left(d_{1}, d_{2}, d_{3}, d_{4}\right)$ 
$=\left(\begin{array}{c}d_{1}, d_{2}, d_{3}, d_{4}, d_{1} d_{2}, d_{1} d_{3}, d_{1} d_{4}, \underset{8}{0}, \ldots, \underset{12}{0}, d_{1} d_{2} d_{3}, \underset{14}{0}, \ldots, \underset{17}{0}, d_{1} d_{2} d_{4}, \underset{19}{0}, \ldots, \underset{22}{0}, \\ d_{1} d_{3} d_{4}, \underset{24}{0}, \ldots, \underset{38}{0}, d_{1} d_{2} d_{3} d_{4}, \underset{40}{0}, \ldots,{ }_{53}^{0}\end{array}\right)$,

$f_{2413}\left(d_{1}, d_{2}, d_{3}, d_{4}\right)$

$=\left(\begin{array}{c}d_{1}, d_{2}, d_{3}, d_{4}, d_{1} d_{2}, \underset{6}{0}, d_{1} d_{4}, \underset{8}{0}, \underset{9}{0}, d_{3} d_{4}, \underset{11}{0}, d_{1} d_{2} d_{3}, \underset{13}{0}, \ldots, \underset{17}{0}, d_{1} d_{2} d_{4}, \underset{19}{0}, \ldots, \underset{23}{0}, \\ d_{1} d_{3} d_{4}, \underset{25}{0}, d_{2} d_{3} d_{4}, \underset{27}{0}, \ldots,{ }_{39}^{0}, d_{1} d_{2} d_{3} d_{4}, \underset{41}{0}, \ldots,{ }_{53}^{0}\end{array}\right)$,

$f_{2431}\left(d_{1}, d_{2}, d_{3}, d_{4}\right)$

$=\left(\begin{array}{c}d_{1}, d_{2}, d_{3}, d_{4}, d_{1} d_{2}, d_{1} d_{3}, d_{1} d_{4}, \underset{8}{0}, \underset{9}{0}, d_{3} d_{4}, \underset{11}{0}, \underset{12}{0}, d_{1} d_{2} d_{3}, \underset{14}{0}, \ldots, \underset{17}{0}, d_{1} d_{2} d_{4}, \\ 0, \ldots, \underset{24}{0}, d_{1} d_{3} d_{4}, d_{2} d_{3} d_{4}, \underset{27}{0}, \ldots, \underset{40}{0}, d_{1} d_{2} d_{3} d_{4}, \underset{42}{0}, \ldots,{ }_{53}^{0}\end{array}\right)$,

$f_{3124}\left(d_{1}, d_{2}, d_{3}, d_{4}\right)$

$=\left(\begin{array}{c}d_{1}, d_{2}, d_{3}, d_{4}, \underset{5}{0}, d_{1} d_{3}, \underset{7}{0}, d_{2} d_{3}, \underset{9}{0}, \ldots, \underset{13}{0}, d_{1} d_{2} d_{3}, \underset{15}{0}, \ldots, \underset{21}{0}, d_{1} d_{3} d_{4}, \underset{23}{0}, \ldots, \underset{26}{0}, \\ d_{2} d_{3} d_{4}, \underset{28}{0}, \ldots, \underset{41}{0}, d_{1} d_{2} d_{3} d_{4}, \underset{43}{0}, \ldots, \underset{53}{0}\end{array}\right)$,

$f_{3142}\left(d_{1}, d_{2}, d_{3}, d_{4}\right)$

$=\left(\begin{array}{c}d_{1}, d_{2}, d_{3}, d_{4}, \underset{5}{0}, d_{1} d_{3}, \underset{7}{0}, d_{2} d_{3}, d_{2} d_{4}, \underset{10}{0}, \ldots, \underset{13}{0}, d_{1} d_{2} d_{3}, \underset{15}{0}, d_{1} d_{2} d_{4}, \underset{17}{0}, \ldots, \underset{21}{0}, \\ d_{1} d_{3} d_{4}, \underset{23}{0}, \ldots, \underset{27}{0}, d_{2} d_{3} d_{4}, \underset{29}{0}, \ldots, \underset{42}{0}, d_{1} d_{2} d_{3} d_{4}, \underset{44}{0}, \ldots, \underset{53}{0}\end{array}\right)$,

$f_{3214}\left(d_{1}, d_{2}, d_{3}, d_{4}\right)$

$=\left(\begin{array}{c}d_{1}, d_{2}, d_{3}, d_{4}, d_{1} d_{2}, d_{1} d_{3}, \underset{7}{0}, d_{2} d_{3}, \underset{9}{0}, \ldots, \underset{14}{0}, d_{1} d_{2} d_{3}, \underset{16}{0}, d_{1} d_{2} d_{4}, \underset{18}{0}, \ldots, \underset{21}{0}, \\ d_{1} d_{3} d_{4}, \underset{23}{0}, \ldots, \underset{26}{0}, d_{2} d_{3} d_{4}, \underset{28}{0}, \ldots, \underset{43}{0}, d_{1} d_{2} d_{3} d_{4}, \underset{45}{0}, \ldots, \underset{53}{0}\end{array}\right)$,

$f_{3241}\left(d_{1}, d_{2}, d_{3}, d_{4}\right)$

$=\left(\begin{array}{c}d_{1}, d_{2}, d_{3}, d_{4}, d_{1} d_{2}, d_{1} d_{3}, d_{1} d_{4}, d_{2} d_{3}, \underset{9}{0}, \ldots, \underset{14}{0}, d_{1} d_{2} d_{3}, \underset{16}{0} \underset{17}{0}, d_{1} d_{2} d_{4}, \underset{19}{0}, \ldots, \underset{22}{0}, \\ d_{1} d_{3} d_{4}, \underset{24}{0}, \ldots, \underset{26}{0}, d_{2} d_{3} d_{4}, \underset{28}{0}, \ldots, \underset{44}{0}, d_{1} d_{2} d_{3} d_{4}, \underset{46}{0}, \ldots, \underset{53}{0}\end{array}\right)$,

$f_{3412}\left(d_{1}, d_{2}, d_{3}, d_{4}\right)$

$=\left(\begin{array}{c}d_{1}, d_{2}, d_{3}, d_{4}, \underset{5}{0}, d_{1} d_{3}, d_{1} d_{4}, d_{2} d_{3}, d_{2} d_{4}, \underset{10}{0}, \ldots, \underset{13}{0}, d_{1} d_{2} d_{3}, \underset{15}{0}, \ldots, \underset{18}{0}, d_{1} d_{2} d_{4}, \\ 0, \ldots, \underset{22}{0}, d_{1} d_{3} d_{4}, \underset{24}{0}, \ldots, \underset{27}{0}, d_{2} d_{3} d_{4}, \underset{29}{0}, \ldots, \underset{45}{0}, d_{1} d_{2} d_{3} d_{4}, \underset{47}{0}, \ldots, \underset{53}{0}\end{array}\right)$,

$f_{3421}\left(d_{1}, d_{2}, d_{3}, d_{4}\right)$

$=\left(\begin{array}{c}d_{1}, d_{2}, d_{3}, d_{4}, d_{1} d_{2}, d_{1} d_{3}, d_{1} d_{4}, d_{2} d_{3}, d_{2} d_{4}, \underset{10}{0}, \ldots, \underset{14}{0}, d_{1} d_{2} d_{3}, \underset{16}{0}, \ldots, \underset{19}{0}, d_{1} d_{2} d_{4}, \\ \underset{21}{0}, \underset{22}{0}, d_{1} d_{3} d_{4}, \underset{24}{0}, \ldots, \underset{27}{0}, d_{2} d_{3} d_{4}, \underset{29}{0}, \ldots, \underset{46}{0}, d_{1} d_{2} d_{3} d_{4}, \underset{48}{0}, \ldots, \underset{53}{0}\end{array}\right)$,

$f_{4123}\left(d_{1}, d_{2}, d_{3}, d_{4}\right)$

$=\left(\begin{array}{c}d_{1}, d_{2}, d_{3}, d_{4}, \underset{5}{0} \underset{6}{0}, d_{1} d_{4}, \underset{7}{0}, \underset{7}{0}, d_{2} d_{4}, d_{3} d_{4}, \underset{11}{0}, \ldots, \underset{18}{0}, d_{1} d_{2} d_{4}, \underset{20}{0}, \ldots, \underset{23}{0}, d_{1} d_{3} d_{4}, \\ 0, \ldots, \underset{28}{0}, d_{2} d_{3} d_{4}, \underset{30}{0}, \ldots, \underset{47}{0}, d_{1} d_{2} d_{3} d_{4}, \underset{49}{0}, \ldots, \underset{53}{0}\end{array}\right)$, 


$$
\begin{aligned}
& f_{4132}\left(d_{1}, d_{2}, d_{3}, d_{4}\right) \\
& =\left(\begin{array}{c}
d_{1}, d_{2}, d_{3}, d_{4}, \underset{5}{0}, \underset{6}{0}, d_{1} d_{4}, d_{2} d_{3}, d_{2} d_{4}, d_{3} d_{4}, d_{1} d_{2} d_{3}, \underset{12}{0}, \ldots, \underset{18}{0}, d_{1} d_{2} d_{4}, \underset{20}{0}, \ldots, \underset{23}{0}, \\
d_{1} d_{3} d_{4}, \underset{25}{0}, \ldots, \underset{29}{0}, d_{2} d_{3} d_{4}, \underset{31}{0}, \ldots, \underset{48}{0}, d_{1} d_{2} d_{3} d_{4}, \underset{50}{0}, \ldots, \underset{53}{0}
\end{array}\right), \\
& f_{4213}\left(d_{1}, d_{2}, d_{3}, d_{4}\right) \\
& =\left(\begin{array}{c}
d_{1}, d_{2}, d_{3}, d_{4}, d_{1} d_{2}, \underset{6}{0}, d_{1} d_{4}, \underset{8}{0}, d_{2} d_{4}, d_{3} d_{4}, \underset{11}{0}, d_{1} d_{2} d_{3}, \underset{13}{0}, \ldots, \underset{19}{0}, d_{1} d_{2} d_{4}, \\
0, \ldots, \underset{23}{0}, d_{1} d_{3} d_{4}, \underset{25}{0}, \ldots, \underset{28}{0}, d_{2} d_{3} d_{4}, \underset{30}{0}, \ldots, \underset{49}{0}, d_{1} d_{2} d_{3} d_{4}, \underset{51}{0}, \ldots, \underset{53}{0}
\end{array}\right), \\
& f_{4231}\left(d_{1}, d_{2}, d_{3}, d_{4}\right)
\end{aligned}
$$

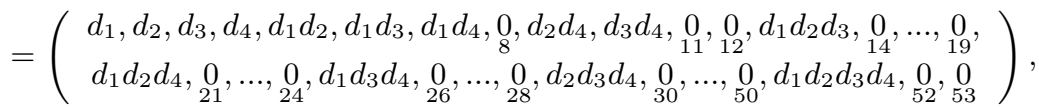

$$
\begin{aligned}
& f_{4312}\left(d_{1}, d_{2}, d_{3}, d_{4}\right)
\end{aligned}
$$

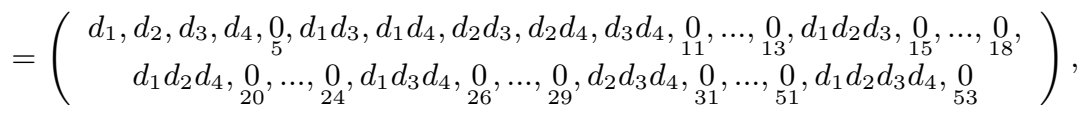

$$
\begin{aligned}
& f_{4321}\left(d_{1}, d_{2}, d_{3}, d_{4}\right)
\end{aligned}
$$

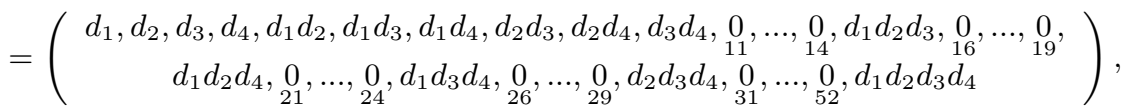

respectively, and all the other edges standing for the identity mappings (e.g., both $g_{12}^{1234,1243}$ and $h_{12}^{1234,1243}$ standing for the mapping $\left(d_{1}, d_{2}, d_{3}, d_{4}\right) \in D^{4}\{(3,4)\} \mapsto$ $\left(d_{1}, d_{2}, d_{3}, d_{4}\right) \in D^{4}$ while both $g_{13}^{1324,1342}$ and $h_{13}^{1324,1342}$ standing for the mapping $\left.\left(d_{1}, d_{2}, d_{3}, d_{4}\right) \in D^{4}\{(2,4)\} \mapsto\left(d_{1}, d_{2}, d_{3}, d_{4}\right) \in D^{4}\right)$ is a quasi-colimit diagram.

Proof. Let $\theta^{1234}: Q^{1234} \rightarrow \mathbb{R}, \theta^{1243}: Q^{1243} \rightarrow \mathbb{R}, \theta^{1324}: Q^{1324} \rightarrow \mathbb{R}, \theta^{1342}:$ $Q^{1342} \rightarrow \mathbb{R}, \theta^{1423}: Q^{1423} \rightarrow \mathbb{R}, \theta^{1432}: Q^{1432} \rightarrow \mathbb{R}, \theta^{2134}: Q^{2134} \rightarrow \mathbb{R}, \theta^{2143}:$ $Q^{2143} \rightarrow \mathbb{R}, \theta^{2314}: Q^{2314} \rightarrow \mathbb{R}, \theta^{2341}: Q^{2341} \rightarrow \mathbb{R}, \theta^{2413}: Q^{2413} \rightarrow \mathbb{R}, \theta^{2431}:$ $Q^{2431} \rightarrow \mathbb{R}, \theta^{3124}: Q^{3124} \rightarrow \mathbb{R}, \theta^{3142}: Q^{3142} \rightarrow \mathbb{R}, \theta^{3214}: Q^{3214} \rightarrow \mathbb{R}, \theta^{3241}:$ $Q^{3241} \rightarrow \mathbb{R}, \theta^{3412}: Q^{3412} \rightarrow \mathbb{R}, \theta^{3421}: Q^{3421} \rightarrow \mathbb{R}, \theta^{4123}: Q^{4123} \rightarrow \mathbb{R}, \theta^{4132}:$ $Q^{4132} \rightarrow \mathbb{R}, \theta^{4213}: Q^{4213} \rightarrow \mathbb{R}, \theta^{4231}: Q^{4231} \rightarrow \mathbb{R}, \theta^{4312}: Q^{4312} \rightarrow \mathbb{R}$ and $\theta^{4321}: Q^{4321} \rightarrow \mathbb{R}$ be mappings, which are to be of the following forms by dint of the general Kock-Lawvere axiom (cf. $§ 2.1 .3$ of [4]):

$$
\begin{aligned}
& \theta^{1234}\left(d_{1}, d_{2}, d_{3}, d_{4}\right) \\
& =a^{1234}+a_{1}^{1234} d_{1}+a_{2}^{1234} d_{2}+a_{3}^{1234} d_{3}+a_{4}^{1234} d_{1}+a_{12}^{1234} d_{1} d_{2}+a_{13}^{1234} d_{1} d_{3}+ \\
& a_{14}^{1234} d_{1} d_{4}+a_{23}^{1234} d_{2} d_{3}+a_{24}^{1234} d_{2} d_{4}+a_{34}^{1234} d_{3} d_{4}+a_{123}^{1234} d_{1} d_{2} d_{3}+a_{124}^{1234} d_{1} d_{2} d_{4}+ \\
& a_{134}^{1234} d_{1} d_{3} d_{4}+a_{234}^{1234} d_{2} d_{3} d_{4}+a_{1234}^{1234} d_{1} d_{2} d_{3} d_{4}, \\
& \theta^{1243}\left(d_{1}, d_{2}, d_{3}, d_{4}\right) \\
& =a^{1243}+a_{1}^{1243} d_{1}+a_{2}^{1243} d_{2}+a_{3}^{1243} d_{3}+a_{4}^{1243} d_{1}+a_{12}^{1243} d_{1} d_{2}+a_{13}^{1243} d_{1} d_{3}+
\end{aligned}
$$




$$
\begin{aligned}
& a_{14}^{1243} d_{1} d_{4}+a_{23}^{1243} d_{2} d_{3}+a_{24}^{1243} d_{2} d_{4}+a_{34}^{1243} d_{3} d_{4}+a_{123}^{1243} d_{1} d_{2} d_{3}+a_{124}^{1243} d_{1} d_{2} d_{4}+ \\
& a_{134}^{1243} d_{1} d_{3} d_{4}+a_{234}^{1243} d_{2} d_{3} d_{4}+a_{1234}^{1243} d_{1} d_{2} d_{3} d_{4}, \\
& \theta^{1324}\left(d_{1}, d_{2}, d_{3}, d_{4}\right) \\
& =a^{1324}+a_{1}^{1324} d_{1}+a_{2}^{1324} d_{2}+a_{3}^{1324} d_{3}+a_{4}^{1324} d_{1}+a_{12}^{1324} d_{1} d_{2}+a_{13}^{1324} d_{1} d_{3}+ \\
& a_{14}^{1324} d_{1} d_{4}+a_{23}^{1324} d_{2} d_{3}+a_{24}^{1324} d_{2} d_{4}+a_{34}^{1324} d_{3} d_{4}+a_{123}^{1324} d_{1} d_{2} d_{3}+a_{124}^{1324} d_{1} d_{2} d_{4}+ \\
& a_{134}^{1324} d_{1} d_{3} d_{4}+a_{234}^{1324} d_{2} d_{3} d_{4}+a_{1234}^{1324} d_{1} d_{2} d_{3} d_{4}, \\
& \theta^{1342}\left(d_{1}, d_{2}, d_{3}, d_{4}\right) \\
& =a^{1342}+a_{1}^{1342} d_{1}+a_{2}^{1342} d_{2}+a_{3}^{1342} d_{3}+a_{4}^{1342} d_{1}+a_{12}^{1342} d_{1} d_{2}+a_{13}^{1342} d_{1} d_{3}+ \\
& a_{14}^{1342} d_{1} d_{4}+a_{23}^{1342} d_{2} d_{3}+a_{24}^{1342} d_{2} d_{4}+a_{34}^{1342} d_{3} d_{4}+a_{123}^{1342} d_{1} d_{2} d_{3}+a_{124}^{1342} d_{1} d_{2} d_{4}+ \\
& a_{134}^{1342} d_{1} d_{3} d_{4}+a_{234}^{1342} d_{2} d_{3} d_{4}+a_{1234}^{1342} d_{1} d_{2} d_{3} d_{4}, \\
& \theta^{1423}\left(d_{1}, d_{2}, d_{3}, d_{4}\right) \\
& =a^{1423}+a_{1}^{1423} d_{1}+a_{2}^{1423} d_{2}+a_{3}^{1423} d_{3}+a_{4}^{1423} d_{1}+a_{12}^{1423} d_{1} d_{2}+a_{13}^{1423} d_{1} d_{3}+ \\
& a_{14}^{1423} d_{1} d_{4}+a_{23}^{1423} d_{2} d_{3}+a_{24}^{1423} d_{2} d_{4}+a_{34}^{1423} d_{3} d_{4}+a_{123}^{1423} d_{1} d_{2} d_{3}+a_{124}^{1423} d_{1} d_{2} d_{4}+ \\
& a_{134}^{1423} d_{1} d_{3} d_{4}+a_{234}^{1423} d_{2} d_{3} d_{4}+a_{1234}^{1423} d_{1} d_{2} d_{3} d_{4}, \\
& \theta^{1432}\left(d_{1}, d_{2}, d_{3}, d_{4}\right) \\
& =a^{1432}+a_{1}^{1432} d_{1}+a_{2}^{1432} d_{2}+a_{3}^{1432} d_{3}+a_{4}^{1432} d_{1}+a_{12}^{1432} d_{1} d_{2}+a_{13}^{1432} d_{1} d_{3}+ \\
& a_{14}^{1432} d_{1} d_{4}+a_{23}^{1432} d_{2} d_{3}+a_{24}^{1432} d_{2} d_{4}+a_{34}^{1432} d_{3} d_{4}+a_{123}^{1432} d_{1} d_{2} d_{3}+a_{124}^{1432} d_{1} d_{2} d_{4}+ \\
& a_{134}^{1432} d_{1} d_{3} d_{4}+a_{234}^{1432} d_{2} d_{3} d_{4}+a_{1234}^{1432} d_{1} d_{2} d_{3} d_{4}, \\
& \theta^{2134}\left(d_{1}, d_{2}, d_{3}, d_{4}\right) \\
& =a^{2134}+a_{1}^{2134} d_{1}+a_{2}^{2134} d_{2}+a_{3}^{2134} d_{3}+a_{4}^{2134} d_{1}+a_{12}^{2134} d_{1} d_{2}+a_{13}^{2134} d_{1} d_{3}+ \\
& a_{14}^{2134} d_{1} d_{4}+a_{23}^{2134} d_{2} d_{3}+a_{24}^{2134} d_{2} d_{4}+a_{34}^{2134} d_{3} d_{4}+a_{123}^{2134} d_{1} d_{2} d_{3}+a_{124}^{2134} d_{1} d_{2} d_{4}+ \\
& a_{134}^{2134} d_{1} d_{3} d_{4}+a_{234}^{2134} d_{2} d_{3} d_{4}+a_{1234}^{2134} d_{1} d_{2} d_{3} d_{4} \text {, } \\
& \theta^{2143}\left(d_{1}, d_{2}, d_{3}, d_{4}\right) \\
& =a^{2143}+a_{1}^{2143} d_{1}+a_{2}^{2143} d_{2}+a_{3}^{2143} d_{3}+a_{4}^{2143} d_{1}+a_{12}^{2143} d_{1} d_{2}+a_{13}^{2143} d_{1} d_{3}+ \\
& a_{14}^{2143} d_{1} d_{4}+a_{23}^{2143} d_{2} d_{3}+a_{24}^{2143} d_{2} d_{4}+a_{34}^{2143} d_{3} d_{4}+a_{123}^{2143} d_{1} d_{2} d_{3}+a_{124}^{2143} d_{1} d_{2} d_{4}+ \\
& a_{134}^{2143} d_{1} d_{3} d_{4}+a_{234}^{2143} d_{2} d_{3} d_{4}+a_{1234}^{2143} d_{1} d_{2} d_{3} d_{4}, \\
& \theta^{2314}\left(d_{1}, d_{2}, d_{3}, d_{4}\right) \\
& =a^{2314}+a_{1}^{2314} d_{1}+a_{2}^{2314} d_{2}+a_{3}^{2314} d_{3}+a_{4}^{2314} d_{1}+a_{12}^{2314} d_{1} d_{2}+a_{13}^{2314} d_{1} d_{3}+ \\
& a_{14}^{2314} d_{1} d_{4}+a_{23}^{2314} d_{2} d_{3}+a_{24}^{2314} d_{2} d_{4}+a_{34}^{2314} d_{3} d_{4}+a_{123}^{2314} d_{1} d_{2} d_{3}+a_{124}^{2314} d_{1} d_{2} d_{4}+ \\
& a_{134}^{2314} d_{1} d_{3} d_{4}+a_{234}^{2314} d_{2} d_{3} d_{4}+a_{1234}^{2314} d_{1} d_{2} d_{3} d_{4} \text {, } \\
& \theta^{2341}\left(d_{1}, d_{2}, d_{3}, d_{4}\right)
\end{aligned}
$$




$$
\begin{aligned}
& =a^{2341}+a_{1}^{2341} d_{1}+a_{2}^{2341} d_{2}+a_{3}^{2341} d_{3}+a_{4}^{2341} d_{1}+a_{12}^{2341} d_{1} d_{2}+a_{13}^{2341} d_{1} d_{3}+ \\
& a_{14}^{2341} d_{1} d_{4}+a_{23}^{2341} d_{2} d_{3}+a_{24}^{2341} d_{2} d_{4}+a_{34}^{2341} d_{3} d_{4}+a_{123}^{2341} d_{1} d_{2} d_{3}+a_{124}^{2341} d_{1} d_{2} d_{4}+ \\
& a_{134}^{2341} d_{1} d_{3} d_{4}+a_{234}^{2341} d_{2} d_{3} d_{4}+a_{1234}^{2341} d_{1} d_{2} d_{3} d_{4}, \\
& \theta^{2413}\left(d_{1}, d_{2}, d_{3}, d_{4}\right) \\
& =a^{2413}+a_{1}^{2413} d_{1}+a_{2}^{2413} d_{2}+a_{3}^{2413} d_{3}+a_{4}^{2413} d_{1}+a_{12}^{2413} d_{1} d_{2}+a_{13}^{2413} d_{1} d_{3}+ \\
& a_{14}^{2413} d_{1} d_{4}+a_{23}^{2413} d_{2} d_{3}+a_{24}^{2413} d_{2} d_{4}+a_{34}^{2413} d_{3} d_{4}+a_{123}^{2413} d_{1} d_{2} d_{3}+a_{124}^{2413} d_{1} d_{2} d_{4}+ \\
& a_{134}^{2413} d_{1} d_{3} d_{4}+a_{234}^{2413} d_{2} d_{3} d_{4}+a_{1234}^{2413} d_{1} d_{2} d_{3} d_{4} \text {, } \\
& \theta^{2431}\left(d_{1}, d_{2}, d_{3}, d_{4}\right) \\
& =a^{2431}+a_{1}^{2431} d_{1}+a_{2}^{2431} d_{2}+a_{3}^{2431} d_{3}+a_{4}^{2431} d_{1}+a_{12}^{2431} d_{1} d_{2}+a_{13}^{2431} d_{1} d_{3}+ \\
& a_{14}^{2431} d_{1} d_{4}+a_{23}^{2431} d_{2} d_{3}+a_{24}^{2431} d_{2} d_{4}+a_{34}^{2431} d_{3} d_{4}+a_{123}^{2431} d_{1} d_{2} d_{3}+a_{124}^{2431} d_{1} d_{2} d_{4}+ \\
& a_{134}^{2431} d_{1} d_{3} d_{4}+a_{234}^{2431} d_{2} d_{3} d_{4}+a_{1234}^{2431} d_{1} d_{2} d_{3} d_{4}, \\
& \theta^{3124}\left(d_{1}, d_{2}, d_{3}, d_{4}\right) \\
& =a^{3124}+a_{1}^{3124} d_{1}+a_{2}^{3124} d_{2}+a_{3}^{3124} d_{3}+a_{4}^{3124} d_{1}+a_{12}^{3124} d_{1} d_{2}+a_{13}^{3124} d_{1} d_{3}+ \\
& a_{14}^{3124} d_{1} d_{4}+a_{23}^{3124} d_{2} d_{3}+a_{24}^{3124} d_{2} d_{4}+a_{34}^{3124} d_{3} d_{4}+a_{123}^{3124} d_{1} d_{2} d_{3}+a_{124}^{3124} d_{1} d_{2} d_{4}+ \\
& a_{134}^{3124} d_{1} d_{3} d_{4}+a_{234}^{3124} d_{2} d_{3} d_{4}+a_{1234}^{3124} d_{1} d_{2} d_{3} d_{4}, \\
& \theta^{3142}\left(d_{1}, d_{2}, d_{3}, d_{4}\right) \\
& =a^{3142}+a_{1}^{3142} d_{1}+a_{2}^{3142} d_{2}+a_{3}^{3142} d_{3}+a_{4}^{3142} d_{1}+a_{12}^{3142} d_{1} d_{2}+a_{13}^{3142} d_{1} d_{3}+ \\
& a_{14}^{3142} d_{1} d_{4}+a_{23}^{3142} d_{2} d_{3}+a_{24}^{3142} d_{2} d_{4}+a_{34}^{3142} d_{3} d_{4}+a_{123}^{3142} d_{1} d_{2} d_{3}+a_{124}^{3142} d_{1} d_{2} d_{4}+ \\
& a_{134}^{3142} d_{1} d_{3} d_{4}+a_{234}^{3142} d_{2} d_{3} d_{4}+a_{1234}^{3142} d_{1} d_{2} d_{3} d_{4}, \\
& \theta^{3214}\left(d_{1}, d_{2}, d_{3}, d_{4}\right) \\
& =a^{3214}+a_{1}^{3214} d_{1}+a_{2}^{3214} d_{2}+a_{3}^{3214} d_{3}+a_{4}^{3214} d_{1}+a_{12}^{3214} d_{1} d_{2}+a_{13}^{3214} d_{1} d_{3}+ \\
& a_{14}^{3214} d_{1} d_{4}+a_{23}^{3214} d_{2} d_{3}+a_{24}^{3214} d_{2} d_{4}+a_{34}^{3214} d_{3} d_{4}+a_{123}^{3214} d_{1} d_{2} d_{3}+a_{124}^{3214} d_{1} d_{2} d_{4}+ \\
& a_{134}^{3214} d_{1} d_{3} d_{4}+a_{234}^{3214} d_{2} d_{3} d_{4}+a_{1234}^{3214} d_{1} d_{2} d_{3} d_{4}, \\
& \theta^{3241}\left(d_{1}, d_{2}, d_{3}, d_{4}\right) \\
& =a^{3241}+a_{1}^{3241} d_{1}+a_{2}^{3241} d_{2}+a_{3}^{3241} d_{3}+a_{4}^{3241} d_{1}+a_{12}^{3241} d_{1} d_{2}+a_{13}^{3241} d_{1} d_{3}+ \\
& a_{14}^{3241} d_{1} d_{4}+a_{23}^{3241} d_{2} d_{3}+a_{24}^{3241} d_{2} d_{4}+a_{34}^{3241} d_{3} d_{4}+a_{123}^{3241} d_{1} d_{2} d_{3}+a_{124}^{3241} d_{1} d_{2} d_{4}+ \\
& a_{134}^{3241} d_{1} d_{3} d_{4}+a_{234}^{3241} d_{2} d_{3} d_{4}+a_{1234}^{3241} d_{1} d_{2} d_{3} d_{4}, \\
& \theta^{3412}\left(d_{1}, d_{2}, d_{3}, d_{4}\right) \\
& =a^{3412}+a_{1}^{3412} d_{1}+a_{2}^{3412} d_{2}+a_{3}^{3412} d_{3}+a_{4}^{3412} d_{1}+a_{12}^{3412} d_{1} d_{2}+a_{13}^{3412} d_{1} d_{3}+ \\
& a_{14}^{3412} d_{1} d_{4}+a_{23}^{3412} d_{2} d_{3}+a_{24}^{3412} d_{2} d_{4}+a_{34}^{3412} d_{3} d_{4}+a_{123}^{3412} d_{1} d_{2} d_{3}+a_{124}^{3412} d_{1} d_{2} d_{4}+ \\
& a_{134}^{3412} d_{1} d_{3} d_{4}+a_{234}^{3412} d_{2} d_{3} d_{4}+a_{1234}^{3412} d_{1} d_{2} d_{3} d_{4},
\end{aligned}
$$




$$
\begin{aligned}
& \theta^{3421}\left(d_{1}, d_{2}, d_{3}, d_{4}\right) \\
& =a^{3421}+a_{1}^{3421} d_{1}+a_{2}^{3421} d_{2}+a_{3}^{3421} d_{3}+a_{4}^{3421} d_{1}+a_{12}^{3421} d_{1} d_{2}+a_{13}^{3421} d_{1} d_{3}+ \\
& a_{14}^{3421} d_{1} d_{4}+a_{23}^{3421} d_{2} d_{3}+a_{24}^{3421} d_{2} d_{4}+a_{34}^{3421} d_{3} d_{4}+a_{123}^{3421} d_{1} d_{2} d_{3}+a_{124}^{3421} d_{1} d_{2} d_{4}+ \\
& a_{134}^{3421} d_{1} d_{3} d_{4}+a_{234}^{3421} d_{2} d_{3} d_{4}+a_{1234}^{3421} d_{1} d_{2} d_{3} d_{4}, \\
& \theta^{4123}\left(d_{1}, d_{2}, d_{3}, d_{4}\right) \\
& =a^{4123}+a_{1}^{4123} d_{1}+a_{2}^{4123} d_{2}+a_{3}^{4123} d_{3}+a_{4}^{4123} d_{1}+a_{12}^{4123} d_{1} d_{2}+a_{13}^{4123} d_{1} d_{3}+ \\
& a_{14}^{4123} d_{1} d_{4}+a_{23}^{4123} d_{2} d_{3}+a_{24}^{4123} d_{2} d_{4}+a_{34}^{4123} d_{3} d_{4}+a_{123}^{4123} d_{1} d_{2} d_{3}+a_{124}^{4123} d_{1} d_{2} d_{4}+ \\
& a_{134}^{4123} d_{1} d_{3} d_{4}+a_{234}^{4123} d_{2} d_{3} d_{4}+a_{1234}^{4123} d_{1} d_{2} d_{3} d_{4} \text {, } \\
& \theta^{4132}\left(d_{1}, d_{2}, d_{3}, d_{4}\right) \\
& =a^{4132}+a_{1}^{4132} d_{1}+a_{2}^{4132} d_{2}+a_{3}^{4132} d_{3}+a_{4}^{4132} d_{1}+a_{12}^{4132} d_{1} d_{2}+a_{13}^{4132} d_{1} d_{3}+ \\
& a_{14}^{4132} d_{1} d_{4}+a_{23}^{4132} d_{2} d_{3}+a_{24}^{4132} d_{2} d_{4}+a_{34}^{4132} d_{3} d_{4}+a_{123}^{4132} d_{1} d_{2} d_{3}+a_{124}^{4132} d_{1} d_{2} d_{4}+ \\
& a_{134}^{4132} d_{1} d_{3} d_{4}+a_{234}^{4132} d_{2} d_{3} d_{4}+a_{1234}^{4132} d_{1} d_{2} d_{3} d_{4} \text {, } \\
& \theta^{4213}\left(d_{1}, d_{2}, d_{3}, d_{4}\right) \\
& =a^{4213}+a_{1}^{4213} d_{1}+a_{2}^{4213} d_{2}+a_{3}^{4213} d_{3}+a_{4}^{4213} d_{1}+a_{12}^{4213} d_{1} d_{2}+a_{13}^{4213} d_{1} d_{3}+ \\
& a_{14}^{4213} d_{1} d_{4}+a_{23}^{4213} d_{2} d_{3}+a_{24}^{4213} d_{2} d_{4}+a_{34}^{4213} d_{3} d_{4}+a_{123}^{4213} d_{1} d_{2} d_{3}+a_{124}^{4213} d_{1} d_{2} d_{4}+ \\
& a_{134}^{4213} d_{1} d_{3} d_{4}+a_{234}^{4213} d_{2} d_{3} d_{4}+a_{1234}^{4213} d_{1} d_{2} d_{3} d_{4}, \\
& \theta^{4231}\left(d_{1}, d_{2}, d_{3}, d_{4}\right) \\
& =a^{4231}+a_{1}^{4231} d_{1}+a_{2}^{4231} d_{2}+a_{3}^{4231} d_{3}+a_{4}^{4231} d_{1}+a_{12}^{4231} d_{1} d_{2}+a_{13}^{4231} d_{1} d_{3}+ \\
& a_{14}^{4231} d_{1} d_{4}+a_{23}^{4231} d_{2} d_{3}+a_{24}^{4231} d_{2} d_{4}+a_{34}^{4231} d_{3} d_{4}+a_{123}^{4231} d_{1} d_{2} d_{3}+a_{124}^{4231} d_{1} d_{2} d_{4}+ \\
& a_{134}^{4231} d_{1} d_{3} d_{4}+a_{234}^{4231} d_{2} d_{3} d_{4}+a_{1234}^{4231} d_{1} d_{2} d_{3} d_{4}, \\
& \theta^{4312}\left(d_{1}, d_{2}, d_{3}, d_{4}\right) \\
& =a^{4312}+a_{1}^{4312} d_{1}+a_{2}^{4312} d_{2}+a_{3}^{4312} d_{3}+a_{4}^{4312} d_{1}+a_{12}^{4312} d_{1} d_{2}+a_{13}^{4312} d_{1} d_{3}+ \\
& a_{14}^{4312} d_{1} d_{4}+a_{23}^{4312} d_{2} d_{3}+a_{24}^{4312} d_{2} d_{4}+a_{34}^{4312} d_{3} d_{4}+a_{123}^{4312} d_{1} d_{2} d_{3}+a_{124}^{4312} d_{1} d_{2} d_{4}+ \\
& a_{134}^{4312} d_{1} d_{3} d_{4}+a_{234}^{4312} d_{2} d_{3} d_{4}+a_{1234}^{4312} d_{1} d_{2} d_{3} d_{4} \text {, } \\
& \theta^{4321}\left(d_{1}, d_{2}, d_{3}, d_{4}\right) \\
& =a^{4321}+a_{1}^{4321} d_{1}+a_{2}^{4321} d_{2}+a_{3}^{4321} d_{3}+a_{4}^{4321} d_{1}+a_{12}^{4321} d_{1} d_{2}+a_{13}^{4321} d_{1} d_{3}+ \\
& a_{14}^{4321} d_{1} d_{4}+a_{23}^{4321} d_{2} d_{3}+a_{24}^{4321} d_{2} d_{4}+a_{34}^{4321} d_{3} d_{4}+a_{123}^{4321} d_{1} d_{2} d_{3}+a_{124}^{4321} d_{1} d_{2} d_{4}+ \\
& a_{134}^{4321} d_{1} d_{3} d_{4}+a_{234}^{4321} d_{2} d_{3} d_{4}+a_{1234}^{4321} d_{1} d_{2} d_{3} d_{4} \text {. }
\end{aligned}
$$

The conditions

$$
\begin{aligned}
& \theta^{1234} \circ g_{12}^{1234,1243}=\theta^{1243} \circ h_{12}^{1234,1243}, \theta^{1342} \circ g_{12}^{1342,1432}=\theta^{1432} \circ h_{12}^{1342,1432}, \\
& \theta^{2341} \circ g_{12}^{2341,2431}=\theta^{2431} \circ h_{12}^{2341,2431}, \theta^{3421} \circ g_{12}^{3421,4321}=\theta^{4321} \circ h_{12}^{3421,4321},
\end{aligned}
$$




$$
\begin{aligned}
& \theta^{2134} \circ g_{12}^{2134,2143}=\theta^{2143} \circ h_{12}^{2134,2143}, \theta^{3412} \circ g_{12}^{3412,4312}=\theta^{4312} \circ h_{12}^{3412,4312}, \\
& \theta^{1324} \circ g_{13}^{1324,1342}=\theta^{1342} \circ h_{13}^{1324,1342}, \theta^{1243} \circ g_{13}^{1243,1423}=\theta^{1423} \circ h_{13}^{1243,1423}, \\
& \theta^{3241} \circ g_{13}^{3241,3421}=\theta^{3421} \circ h_{13}^{3241,3421}, \theta^{2431} \circ g_{13}^{2431,4231}=\theta^{4231} \circ h_{13}^{2431,4231}, \\
& \theta^{3124} \circ g_{13}^{3124,3142}=\theta^{3142} \circ h_{13}^{3124,3142}, \theta^{2413} \circ g_{13}^{2413,4213}=\theta^{4213} \circ h_{13}^{2413,4213}, \\
& \theta^{1423} \circ g_{14}^{1423,1432}=\theta^{1432} \circ h_{14}^{1423,1432}, \theta^{1234} \circ g_{14}^{1234,1324}=\theta^{1324} \circ h_{14}^{1234,1324}, \\
& \theta^{4231} \circ g_{14}^{4231,4321}=\theta^{4321} \circ h_{14}^{4231,4321}, \theta^{2341} \circ g_{14}^{2341,3241}=\theta^{3241} \circ h_{14}^{2341,3241}, \\
& \theta^{4123} \circ g_{14}^{4123,4132}=\theta^{4132} \circ h_{14}^{4123,4132}, \theta^{2314} \circ g_{14}^{2314,3214}=\theta^{3214} \circ h_{14}^{2314,3214}, \\
& \theta^{2314} \circ g_{23}^{2314,2341}=\theta^{2341} \circ h_{23}^{2314,2341}, \theta^{2143} \circ g_{23}^{2143,2413}=\theta^{2413} \circ h_{23}^{2143,2413}, \\
& \theta^{3142} \circ g_{23}^{3142,3412}=\theta^{3412} \circ h_{23}^{3142,3412}, \theta^{1432} \circ g_{23}^{1432,4132}=\theta^{4132} \circ h_{23}^{1432,4132}, \\
& \theta^{3214} \circ g_{23}^{3214,3241}=\theta^{3241} \circ h_{23}^{3214,3241}, \theta^{1423} \circ g_{23}^{1423,4123}=\theta^{4123} \circ h_{23}^{1423,4123}, \\
& \theta^{2413} \circ g_{24}^{2413,2431}=\theta^{2431} \circ h_{24}^{2413,2431}, \theta^{2134} \circ g_{24}^{2134,2314}=\theta^{2314} \circ h_{24}^{2134,2314}, \\
& \theta^{4132} \circ g_{24}^{4132,4312}=\theta^{4312} \circ h_{24}^{4132,4312}, \theta^{1342} \circ g_{24}^{1342,3142}=\theta^{3142} \circ h_{24}^{1342,3142}, \\
& \theta^{4213} \circ g_{24}^{4213,4231}=\theta^{4231} \circ h_{24}^{4213,4231}, \theta^{1324} \circ g_{24}^{1324,3124}=\theta^{3124} \circ h_{24}^{1324,3124}, \\
& \theta^{3412} \circ g_{34}^{3412,3421}=\theta^{3421} \circ h_{34}^{3412,3421}, \theta^{3124} \circ g_{34}^{3124,3214}=\theta^{3214} \circ h_{34}^{3124,3214}, \\
& \theta^{4123} \circ g_{34}^{4123,4213}=\theta^{4213} \circ h_{34}^{4123,4213}, \theta^{1243} \circ g_{34}^{1243,2143}=\theta^{2143} \circ h_{34}^{1243,2143}, \\
& \theta^{4312} \circ g_{34}^{4312,4321}=\theta^{4321} \circ h_{34}^{4312,4321}, \theta^{1234} \circ g_{34}^{1234,2134}=\theta^{2134} \circ h_{34}^{1234,2134}
\end{aligned}
$$

are equivalent to the following conditions in terms of coefficients of the polynomials

$$
\begin{aligned}
& a^{1342}=a^{1432}, a_{1}^{1342}=a_{1}^{1432}, a_{2}^{1342}=a_{2}^{1432}, a_{3}^{1342}=a_{3}^{1432}, a_{4}^{1342}=a_{4}^{1432}, \\
& a_{12}^{1342}=a_{12}^{1432}, a_{13}^{1342}=a_{13}^{1432}, a_{14}^{1342}=a_{14}^{1432}, a_{23}^{1342}=a_{23}^{1432}, a_{24}^{1342}=a_{24}^{1432}, \\
& a_{123}^{1342}=a_{123}^{1432}, a_{124}^{1342}=a_{124}^{1432}, \\
& a^{2341}=a^{2431}, a_{1}^{2341}=a_{1}^{2431}, a_{2}^{2341}=a_{2}^{2431}, a_{3}^{2341}=a_{3}^{2431}, a_{4}^{2341}=a_{4}^{2431}, \\
& a_{12}^{2341}=a_{12}^{2431}, a_{13}^{2341}=a_{13}^{2431}, a_{14}^{2341}=a_{14}^{2431}, a_{23}^{2341}=a_{23}^{2431}, a_{24}^{2341}=a_{24}^{2431}, \\
& a_{123}^{2341}=a_{123}^{2431}, a_{124}^{2341}=a_{124}^{2431}, \\
& a^{3421}=a^{4321}, a_{1}^{3421}=a_{1}^{4321}, a_{2}^{3421}=a_{2}^{4321}, a_{3}^{3421}=a_{3}^{4321}, a_{4}^{3421}=a_{4}^{4321}, \\
& a_{12}^{3421}=a_{12}^{4321}, a_{13}^{3421}=a_{13}^{4321}, a_{14}^{3421}=a_{14}^{4321}, a_{23}^{3421}=a_{23}^{4321}, a_{24}^{3421}=a_{24}^{4321}, \\
& a_{123}^{3421}=a_{123}^{4321}, a_{124}^{3421}=a_{124}^{4321}, \\
& a^{2341}=a^{2314}, a_{1}^{2341}=a_{1}^{2314}, a_{2}^{2341}=a_{2}^{2314}, a_{3}^{2341}=a_{3}^{2314}, a_{4}^{2341}=a_{4}^{2314}, \\
& a_{12}^{2341}=a_{12}^{2314}, a_{13}^{2341}=a_{13}^{2314}, a_{14}^{2341}=a_{14}^{2314}, a_{24}^{2341}=a_{24}^{2314}, a_{34}^{2341}=a_{34}^{2314}, \\
& a_{124}^{2341}=a_{12}^{2314}, a_{134}^{2341}=a_{134}^{2314}, \\
& a^{2413}=a^{2143}, a_{1}^{2413}=a_{1}^{2143}, a_{2}^{2413}=a_{2}^{2143}, a_{3}^{2413}=a_{3}^{2143}, a_{4}^{2413}=a_{4}^{2143}, \\
& a_{12}^{2413}=a_{12}^{2143}, a_{13}^{2413}=a_{13}^{2143}, a_{14}^{2413}=a_{14}^{2143}, a_{24}^{2413}=a_{24}^{2143}, a_{34}^{2413}=a_{34}^{2143} \\
& a_{124}^{2413}=a_{124}^{2143}, a_{134}^{2413}=a_{134}^{2143},
\end{aligned}
$$




$$
\begin{aligned}
& a^{3412}=a^{3142}, a_{1}^{3412}=a_{1}^{3142}, a_{2}^{3412}=a_{2}^{3142}, a_{3}^{3412}=a_{3}^{3142}, a_{4}^{3412}=a_{4}^{3142}, \\
& a_{12}^{3412}=a_{12}^{3142}, a_{13}^{3412}=a_{13}^{3142}, a_{14}^{3412}=a_{14}^{3142}, a_{24}^{3412}=a_{24}^{3142}, a_{34}^{3412}=a_{34}^{3142}, \\
& a_{124}^{3412}=a_{124}^{3142}, a_{134}^{3412}=a_{134}^{3142}, \\
& a^{4132}=a^{1432}, a_{1}^{4132}=a_{1}^{1432}, a_{2}^{4132}=a_{2}^{1432}, a_{3}^{4132}=a_{3}^{1432}, a_{4}^{4132}=a_{4}^{1432}, \\
& a_{12}^{4132}=a_{12}^{1432}, a_{13}^{4132}=a_{13}^{1432}, a_{14}^{4132}=a_{14}^{1432}, a_{24}^{4132}=a_{24}^{1432}, a_{34}^{4132}=a_{34}^{1432}, \\
& a_{124}^{4132}=a_{124}^{1432}, a_{134}^{4132}=a_{134}^{1432}, \\
& a^{3412}=a^{3421}, a_{1}^{3412}=a_{1}^{3421}, a_{2}^{3412}=a_{2}^{3421}, a_{3}^{3412}=a_{3}^{3421}, a_{4}^{3412}=a_{4}^{3421}, \\
& a_{12}^{3412}=a_{12}^{3421}, a_{13}^{3412}=a_{13}^{3421}, a_{14}^{3412}=a_{14}^{3421}, a_{23}^{3412}=a_{23}^{3421}, a_{24}^{3412}=a_{24}^{3421}, \\
& a_{123}^{3412}=a_{123}^{3421}, a_{124}^{3412}=a_{124}^{3421}, \\
& a^{3124}=a^{3214}, a_{1}^{3124}=a_{1}^{3214}, a_{2}^{3124}=a_{2}^{3214}, a_{3}^{3124}=a_{3}^{3214}, a_{4}^{3124}=a_{4}^{3214}, \\
& a_{12}^{3124}=a_{12}^{3214}, a_{13}^{3124}=a_{13}^{3214}, a_{14}^{3124}=a_{14}^{3214}, a_{23}^{3124}=a_{23}^{3214}, a_{24}^{3124}=a_{24}^{3214}, \\
& a_{123}^{3124}=a_{123}^{3214}, a_{124}^{3124}=a_{124}^{3214}, \\
& a^{4123}=a^{4213}, a_{1}^{4123}=a_{1}^{4213}, a_{2}^{4123}=a_{2}^{4213}, a_{3}^{4123}=a_{3}^{4213}, a_{4}^{4123}=a_{4}^{4213}, \\
& a_{12}^{4123}=a_{12}^{4213}, a_{13}^{4123}=a_{13}^{4213}, a_{14}^{4123}=a_{14}^{4213}, a_{23}^{4123}=a_{23}^{4213}, a_{24}^{4123}=a_{24}^{4213} \text {, } \\
& a_{123}^{4123}=a_{123}^{4213}, a_{124}^{4123}=a_{124}^{4213}, \\
& a^{1243}=a^{2143}, a_{1}^{1243}=a_{1}^{2143}, a_{2}^{1243}=a_{2}^{2143}, a_{3}^{1243}=a_{3}^{2143}, a_{4}^{1243}=a_{4}^{2143}, \\
& a_{12}^{1243}=a_{12}^{2143}, a_{13}^{1243}=a_{13}^{2143}, a_{14}^{1243}=a_{14}^{2143}, a_{23}^{1243}=a_{23}^{2143}, a_{24}^{1243}=a_{24}^{2143}, \\
& a_{123}^{1243}=a_{123}^{2143}, a_{124}^{1243}=a_{124}^{2143}, \\
& a^{4123}=a^{4132}, a_{1}^{4123}=a_{1}^{4132}, a_{2}^{4123}=a_{2}^{4132}, a_{3}^{4123}=a_{3}^{4132}, a_{4}^{4123}=a_{4}^{4132}, \\
& a_{12}^{4123}=a_{12}^{4132}, a_{13}^{4123}=a_{13}^{4132}, a_{23}^{4123}=a_{23}^{4132}, a_{24}^{4123}=a_{24}^{4132}, a_{34}^{4123}=a_{34}^{4132}, \\
& a_{123}^{4123}=a_{123}^{4132}, a_{234}^{4123}=a_{234}^{4132}, \\
& a^{4231}=a^{4321}, a_{1}^{4231}=a_{1}^{4321}, a_{2}^{4231}=a_{2}^{4321}, a_{3}^{4231}=a_{3}^{4321}, a_{4}^{4231}=a_{4}^{4321}, \\
& a_{12}^{4231}=a_{12}^{4321}, a_{13}^{4231}=a_{13}^{4321}, a_{23}^{4231}=a_{23}^{4321}, a_{24}^{4231}=a_{24}^{4321}, a_{34}^{4231}=a_{34}^{4321}, \\
& a_{123}^{4231}=a_{123}^{4321}, a_{234}^{4231}=a_{234}^{4321}, \\
& a^{1234}=a^{1324}, a_{1}^{1234}=a_{1}^{1324}, a_{2}^{1234}=a_{2}^{1324}, a_{3}^{1234}=a_{3}^{1324}, a_{4}^{1234}=a_{4}^{1324}, \\
& a_{12}^{1234}=a_{12}^{1324}, a_{13}^{1234}=a_{13}^{1324}, a_{23}^{1234}=a_{23}^{1324}, a_{24}^{1234}=a_{24}^{1324}, a_{34}^{1234}=a_{34}^{1324} \text {, } \\
& a_{123}^{1234}=a_{123}^{1324}, a_{234}^{1234}=a_{234}^{1324} \text {, } \\
& a^{2314}=a^{3214}, a_{1}^{2314}=a_{1}^{3214}, a_{2}^{2314}=a_{2}^{3214}, a_{3}^{2314}=a_{3}^{3214}, a_{4}^{2314}=a_{4}^{3214}, \\
& a_{12}^{2314}=a_{12}^{3214}, a_{13}^{2314}=a_{13}^{3214}, a_{23}^{2314}=a_{23}^{3214}, a_{24}^{2314}=a_{24}^{3214}, a_{34}^{2314}=a_{34}^{3214}, \\
& a_{123}^{2314}=a_{123}^{3214}, a_{234}^{2314}=a_{234}^{3214}
\end{aligned}
$$


which can succinctly be summarized as

$$
\begin{aligned}
& a^{1234}=a^{1243}=a^{1324}=a^{1342}=a^{1423}=a^{1432}= \\
& a^{2134}=a^{2143}=a^{2314}=a^{2341}=a^{2413}=a^{2431}= \\
& a^{3124}=a^{3142}=a^{3214}=a^{3241}=a^{3412}=a^{3421}= \\
& a^{4123}=a^{4132}=a^{4213}=a^{4231}=a^{4312}=a^{4321} \text {, } \\
& a_{1}^{1234}=a_{1}^{1243}=a_{1}^{1324}=a_{1}^{1342}=a_{1}^{1423}=a_{1}^{1432}= \\
& a_{1}^{2134}=a_{1}^{2143}=a_{1}^{2314}=a_{1}^{2341}=a_{1}^{2413}=a_{1}^{2431}= \\
& a_{1}^{3124}=a_{1}^{3142}=a_{1}^{3214}=a_{1}^{3241}=a_{1}^{3412}=a_{1}^{3421}= \\
& a_{1}^{4123}=a_{1}^{4132}=a_{1}^{4213}=a_{1}^{4231}=a_{1}^{4312}=a_{1}^{4321} \text {, } \\
& a_{2}^{1234}=a_{2}^{1243}=a_{2}^{1324}=a_{2}^{1342}=a_{2}^{1423}=a_{2}^{1432}= \\
& a_{2}^{2134}=a_{2}^{2143}=a_{2}^{2314}=a_{2}^{2341}=a_{2}^{2413}=a_{2}^{2431}= \\
& a_{2}^{3124}=a_{2}^{3142}=a_{2}^{3214}=a_{2}^{3241}=a_{2}^{3412}=a_{2}^{3421}= \\
& a_{2}^{4123}=a_{2}^{4132}=a_{2}^{4213}=a_{2}^{4231}=a_{2}^{4312}=a_{2}^{4321} \text {, } \\
& a_{3}^{1234}=a_{3}^{1243}=a_{3}^{1324}=a_{3}^{1342}=a_{3}^{1423}=a_{3}^{1432}= \\
& a_{3}^{2134}=a_{3}^{2143}=a_{3}^{2314}=a_{3}^{2341}=a_{3}^{2413}=a_{3}^{2431}= \\
& a_{3}^{3124}=a_{3}^{3142}=a_{3}^{3214}=a_{3}^{3241}=a_{3}^{3412}=a_{3}^{3421}= \\
& a_{3}^{4123}=a_{3}^{4132}=a_{3}^{4213}=a_{3}^{4231}=a_{3}^{4312}=a_{3}^{4321} \text {, } \\
& a_{4}^{1234}=a_{4}^{1243}=a_{4}^{1324}=a_{4}^{1342}=a_{4}^{1423}=a_{4}^{1432}= \\
& a_{4}^{2134}=a_{4}^{2143}=a_{4}^{2314}=a_{4}^{2341}=a_{4}^{2413}=a_{4}^{2431}= \\
& a_{4}^{3124}=a_{4}^{3142}=a_{4}^{3214}=a_{4}^{3241}=a_{4}^{3412}=a_{4}^{3421}= \\
& a_{4}^{4123}=a_{4}^{4132}=a_{4}^{4213}=a_{4}^{4231}=a_{4}^{4312}=a_{4}^{4321} \text {, } \\
& a_{12}^{1234}=a_{12}^{1243}=a_{12}^{1324}=a_{12}^{1342}=a_{12}^{1423}=a_{12}^{1432}= \\
& a_{12}^{3124}=a_{12}^{3142}=a_{12}^{3412}=a_{12}^{4123}=a_{12}^{4132}=a_{12}^{4312} \text {, } \\
& a_{12}^{2134}=a_{12}^{2143}=a_{12}^{2314}=a_{12}^{2341}=a_{12}^{2413}=a_{12}^{2431}= \\
& a_{12}^{3214}=a_{12}^{3241}=a_{12}^{3421}=a_{12}^{4213}=a_{12}^{4231}=a_{12}^{4321} \text {, } \\
& a_{13}^{1342}=a_{13}^{1324}=a_{13}^{1432}=a_{13}^{1423}=a_{13}^{1234}=a_{13}^{1243}= \\
& a_{13}^{4132}=a_{13}^{4123}=a_{13}^{4213}=a_{13}^{2134}=a_{13}^{2143}=a_{13}^{2413} \text {, } \\
& a_{13}^{3142}=a_{13}^{3124}=a_{13}^{3412}=a_{13}^{3421}=a_{13}^{3214}=a_{13}^{3241}= \\
& a_{13}^{4312}=a_{13}^{4321}=a_{13}^{4231}=a_{13}^{2314}=a_{13}^{2341}=a_{13}^{2431} \text {, } \\
& a_{14}^{1423}=a_{14}^{1432}=a_{14}^{1243}=a_{14}^{1234}=a_{14}^{1342}=a_{14}^{1324}= \\
& a_{14}^{2143}=a_{14}^{2134}=a_{14}^{2314}=a_{14}^{3142}=a_{14}^{3124}=a_{14}^{3214} \text {, }
\end{aligned}
$$




$$
\begin{aligned}
& a_{14}^{4123}=a_{14}^{4132}=a_{14}^{4213}=a_{14}^{4231}=a_{14}^{4312}=a_{14}^{4321}= \\
& a_{14}^{2413}=a_{14}^{2431}=a_{14}^{2341}=a_{14}^{3412}=a_{14}^{3421}=a_{14}^{3241} \text {, } \\
& a_{23}^{2314}=a_{23}^{2341}=a_{23}^{2134}=a_{23}^{2143}=a_{23}^{2431}=a_{23}^{2413}= \\
& a_{23}^{1234}=a_{23}^{1243}=a_{23}^{1423}=a_{23}^{4231}=a_{23}^{4213}=a_{23}^{4123}, \\
& a_{23}^{3214}=a_{23}^{3241}=a_{23}^{3124}=a_{23}^{3142}=a_{23}^{3421}=a_{23}^{3412}= \\
& a_{23}^{1324}=a_{23}^{1342}=a_{23}^{1432}=a_{23}^{4321}=a_{23}^{4312}=a_{23}^{4132}, \\
& a_{24}^{2413}=a_{24}^{2431}=a_{24}^{2143}=a_{24}^{2134}=a_{24}^{2341}=a_{24}^{2314}= \\
& a_{24}^{1243}=a_{24}^{1234}=a_{24}^{1324}=a_{24}^{3241}=a_{24}^{3214}=a_{24}^{3124}, \\
& a_{24}^{4213}=a_{24}^{4231}=a_{24}^{4123}=a_{24}^{4132}=a_{24}^{4321}=a_{24}^{4312}= \\
& a_{24}^{1423}=a_{24}^{1432}=a_{24}^{1342}=a_{24}^{3421}=a_{24}^{3412}=a_{24}^{3142}, \\
& a_{34}^{3412}=a_{34}^{3421}=a_{34}^{3142}=a_{34}^{3124}=a_{34}^{3241}=a_{34}^{3214}= \\
& a_{34}^{1342}=a_{34}^{1324}=a_{34}^{1234}=a_{34}^{2341}=a_{34}^{2314}=a_{34}^{2134} \text {, } \\
& a_{34}^{4312}=a_{34}^{4321}=a_{34}^{4132}=a_{34}^{4123}=a_{34}^{4231}=a_{34}^{4213}= \\
& a_{34}^{1432}=a_{34}^{1423}=a_{34}^{1243}=a_{34}^{2431}=a_{34}^{2413}=a_{34}^{2143} \text {, } \\
& a_{123}^{1234}=a_{123}^{1243}=a_{123}^{1423}=a_{123}^{4123}, \\
& a_{123}^{1324}=a_{123}^{1342}=a_{123}^{1432}=a_{123}^{4132}, \\
& a_{123}^{2134}=a_{123}^{2143}=a_{123}^{2413}=a_{123}^{4213}, \\
& a_{123}^{2314}=a_{123}^{2341}=a_{123}^{2431}=a_{123}^{4231}, \\
& a_{123}^{3124}=a_{123}^{3142}=a_{123}^{3412}=a_{123}^{4312}, \\
& a_{123}^{3214}=a_{123}^{3241}=a_{123}^{3421}=a_{123}^{4321}, \\
& a_{124}^{1243}=a_{124}^{1234}=a_{124}^{1324}=a_{124}^{3124}, \\
& a_{124}^{1423}=a_{124}^{1432}=a_{124}^{1342}=a_{124}^{3142}, \\
& a_{124}^{2143}=a_{124}^{2134}=a_{124}^{2314}=a_{124}^{3214}, \\
& a_{124}^{2413}=a_{124}^{2431}=a_{124}^{2341}=a_{124}^{3241}, \\
& a_{124}^{4123}=a_{124}^{4132}=a_{124}^{4312}=a_{124}^{3412}, \\
& a_{124}^{4213}=a_{124}^{4231}=a_{124}^{4321}=a_{124}^{3421}, \\
& a_{134}^{1342}=a_{134}^{1324}=a_{134}^{1234}=a_{134}^{2134}, \\
& a_{134}^{1432}=a_{134}^{1423}=a_{134}^{1243}=a_{134}^{2143}, \\
& a_{134}^{3142}=a_{134}^{3124}=a_{134}^{3214}=a_{134}^{2314}, \\
& a_{134}^{3412}=a_{134}^{3421}=a_{134}^{3241}=a_{134}^{2341}, \\
& a_{134}^{4132}=a_{134}^{4123}=a_{134}^{4213}=a_{134}^{2413} \text {, }
\end{aligned}
$$




$$
\begin{aligned}
& a_{134}^{4312}=a_{134}^{4321}=a_{134}^{4231}=a_{134}^{2431}, \\
& a_{234}^{2341}=a_{234}^{2314}=a_{234}^{2134}=a_{234}^{1234}, \\
& a_{234}^{2341}=a_{234}^{2413}=a_{234}^{2143}=a_{234}^{1243}, \\
& a_{234}^{3241}=a_{234}^{3214}=a_{234}^{3124}=a_{234}^{1324}, \\
& a_{234}^{3421}=a_{234}^{3412}=a_{234}^{3142}=a_{234}^{1342}, \\
& a_{234}^{4231}=a_{234}^{4213}=a_{234}^{4123}=a_{234}^{1423}, \\
& a_{234}^{4321}=a_{234}^{4312}=a_{234}^{4132}=a_{234}^{1432} .
\end{aligned}
$$

Therefore, there exists a unique mapping $\theta: P \rightarrow \mathbb{R}$ such that

$$
\begin{aligned}
& \theta^{1234}=\theta \circ f_{1234}, \theta^{1243}=\theta \circ f_{1243}, \theta^{1324}=\theta \circ f_{1324}, \\
& \theta^{1342}=\theta \circ f_{1342}, \theta^{1423}=\theta \circ f_{1423}, \theta^{1432}=\theta \circ f_{1432}, \\
& \theta^{2134}=\theta \circ f_{2134}, \theta^{2143}=\theta \circ f_{2143}, \theta^{2314}=\theta \circ f_{2314}, \\
& \theta^{2341}=\theta \circ f_{2341}, \theta^{2413}=\theta \circ f_{2413}, \theta^{2431}=\theta \circ f_{2431}, \\
& \theta^{3124}=\theta \circ f_{3124}, \theta^{3142}=\theta \circ f_{3142}, \theta^{3214}=\theta \circ f_{3214}, \\
& \theta^{3241}=\theta \circ f_{3241}, \theta^{3412}=\theta \circ f_{3412}, \theta^{3421}=\theta \circ f_{3421}, \\
& \theta^{4123}=\theta \circ f_{4123}, \theta^{4132}=\theta \circ f_{4132}, \theta^{4213}=\theta \circ f_{4213}, \\
& \theta^{4231}=\theta \circ f_{4231}, \theta^{4312}=\theta \circ f_{4312}, \theta^{4321}=\theta \circ f_{4321} .
\end{aligned}
$$

The proof is now complete.

Remark 3.2. For our convenience, we display the positions $5-30$ of $f_{i j k l}\left(d_{1}, d_{2}, d_{3}, d_{4}\right)$ as tables as follows:

\begin{tabular}{ccccccc}
\hline & $5 / d_{1} d_{2}$ & $6 / d_{1} d_{3}$ & $7 / d_{1} d_{4}$ & $8 / d_{2} d_{3}$ & $9 / d_{2} d_{4}$ & $10 / d_{3} d_{4}$ \\
\hline 1234 & 0 & 0 & 0 & 0 & 0 & 0 \\
1243 & 0 & 0 & 0 & 0 & 0 & $d_{3} d_{4}$ \\
1324 & 0 & 0 & 0 & $d_{2} d_{3}$ & 0 & 0 \\
1342 & 0 & 0 & 0 & $d_{2} d_{3}$ & $d_{2} d_{4}$ & 0 \\
1423 & 0 & 0 & 0 & 0 & $d_{2} d_{4}$ & $d_{3} d_{4}$ \\
1432 & 0 & 0 & 0 & $d_{2} d_{3}$ & $d_{2} d_{4}$ & $d_{3} d_{4}$ \\
2134 & $d_{1} d_{2}$ & 0 & 0 & 0 & 0 & 0 \\
2143 & $d_{1} d_{2}$ & 0 & 0 & 0 & 0 & $d_{3} d_{4}$ \\
2314 & $d_{1} d_{2}$ & $d_{1} d_{3}$ & 0 & 0 & 0 & 0 \\
2341 & $d_{1} d_{2}$ & $d_{1} d_{3}$ & $d_{1} d_{4}$ & 0 & 0 & 0 \\
2413 & $d_{1} d_{2}$ & 0 & $d_{1} d_{4}$ & 0 & 0 & $d_{3} d_{4}$ \\
2431 & $d_{1} d_{2}$ & $d_{1} d_{3}$ & $d_{1} d_{4}$ & 0 & 0 & $d_{3} d_{4}$ \\
3124 & 0 & $d_{1} d_{3}$ & 0 & $d_{2} d_{3}$ & 0 & 0 \\
3142 & 0 & $d_{1} d_{3}$ & 0 & $d_{2} d_{3}$ & $d_{2} d_{4}$ & 0 \\
3214 & $d_{1} d_{2}$ & $d_{1} d_{3}$ & 0 & $d_{2} d_{3}$ & 0 & 0 \\
3241 & $d_{1} d_{2}$ & $d_{1} d_{3}$ & $d_{1} d_{4}$ & $d_{2} d_{3}$ & 0 & 0 \\
3412 & 0 & $d_{1} d_{3}$ & $d_{1} d_{4}$ & $d_{2} d_{3}$ & $d_{2} d_{4}$ & 0 \\
3421 & $d_{1} d_{2}$ & $d_{1} d_{3}$ & $d_{1} d_{4}$ & $d_{2} d_{3}$ & $d_{2} d_{4}$ & 0
\end{tabular}


$\begin{array}{lllllll}4123 & 0 & 0 & d_{1} d_{4} & 0 & d_{2} d_{4} & d_{3} d_{4}\end{array}$ $\begin{array}{lllllll}4132 & 0 & 0 & d_{1} d_{4} & d_{2} d_{3} & d_{2} d_{4} & d_{3} d_{4}\end{array}$ $\begin{array}{lllllll}4213 & d_{1} d_{2} & 0 & d_{1} d_{4} & 0 & d_{2} d_{4} & d_{3} d_{4}\end{array}$ $\begin{array}{lllllll}4231 & d_{1} d_{2} & d_{1} d_{3} & d_{1} d_{4} & 0 & d_{2} d_{4} & d_{3} d_{4}\end{array}$ $\begin{array}{lllllll}4312 & 0 & d_{1} d_{3} & d_{1} d_{4} & d_{2} d_{3} & d_{2} d_{4} & d_{3} d_{4}\end{array}$ $\begin{array}{lllllll}4321 & d_{1} d_{2} & d_{1} d_{3} & d_{1} d_{4} & d_{2} d_{3} & d_{2} d_{4} & d_{3} d_{4}\end{array}$

\begin{tabular}{|c|c|c|c|c|}
\hline & $11-15 / d_{1} d_{2} d_{3}$ & $16-20 / d_{1} d_{2} d_{4}$ & $21-25 / d_{1} d_{3} d_{4}$ & $26-30 / d_{2} d_{3} d_{4}$ \\
\hline \multicolumn{5}{|l|}{1234} \\
\hline 1243 & & & 21 & 26 \\
\hline 1324 & 11 & & & 27 \\
\hline 1342 & 11 & 16 & & 28 \\
\hline 1423 & & 16 & 21 & 29 \\
\hline 1432 & 11 & 16 & 21 & 30 \\
\hline 2134 & 12 & 17 & & \\
\hline 2143 & 12 & 17 & 21 & 26 \\
\hline 2314 & 13 & 17 & 22 & \\
\hline 2341 & 13 & 18 & 23 & \\
\hline 2413 & 12 & 18 & 24 & 26 \\
\hline 2431 & 13 & 18 & 25 & 26 \\
\hline 3124 & 14 & & 22 & 27 \\
\hline 3142 & 14 & 16 & 22 & 28 \\
\hline 3214 & 15 & 17 & 22 & 27 \\
\hline 3241 & 15 & 18 & 23 & 27 \\
\hline 3412 & 14 & 19 & 23 & 28 \\
\hline 3421 & 15 & 20 & 23 & 28 \\
\hline 4123 & & 19 & 24 & 29 \\
\hline 4132 & 11 & 19 & 24 & 30 \\
\hline 4213 & 12 & 20 & 24 & 29 \\
\hline 4231 & 13 & 20 & 25 & 29 \\
\hline 4312 & 14 & 19 & 25 & 30 \\
\hline 4321 & 15 & 20 & 25 & 30 \\
\hline
\end{tabular}

Corollary 3.3. Let $M$ be a microlinear space with mappings

$$
\begin{aligned}
& \gamma_{1234}: Q^{1234} \rightarrow M, \gamma_{1243}: Q^{1243} \rightarrow M, \gamma_{1324}: Q^{1324} \rightarrow M, \\
& \gamma_{1342}: Q^{1342} \rightarrow M, \gamma_{1423}: Q^{1423} \rightarrow M, \gamma_{1432}: Q^{1432} \rightarrow M, \\
& \gamma_{2134}: Q^{2134} \rightarrow M, \gamma_{2143}: Q^{2143} \rightarrow M, \gamma_{2314}: Q^{2314} \rightarrow M, \\
& \gamma_{2341}: Q^{2341} \rightarrow M, \gamma_{2413}: Q^{2413} \rightarrow M, \gamma_{2431}: Q^{2431} \rightarrow M, \\
& \gamma_{3124}: Q^{3124} \rightarrow M, \gamma_{3142}: Q^{3142} \rightarrow M, \gamma_{3214}: Q^{3214} \rightarrow M, \\
& \gamma_{3241}: Q^{3241} \rightarrow M, \gamma_{3412}: Q^{3412} \rightarrow M, \gamma_{3421}: Q^{3421} \rightarrow M, \\
& \gamma_{4123}: Q^{4123} \rightarrow M, \gamma_{4132}: Q^{4132} \rightarrow M, \gamma_{4213}: Q^{4213} \rightarrow M, \\
& \gamma_{4231}: Q^{4231} \rightarrow M, \gamma_{4312}: Q^{4312} \rightarrow M, \gamma_{4321}: Q^{4321} \rightarrow M
\end{aligned}
$$


abiding by

$$
\begin{aligned}
& \gamma_{1234} \circ g_{12}^{1234,1243}=\gamma_{1243} \circ h_{12}^{1234,1243}, \gamma_{1342} \circ g_{12}^{1342,1432}=\gamma_{1432} \circ h_{12}^{1342,1432}, \\
& \gamma_{2341} \circ g_{12}^{2341,2431}=\gamma_{2431} \circ h_{12}^{2341,2431}, \gamma_{3421} \circ g_{12}^{3421,4321}=\gamma_{4321} \circ h_{12}^{3421,4321}, \\
& \gamma_{2134} \circ g_{12}^{2134,2143}=\gamma_{2143} \circ h_{12}^{2134,2143}, \gamma_{3412} \circ g_{12}^{3412,4312}=\gamma_{4312} \circ h_{12}^{3412,4312}, \\
& \gamma_{1324} \circ g_{13}^{1324,1342}=\gamma_{1342} \circ h_{13}^{1324,1342}, \gamma_{1243} \circ g_{13}^{1243,1423}=\gamma_{1423} \circ h_{13}^{1243,1423}, \\
& \gamma_{3241} \circ g_{13}^{3241,3421}=\gamma_{3421} \circ h_{13}^{3241,3421}, \gamma_{2431} \circ g_{13}^{2431,4231}=\gamma_{4231} \circ h_{13}^{2431,4231}, \\
& \gamma_{3124} \circ g_{13}^{3124,3142}=\gamma_{3142} \circ h_{13}^{3124,3142}, \gamma_{2413} \circ g_{13}^{2413,4213}=\gamma_{4213} \circ h_{13}^{2413,4213}, \\
& \gamma_{1423} \circ g_{14}^{1423,1432}=\gamma_{1432} \circ h_{14}^{1423,1432}, \gamma_{1234} \circ g_{14}^{1234,1324}=\gamma_{1324} \circ h_{14}^{1234,1324}, \\
& \gamma_{4231} \circ g_{14}^{4231,4321}=\gamma_{4321} \circ h_{14}^{4231,4321}, \gamma_{2341} \circ g_{14}^{2341,3241}=\gamma_{3241} \circ h_{14}^{2341,3241}, \\
& \gamma_{4123} \circ g_{14}^{4123,4132}=\gamma_{4132} \circ h_{14}^{4123,4132}, \gamma_{2314} \circ g_{14}^{2314,3214}=\gamma_{3214} \circ h_{14}^{2314,3214}, \\
& \gamma_{2314} \circ g_{23}^{2314,2341}=\gamma_{2341} \circ h_{23}^{2314,2341}, \gamma_{2143} \circ g_{23}^{2143,2413}=\gamma_{2413} \circ h_{23}^{2143,2413}, \\
& \gamma_{3142} \circ g_{23}^{3142,3412}=\gamma_{3412} \circ h_{23}^{3142,3412}, \gamma_{1432} \circ g_{23}^{1432,4132}=\gamma_{4132} \circ h_{23}^{1432,4132}, \\
& \gamma_{3214} \circ g_{23}^{3214,3241}=\gamma_{3241} \circ h_{23}^{3214,3241}, \gamma_{1423} \circ g_{23}^{1423,4123}=\gamma_{4123} \circ h_{23}^{1423,4123}, \\
& \gamma_{2413} \circ g_{24}^{2413,2431}=\gamma_{2431} \circ h_{24}^{2413,2431}, \gamma_{2134} \circ g_{24}^{2134,2314}=\gamma_{2314} \circ h_{24}^{2134,2314}, \\
& \gamma_{4132} \circ g_{24}^{4132,4312}=\gamma_{4312} \circ h_{24}^{4132,4312}, \gamma_{1342} \circ g_{24}^{1342,3142}=\gamma_{3142} \circ h_{24}^{1342,3142}, \\
& \gamma_{4213} \circ g_{24}^{4213,4231}=\gamma_{4231} \circ h_{24}^{4213,4231}, \gamma_{1324} \circ g_{24}^{1324,3124}=\gamma_{3124} \circ h_{24}^{1324,3124}, \\
& \gamma_{3412} \circ g_{34}^{3412,3421}=\gamma_{3421} \circ h_{34}^{3412,3421}, \gamma_{3124} \circ g_{34}^{3124,3214}=\gamma_{3214} \circ h_{34}^{3124,3214}, \\
& \gamma_{4123} \circ g_{34}^{4123,4213}=\gamma_{4213} \circ h_{34}^{4123,4213}, \gamma_{1243} \circ g_{34}^{1243,2143}=\gamma_{2143} \circ h_{34}^{1243,2143}, \\
& \gamma_{4312} \circ g_{34}^{4312,4321}=\gamma_{4321} \circ h_{34}^{4312,4321}, \gamma_{1234} \circ g_{34}^{1234,2134}=\gamma_{2134} \circ h_{34}^{1234,2134}
\end{aligned}
$$

Then, there exists a unique mapping

$$
\mathfrak{m}: P \rightarrow M
$$

such that

$$
\begin{aligned}
& \mathfrak{m} \circ f_{1234}=\gamma_{1234}, \mathfrak{m} \circ f_{1243}=\gamma_{1243}, \mathfrak{m} \circ f_{1324}=\gamma_{1324}, \mathfrak{m} \circ f_{1342}=\gamma_{1342}, \\
& \mathfrak{m} \circ f_{1423}=\gamma_{1423}, \mathfrak{m} \circ f_{1432}=\gamma_{1432}, \mathfrak{m} \circ f_{2134}=\gamma_{2134}, \mathfrak{m} \circ f_{2143}=\gamma_{2143} \text {, } \\
& \mathfrak{m} \circ f_{2314}=\gamma_{2314}, \mathfrak{m} \circ f_{2341}=\gamma_{2341}, \mathfrak{m} \circ f_{2413}=\gamma_{2413}, \mathfrak{m} \circ f_{2431}=\gamma_{2431} \text {, } \\
& \mathfrak{m} \circ f_{3124}=\gamma_{3124}, \mathfrak{m} \circ f_{3142}=\gamma_{3142}, \mathfrak{m} \circ f_{3214}=\gamma_{3214}, \mathfrak{m} \circ f_{3241}=\gamma_{3241} \text {, } \\
& \mathfrak{m} \circ f_{3412}=\gamma_{3412}, \mathfrak{m} \circ f_{3421}=\gamma_{3421}, \mathfrak{m} \circ f_{4123}=\gamma_{4123}, \mathfrak{m} \circ f_{4132}=\gamma_{4132} \text {, } \\
& \mathfrak{m} \circ f_{4213}=\gamma_{4213}, \mathfrak{m} \circ f_{4231}=\gamma_{4231}, \mathfrak{m} \circ f_{4312}=\gamma_{4312}, \mathfrak{m} \circ f_{4321}=\gamma_{4321} \text {. }
\end{aligned}
$$

Theorem 3.4. Let $M$ be a microlinear space. Let

$\gamma_{1234}, \gamma_{1243}, \gamma_{1324}, \gamma_{1342}, \gamma_{1423}, \gamma_{1432}, \gamma_{2134}, \gamma_{2143}$

$\gamma_{2314}, \gamma_{2341}, \gamma_{2413}, \gamma_{2431}, \gamma_{3124}, \gamma_{3142}, \gamma_{3214}, \gamma_{3241},: D^{4} \rightarrow M$

$\gamma_{3124}, \gamma_{3142}, \gamma_{4123}, \gamma_{4132}, \gamma_{4213}, \gamma_{4231}, \gamma_{4312}, \gamma_{4321}$

with

$$
\gamma_{1234}\left|D^{4}\{(3,4)\}=\gamma_{1243}\right| D^{4}\{(3,4)\}, \gamma_{1342}\left|D^{4}\{(3,4)\}=\gamma_{1432}\right| D^{4}\{(3,4)\},
$$


$\gamma_{2341}\left|D^{4}\{(3,4)\}=\gamma_{2431}\right| D^{4}\{(3,4)\}, \gamma_{3421}\left|D^{4}\{(3,4)\}=\gamma_{4321}\right| D^{4}\{(3,4)\}$, $\gamma_{2143}\left|D^{4}\{(3,4)\}=\gamma_{2134}\right| D^{4}\{(3,4)\}, \gamma_{4312}\left|D^{4}\{(3,4)\}=\gamma_{3412}\right| D^{4}\{(3,4)\}$, $\gamma_{1342}\left|D^{4}\{(2,4)\}=\gamma_{1324}\right| D^{4}\{(2,4)\}, \gamma_{1423}\left|D^{4}\{(2,4)\}=\gamma_{1243}\right| D^{4}\{(2,4)\}$, $\gamma_{3421}\left|D^{4}\{(2,4)\}=\gamma_{3241}\right| D^{4}\{(2,4)\}, \gamma_{4231}\left|D^{4}\{(2,4)\}=\gamma_{2431}\right| D^{4}\{(2,4)\}$, $\gamma_{3124}\left|D^{4}\{(2,4)\}=\gamma_{3142}\right| D^{4}\{(2,4)\}, \gamma_{2413}\left|D^{4}\{(2,4)\}=\gamma_{4213}\right| D^{4}\{(2,4)\}$, $\gamma_{1423}\left|D^{4}\{(2,3)\}=\gamma_{1432}\right| D^{4}\{(2,3)\}, \gamma_{1234}\left|D^{4}\{(2,3)\}=\gamma_{1324}\right| D^{4}\{(2,3)\}$, $\gamma_{4231}\left|D^{4}\{(2,3)\}=\gamma_{4321}\right| D^{4}\{(2,3)\}, \gamma_{2341}\left|D^{4}\{(2,3)\}=\gamma_{3241}\right| D^{4}\{(2,3)\}$, $\gamma_{4132}\left|D^{4}\{(2,3)\}=\gamma_{4123}\right| D^{4}\{(2,3)\}, \gamma_{3214}\left|D^{4}\{(2,3)\}=\gamma_{2314}\right| D^{4}\{(2,3)\}$, $\gamma_{2314}\left|D^{4}\{(1,4)\}=\gamma_{2341}\right| D^{4}\{(1,4)\}, \gamma_{2143}\left|D^{4}\{(1,4)\}=\gamma_{2413}\right| D^{4}\{(1,4)\}$, $\gamma_{3142}\left|D^{4}\{(1,4)\}=\gamma_{3412}\right| D^{4}\{(1,4)\}, \gamma_{1432}\left|D^{4}\{(1,4)\}=\gamma_{4132}\right| D^{4}\{(1,4)\}$, $\gamma_{3241}\left|D^{4}\{(1,4)\}=\gamma_{3214}\right| D^{4}\{(1,4)\}, \gamma_{4123}\left|D^{4}\{(1,4)\}=\gamma_{1423}\right| D^{4}\{(1,4)\}$, $\gamma_{2431}\left|D^{4}\{(1,3)\}=\gamma_{2413}\right| D^{4}\{(1,3)\}, \gamma_{2314}\left|D^{4}\{(1,3)\}=\gamma_{2134}\right| D^{4}\{(1,3)\}$, $\gamma_{4312}\left|D^{4}\{(1,3)\}=\gamma_{4132}\right| D^{4}\{(1,3)\}, \gamma_{3142}\left|D^{4}\{(1,3)\}=\gamma_{1342}\right| D^{4}\{(1,3)\}$, $\gamma_{4213}\left|D^{4}\{(1,3)\}=\gamma_{4231}\right| D^{4}\{(1,3)\}, \gamma_{1324}\left|D^{4}\{(1,3)\}=\gamma_{3124}\right| D^{4}\{(1,3)\}$, $\gamma_{3412}\left|D^{4}\{(1,2)\}=\gamma_{3421}\right| D^{4}\{(1,2)\}, \gamma_{3124}\left|D^{4}\{(1,2)\}=\gamma_{3214}\right| D^{4}\{(1,2)\}$, $\gamma_{4123}\left|D^{4}\{(1,2)\}=\gamma_{4213}\right| D^{4}\{(1,2)\}, \gamma_{1243}\left|D^{4}\{(1,2)\}=\gamma_{2143}\right| D^{4}\{(1,2)\}$, $\gamma_{4321}\left|D^{4}\{(1,2)\}=\gamma_{4312}\right| D^{4}\{(1,2)\}, \gamma_{2134}\left|D^{4}\{(1,2)\}=\gamma_{1234}\right| D^{4}\{(1,2)\}$.

Then, we have

$$
\begin{aligned}
& {\left[\left[\left[\gamma_{1234} \frac{\cdot}{12} \gamma_{1243}\right]-\frac{-}{1}\left[\gamma_{1342} \frac{\dot{1}}{12} \gamma_{1432}\right]\right]-\left[\left[\gamma_{2341} \frac{\dot{1}}{12} \gamma_{2431}\right] \cdot \frac{\dot{1}}{1}\left[\gamma_{3421} \frac{\dot{-}}{12} \gamma_{4321}\right]\right]\right]+} \\
& {\left[\left[\left[\gamma_{1342} \frac{\bullet}{13} \gamma_{1324}\right]-\frac{\dot{1}}{1}\left[\gamma_{1423} \frac{\dot{-}}{13} \gamma_{1243}\right]\right]-\left[\left[\gamma_{3421} \frac{\dot{1}}{13} \gamma_{3241}\right] \dot{-}\left[\gamma_{4231} \frac{\dot{1}}{13} \gamma_{2431}\right]\right]\right]+}
\end{aligned}
$$

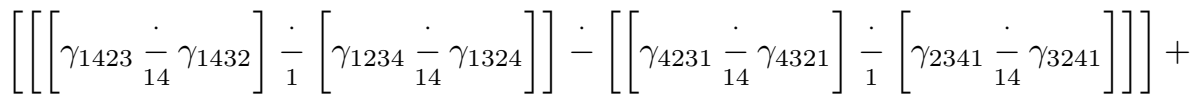

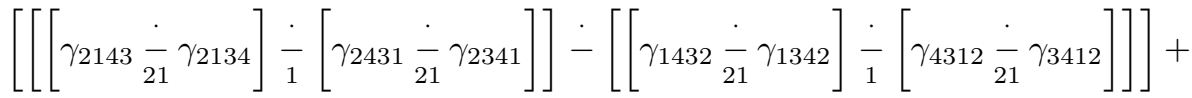

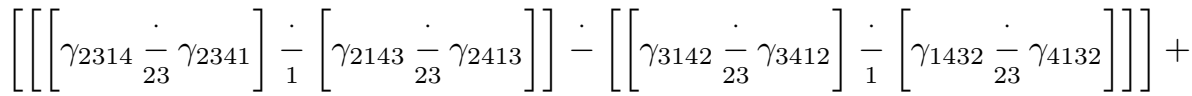

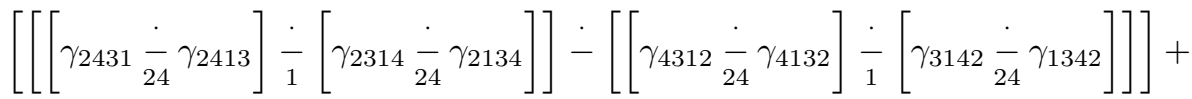

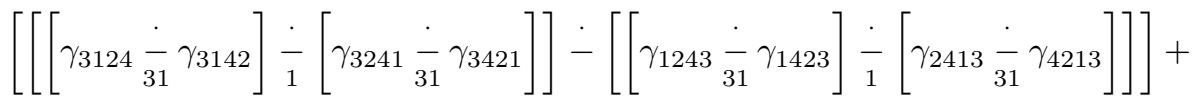

$$
\begin{aligned}
& {\left[\left[\left[\gamma_{3241} \frac{\cdot}{32} \gamma_{3214}\right]-\left[\gamma_{3412} \frac{\cdot}{32} \gamma_{3142}\right]\right]-\left[\left[\gamma_{2413} \frac{\cdot}{32} \gamma_{2143}\right] \cdot\left[\gamma_{4123} \frac{\cdot}{32} \gamma_{1423}\right]\right]\right]+} \\
& {\left[\left[\left[\gamma_{3412} \frac{\dot{3}}{34} \gamma_{3421}\right]-\left[\gamma_{3124} \frac{\dot{-}}{34} \gamma_{3214}\right]\right]-\left[\left[\gamma_{4123} \frac{\dot{3}}{34} \gamma_{4213}\right]-\left[\gamma_{1243} \frac{\dot{3}}{34} \gamma_{2143}\right]\right]\right]+}
\end{aligned}
$$




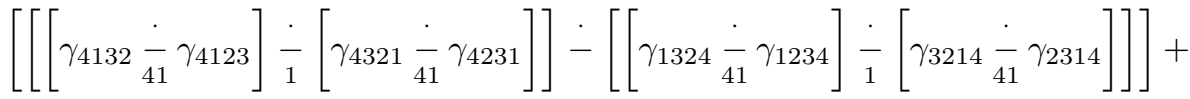

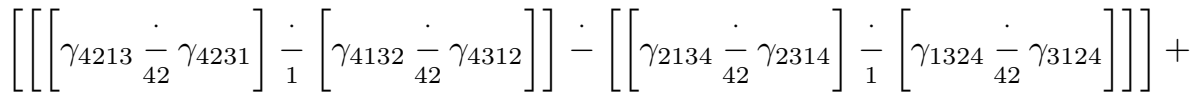

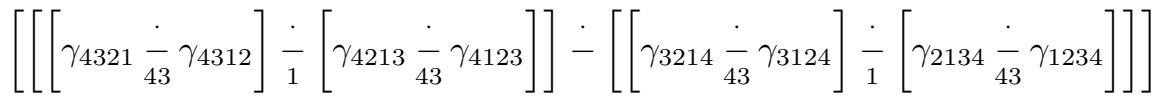

$$
\begin{aligned}
& =0 \text {. }
\end{aligned}
$$

Proof. The proof is divided into thirteen steps.

(1) Since

$$
\gamma_{1234}\left(d_{1}, d_{2}, d_{3}, d_{4}\right)=\mathfrak{m}\left(d_{1}, d_{2}, d_{3}, d_{4},{ }_{5}, \ldots, 0\right)
$$

and

$$
\begin{aligned}
& \gamma_{1243}\left(d_{1}, d_{2}, d_{3}, d_{4}\right) \\
& =\mathfrak{m}\left(\begin{array}{c}
d_{1}, d_{2}, d_{3}, d_{4}, \underset{5}{0}, \ldots, \underset{9}{0}, d_{3} d_{4}, \underset{11}{0}, \ldots, \underset{20}{0}, d_{1} d_{3} d_{4}, \underset{22}{0}, \ldots, \underset{25}{0}, d_{2} d_{3} d_{4}, \underset{27}{0}, \ldots, \underset{30}{0}, d_{1} d_{2} d_{3} d_{4}, \underset{32}{0}, \ldots, \underset{53}{0}
\end{array}\right),
\end{aligned}
$$

we have

$$
\begin{aligned}
& \left(\gamma_{1234}-\gamma_{1243}\right)\left(d_{1}, d_{2}, d_{3}\right) \\
& =\mathfrak{m}\left(\begin{array}{c}
d_{1}, d_{2}, \underset{3}{0}, \ldots, \underset{9}{0},-d_{3}, \underset{11}{0}, \ldots, \underset{20}{0},-d_{1} d_{3}, \underset{22}{0}, \ldots, \underset{25}{0},-d_{2} d_{3}, \underset{27}{0}, \ldots, \underset{30}{0}-d_{1} d_{2} d_{3}, \underset{32}{0}, \ldots, \underset{53}{0}
\end{array}\right) .
\end{aligned}
$$

For we have

$$
\begin{aligned}
& \mathfrak{n}_{\left(\gamma_{1234}, \gamma_{1243}\right)}^{4}\left(d_{1}, d_{2}, d_{3}, d_{4}, d_{5}\right) \\
& =\mathfrak{m}\left(\begin{array}{c}
d_{1}, d_{2}, d_{3}, d_{4}, 0, \ldots, \underset{5}{0}, d_{3} d_{4}-d_{5}, \underset{11}{0}, \ldots, \underset{20}{0}, d_{1} d_{3} d_{4}-d_{1} d_{5}, \underset{22}{0}, \ldots, \underset{25}{0}, \\
d_{2} d_{3} d_{4}-d_{2} d_{5}, \underset{27}{0}, \ldots,{ }_{30}^{0}, d_{1} d_{2} d_{3} d_{4}-d_{1} d_{2} d_{5}, \underset{32}{0}, \ldots, \underset{53}{0}
\end{array}\right) .
\end{aligned}
$$

Since

$$
\begin{aligned}
& \gamma_{1342}\left(d_{1}, d_{2}, d_{3}, d_{4}\right) \\
& =\mathfrak{m}\left(\begin{array}{c}
d_{1}, d_{2}, d_{3}, d_{4}, \underset{5}{0}, \ldots, \underset{7}{0}, d_{2} d_{3}, d_{2} d_{4}, \underset{10}{0}, d_{1} d_{2} d_{3}, \underset{12}{0}, \ldots, \underset{15}{0}, d_{1} d_{2} d_{4}, \underset{17}{0}, \ldots, \underset{27}{0}, \\
d_{2} d_{3} d_{4}, 0,0,0,0, d_{1} d_{2} d_{3} d_{4}, \underset{34}{0}, \ldots,{ }_{53}^{0}
\end{array}\right)
\end{aligned}
$$

$\gamma_{1432}\left(d_{1}, d_{2}, d_{3}, d_{4}\right)$

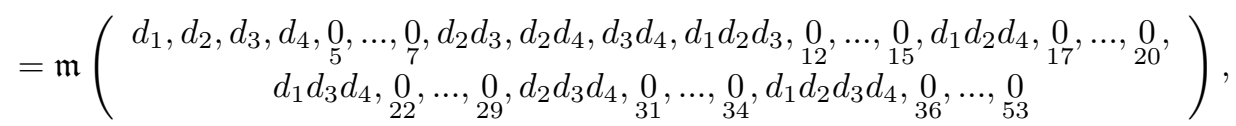

we have

$$
\left(\gamma_{1342} \frac{-}{12} \gamma_{1432}\right)\left(d_{1}, d_{2}, d_{3}\right)
$$




$$
=\mathfrak{m}\left(\begin{array}{c}
d_{1}, d_{2}, \underset{3}{0}, \ldots, \underset{9}{0},-d_{3}, \underset{11}{0}, \ldots, \underset{20}{0},-d_{1} d_{3}, \underset{22}{0}, \ldots, \underset{27}{0}, d_{2} d_{3}, \underset{29}{0}, \\
-d_{2} d_{3}, \underset{31}{0}, \underset{32}{0}, d_{1} d_{2} d_{3}, \underset{34}{0},-d_{1} d_{2} d_{3}, \underset{36}{0}, \ldots, \underset{53}{0}
\end{array}\right) .
$$

For we have

$$
\begin{aligned}
& \mathfrak{n}_{\left(\gamma_{1342}, \gamma_{1432}\right)}^{4}\left(d_{1}, d_{2}, d_{3}, d_{4}, d_{5}\right) \\
& =\mathfrak{m}\left(\begin{array}{c}
d_{1}, d_{2}, d_{3}, d_{4}, \underset{5}{0}, \ldots, \underset{9}{0}, d_{3} d_{4}-d_{5}, \underset{11}{0}, \ldots, \underset{20}{0}, d_{1} d_{3} d_{4}-d_{1} d_{5}, \underset{22}{0}, \ldots, \underset{27}{0}, \underset{31}{0}, d_{2} \\
d_{2} d_{5}, \underset{29}{0}, d_{2} d_{3} d_{4}-d_{2} d_{5}, \underset{31}{0}, d_{1} d_{2} d_{5}, \underset{34}{0}, d_{1} d_{2} d_{3} d_{4}-d_{1} d_{2} d_{5}, \underset{36}{0}, \ldots, \underset{53}{0}
\end{array}\right) .
\end{aligned}
$$

For the sake of the completeness of our proof, we have provided the reason why (3.2) and (3.3) are derivable, but we will omit such reasoning from now on. (3.2) and (3.3) imply that

$$
\begin{aligned}
& \left(\left(\gamma_{1234}-\gamma_{12} \gamma_{1243}\right) \div\left(\gamma_{1342}-\gamma_{12}\right)\right)\left(d_{1}, d_{2}\right) \\
& =\mathfrak{m}\left(d_{1}, \underset{2}{0}, \ldots, \underset{25}{0},-d_{2}, \underset{27}{0},-d_{2}, \underset{29}{0}, d_{2},-d_{1} d_{2}, \underset{32}{0},-d_{1} d_{2}, \underset{34}{0}, d_{1} d_{2}, \underset{36}{0}, \ldots, \underset{53}{0}\right) \text {. }
\end{aligned}
$$

Since

$$
\begin{aligned}
& \gamma_{2341}\left(d_{1}, d_{2}, d_{3}, d_{4}\right) \\
& =\mathfrak{m}\left(\begin{array}{c}
d_{1}, d_{2}, d_{3}, d_{4}, d_{1} d_{2}, d_{1} d_{3}, d_{1} d_{4}, \underset{8}{0}, \ldots, \underset{12}{0}, d_{1} d_{2} d_{3}, \underset{14}{0}, \ldots, \underset{17}{0}, d_{1} d_{2} d_{4}, \underset{19}{0}, \ldots, \underset{22}{0}, \\
d_{1} d_{3} d_{4}, \underset{24}{0}, \ldots, \underset{38}{0}, d_{1} d_{2} d_{3} d_{4}, \underset{40}{0}, \ldots, \underset{53}{0}
\end{array}\right)
\end{aligned}
$$

and

$$
\begin{aligned}
& \gamma_{2431}\left(d_{1}, d_{2}, d_{3}, d_{4}\right) \\
& =\mathfrak{m}\left(\begin{array}{c}
d_{1}, d_{2}, d_{3}, d_{4}, d_{1} d_{2}, d_{1} d_{3}, d_{1} d_{4}, \underset{8}{0} \underset{9}{0}, d_{3} d_{4}, \underset{11}{0}, \underset{12}{0}, d_{1} d_{2} d_{3}, \underset{14}{0}, \ldots, \underset{17}{0}, d_{1} d_{2} d_{4}, \\
0, \ldots, \underset{24}{0}, d_{1} d_{3} d_{4}, d_{2} d_{3} d_{4}, \underset{27}{0}, \ldots, \underset{40}{0}, d_{1} d_{2} d_{3} d_{4}, \underset{42}{0}, \ldots, \underset{53}{0}
\end{array}\right),
\end{aligned}
$$

we have

$$
\begin{aligned}
& \left(\gamma_{2341}-\gamma_{2431}\right)\left(d_{1}, d_{2}, d_{3}\right) \\
& =\mathfrak{m}\left(\begin{array}{c}
d_{1}, d_{2}, \underset{3}{0}, \ldots,{ }_{9}^{0},-d_{3}, \underset{11}{0}, \ldots, \underset{22}{0}, d_{1} d_{3}, 0,-d_{1} d_{3},-d_{2} d_{3}, \\
0, \ldots, \underset{38}{0}, d_{1} d_{2} d_{3}, 0,-d_{1} d_{2} d_{3}, \underset{42}{0}, \ldots, 0 \\
27
\end{array}\right) .
\end{aligned}
$$

Since

$$
\begin{aligned}
& \gamma_{3421}\left(d_{1}, d_{2}, d_{3}, d_{4}\right) \\
& =\mathfrak{m}\left(\begin{array}{c}
d_{1}, d_{2}, d_{3}, d_{4}, d_{1} d_{2}, d_{1} d_{3}, d_{1} d_{4}, d_{2} d_{3}, d_{2} d_{4}, \underset{10}{0}, \ldots, \underset{14}{0}, d_{1} d_{2} d_{3}, \underset{16}{0}, \ldots, \underset{19}{0} \\
d_{1} d_{2} d_{4}, \underset{21}{0}, \underset{22}{0}, d_{1} d_{3} d_{4}, \underset{24}{0}, \ldots, \underset{27}{0}, d_{2} d_{3} d_{4}, \underset{29}{0}, \ldots, \underset{46}{0}, d_{1} d_{2} d_{3} d_{4}, \underset{48}{0}, \ldots, \underset{53}{0}
\end{array}\right)
\end{aligned}
$$

and

$\gamma_{4321}\left(d_{1}, d_{2}, d_{3}, d_{4}\right)$

$=\mathfrak{m}\left(\begin{array}{c}d_{1}, d_{2}, d_{3}, d_{4}, d_{1} d_{2}, d_{1} d_{3}, d_{1} d_{4}, d_{2} d_{3}, d_{2} d_{4}, d_{3} d_{4}, \underset{11}{0}, \ldots, \underset{14}{0}, d_{1} d_{2} d_{3}, \underset{16}{0}, \ldots, \underset{19}{0}, \\ d_{1} d_{2} d_{4}, \underset{21}{0}, \ldots, \underset{24}{0}, d_{1} d_{3} d_{4}, \underset{26}{0}, \ldots, \underset{29}{0}, d_{2} d_{3} d_{4}, \underset{31}{0}, \ldots, d_{1} d_{2} d_{3} d_{4}\end{array}\right)$, 
we have

$$
\begin{aligned}
& \left(\gamma_{3421}-\gamma_{12}\right)\left(d_{1}, d_{2}, d_{3}\right) \\
& =\mathfrak{m}\left(\begin{array}{c}
d_{1}, d_{2}, \underset{3}{0}, \ldots, \underset{9}{0},-d_{3}, \underset{11}{0}, \ldots, \underset{22}{0}, d_{1} d_{3}, \underset{24}{0},-d_{1} d_{3}, \underset{26}{0} \underset{27}{0}, d_{2} d_{3}, \underset{29}{0},-d_{2} d_{3}, \\
0, \ldots, \underset{46}{0}, d_{1} d_{2} d_{3}, \underset{48}{0}, \ldots, \underset{52}{0},-d_{1} d_{2} d_{3}
\end{array}\right) .
\end{aligned}
$$

(3.5) and (3.6) imply that

$$
\begin{aligned}
& \left(\left(\gamma_{2341} \frac{\cdot}{12} \gamma_{2431}\right)-\left(\gamma_{3421} \frac{\cdot}{12} \gamma_{4321}\right)\right)\left(d_{1}, d_{2}\right) \\
& =\mathfrak{m}\left(\begin{array}{c}
d_{1}, \underset{2}{0}, \ldots, \underset{25}{0},-d_{2}, \underset{27}{0},-d_{2}, \underset{29}{0}, d_{2}, \underset{31}{0}, \ldots, \underset{38}{0}, d_{1} d_{2}, \underset{40}{0}, \\
-d_{1} d_{2}, \underset{42}{0}, \ldots, \underset{46}{0},-d_{1} d_{2}, \underset{48}{0}, \ldots,{ }_{52}^{0}, d_{1} d_{2}
\end{array}\right) .
\end{aligned}
$$

(3.4) and (3.7) imply that

$$
\begin{aligned}
& \left.\left(\begin{array}{c}
\left(\left(\gamma_{1234}-\gamma_{1243}\right)-\left(\gamma_{1342}-\gamma_{1432}\right)\right)- \\
\left(\left(\gamma_{2341} \frac{-}{12} \gamma_{2431}\right)-\left(\gamma_{3421}-\gamma_{12}\right)\right.
\end{array}\right)\right)(d) \\
& =\mathfrak{m}(\underset{1}{0}, \ldots, \underset{30}{0},-d, \underset{32}{0},-d, \underset{34}{0}, d, \underset{36}{0}, \ldots, \underset{38}{0},-d, 0, d, \underset{42}{0}, \ldots, \underset{46}{0}, d, \underset{48}{0}, \ldots, \underset{52}{0},-d) .
\end{aligned}
$$

(2) Since

$$
\begin{aligned}
& \gamma_{1342}\left(d_{1}, d_{2}, d_{3}, d_{4}\right) \\
& =\mathfrak{m}\left(\begin{array}{c}
d_{1}, d_{2}, d_{3}, d_{4}, \underset{5}{0}, \ldots, \underset{7}{0}, d_{2} d_{3}, d_{2} d_{4}, \underset{10}{0}, d_{1} d_{2} d_{3}, \underset{12}{0}, \ldots, \underset{15}{0}, d_{1} d_{2} d_{4}, \underset{17}{0}, \ldots, \underset{27}{0}, \\
d_{2} d_{3} d_{4}, 0,0,0,0, d_{1} d_{2} d_{3} d_{4}, \underset{34}{0}, \ldots, \underset{53}{0}
\end{array}\right) \\
& \text { and } \\
& \gamma_{1324}\left(d_{1}, d_{2}, d_{3}, d_{4}\right) \\
& =\mathfrak{m}\left(\begin{array}{c}
d_{1}, d_{2}, d_{3}, d_{4}, \underset{5}{0}, \ldots, \underset{7}{0}, d_{2} d_{3}, \underset{9}{0} \underset{10}{0}, d_{1} d_{2} d_{3}, \underset{12}{0}, \ldots, \underset{26}{0}, d_{2} d_{3} d_{4}, \underset{28}{0}, \ldots, \underset{31}{0}, \\
d_{1} d_{2} d_{3} d_{4}, \underset{33}{0}, \ldots, \underset{53}{0}
\end{array}\right), \\
& \left(\gamma_{1342} \underset{13}{-} \gamma_{1324}\right)\left(d_{1}, d_{2}, d_{3}\right) \\
& =\mathfrak{m}\left(\begin{array}{c}
d_{1}, 0, d_{2}, \underset{4}{0}, \ldots, \underset{8}{0}, d_{3}, \underset{10}{0}, \ldots, \underset{15}{0}, d_{1} d_{3}, \underset{17}{0}, \ldots, \underset{26}{0},-d_{2} d_{3}, d_{2} d_{3}, \underset{29}{0}, \ldots, \underset{31}{0}, \\
-d_{1} d_{2} d_{3}, d_{1} d_{2} d_{3}, \underset{34}{0}, \ldots, \underset{53}{0}
\end{array}\right) .
\end{aligned}
$$

Since

$$
\begin{aligned}
& \gamma_{1423}\left(d_{1}, d_{2}, d_{3}, d_{4}\right) \\
& =\mathfrak{m}\left(\begin{array}{c}
d_{1}, d_{2}, d_{3}, d_{4}, \underset{5}{0}, \ldots, \underset{8}{0}, d_{2} d_{4}, d_{3} d_{4}, \underset{11}{0}, \ldots, \underset{15}{0}, d_{1} d_{2} d_{4}, \underset{17}{0}, \ldots, \underset{20}{0}, d_{1} d_{3} d_{4}, \\
0, \ldots, \underset{28}{0}, d_{2} d_{3} d_{4}, \underset{30}{0}, \ldots,{ }_{33}^{0}, d_{1} d_{2} d_{3} d_{4}, \underset{35}{0}, \ldots, \underset{53}{0}
\end{array}\right)
\end{aligned}
$$


and

$$
\begin{aligned}
& \gamma_{1243}\left(d_{1}, d_{2}, d_{3}, d_{4}\right) \\
& =\left(\begin{array}{c}
d_{1}, d_{2}, d_{3}, d_{4}, \underset{5}{0}, \ldots, \underset{9}{0}, d_{3} d_{4}, \underset{11}{0}, \ldots, \underset{20}{0}, d_{1} d_{3} d_{4}, \underset{22}{0}, \ldots, \underset{25}{0}, d_{2} d_{3} d_{4}, \underset{27}{0}, \ldots, \underset{30}{0}, \\
d_{1} d_{2} d_{3} d_{4}, \underset{32}{0}, \ldots, \underset{53}{0}
\end{array}\right),
\end{aligned}
$$

we have

$$
\begin{aligned}
& \left(\gamma_{1423}-\gamma_{1243}\right)\left(d_{1}, d_{2}, d_{3}\right) \\
& =\mathfrak{m}\left(\begin{array}{c}
d_{1}, 0, d_{2}, 0, \ldots, 0 \\
4
\end{array}, \begin{array}{c}
0, d_{3}, \underset{10}{0}, \ldots, \underset{15}{0}, d_{1} d_{3}, \underset{17}{0}, \ldots, \underset{25}{0},-d_{2} d_{3}, \underset{27}{0}, \underset{28}{0}, d_{2} d_{3}, \underset{30}{0}, \\
-d_{1} d_{2} d_{3}, \underset{32}{0}, \underset{33}{0}, d_{1} d_{2} d_{3}, \underset{35}{0}, \ldots, \underset{53}{0}
\end{array}\right) .
\end{aligned}
$$

(3.9) and (3.10) imply that

$$
\begin{aligned}
& \left(\left(\gamma_{1324} \frac{\cdot}{13} \gamma_{1342}\right) \div\left(\gamma_{1243} \frac{\dot{1}}{13} \gamma_{1423}\right)\right)\left(d_{1}, d_{2}\right) \\
& =\mathfrak{m}\left(d_{1}, \underset{2}{0}, \ldots, \underset{25}{0}, d_{2},-d_{2}, d_{2},-d_{2}, \underset{30}{0}, d_{1} d_{2},-d_{1} d_{2}, d_{1} d_{2},-d_{1} d_{2}, \underset{35}{0}, \ldots, \underset{53}{0}\right) \text {. }
\end{aligned}
$$

Since

$$
\begin{aligned}
& \gamma_{3421}\left(d_{1}, d_{2}, d_{3}, d_{4}\right) \\
& =\mathfrak{m}\left(\begin{array}{c}
d_{1}, d_{2}, d_{3}, d_{4}, d_{1} d_{2}, d_{1} d_{3}, d_{1} d_{4}, d_{2} d_{3}, d_{2} d_{4}, \underset{10}{0}, \ldots, \underset{14}{0}, d_{1} d_{2} d_{3}, \underset{16}{0}, \ldots, \underset{19}{0} d_{1} d_{2} d_{4}, 0,0, d_{1} d_{3} d_{4}, \underset{24}{0}, \ldots, \underset{27}{0}, d_{2} d_{3} d_{4}, \underset{29}{0}, \ldots, \underset{46}{0}, d_{1} d_{2} d_{3} d_{4}, \underset{48}{0}, \ldots, \underset{53}{0}
\end{array}\right)
\end{aligned}
$$

and

$$
\begin{aligned}
& \gamma_{3241}\left(d_{1}, d_{2}, d_{3}, d_{4}\right) \\
& =\mathfrak{m}\left(\begin{array}{c}
d_{1}, d_{2}, d_{3}, d_{4}, d_{1} d_{2}, d_{1} d_{3}, d_{1} d_{4}, d_{2} d_{3}, \underset{9}{0}, \ldots, \underset{14}{0}, d_{1} d_{2} d_{3}, \underset{16}{0}, \underset{17}{0}, d_{1} d_{2} d_{4}, \\
\underset{19}{0}, \ldots, \underset{22}{0}, d_{1} d_{3} d_{4}, \underset{24}{0}, \ldots, \underset{26}{0}, d_{2} d_{3} d_{4}, \underset{28}{0}, \ldots, \underset{44}{0}, d_{1} d_{2} d_{3} d_{4}, \underset{46}{0}, \ldots, \underset{53}{0}
\end{array}\right),
\end{aligned}
$$

we have

$$
\begin{aligned}
& \left(\gamma_{3421}-\gamma_{3241}\right)\left(d_{1}, d_{2}, d_{3}\right) \\
& =\mathfrak{m}\left(\begin{array}{c}
d_{1}, 0, d_{2}, \underset{4}{0}, \ldots, \underset{8}{0}, d_{3}, \underset{10}{0}, \ldots, \underset{17}{0},-d_{1} d_{3}, \underset{19}{0}, d_{1} d_{3}, \underset{21}{0}, \ldots, \underset{26}{0},-d_{2} d_{3}, \\
d_{2} d_{3}, \underset{29}{0}, \ldots, \underset{44}{0},-d_{1} d_{2} d_{3}, \underset{46}{0}, d_{1} d_{2} d_{3}, \underset{48}{0}, \ldots, \underset{53}{0}
\end{array}\right) .
\end{aligned}
$$

Since

$$
\begin{aligned}
& \gamma_{4231}\left(d_{1}, d_{2}, d_{3}, d_{4}\right) \\
& =\mathfrak{m}\left(\begin{array}{c}
d_{1}, d_{2}, d_{3}, d_{4}, d_{1} d_{2}, d_{1} d_{3}, d_{1} d_{4}, \underset{8}{0}, d_{2} d_{4}, d_{3} d_{4}, \underset{11}{0}, \underset{12}{0}, d_{1} d_{2} d_{3}, \underset{14}{0}, \ldots, \underset{19}{0}, \\
d_{1} d_{2} d_{4}, \underset{21}{0}, \ldots, \underset{24}{0}, d_{1} d_{3} d_{4}, \underset{26}{0}, \ldots, \underset{28}{0}, d_{2} d_{3} d_{4}, \underset{30}{0}, \ldots, d_{1} d_{2} d_{3} d_{4}, 0, \underset{53}{0}
\end{array}\right)
\end{aligned}
$$

$\gamma_{2431}\left(d_{1}, d_{2}, d_{3}, d_{4}\right)$ 


$$
=\mathfrak{m}\left(\begin{array}{c}
d_{1}, d_{2}, d_{3}, d_{4}, d_{1} d_{2}, d_{1} d_{3}, d_{1} d_{4}, \underset{8}{0}, \underset{9}{0}, d_{3} d_{4}, \underset{11}{0}, 0, d_{1} d_{2} d_{3}, \underset{14}{0}, \ldots, \underset{17}{0}, d_{1} d_{2} d_{4}, \\
0, \ldots, \underset{24}{0}, d_{1} d_{3} d_{4}, d_{2} d_{3} d_{4}, \underset{27}{0}, \ldots, \underset{40}{0}, d_{1} d_{2} d_{3} d_{4}, \underset{42}{0}, \ldots, \underset{53}{0}
\end{array}\right),
$$

we have

$$
\begin{aligned}
& \left(\gamma_{4231}-\gamma_{2431}\right)\left(d_{1}, d_{2}, d_{3}\right) \\
& =\mathfrak{m}\left(\begin{array}{c}
d_{1}, 0, d_{2}, \underset{2}{2}, \ldots, 0, d_{3}, 0, \ldots, \underset{10}{0}, 0-d_{1} d_{3}, \underset{19}{0}, d_{1} d_{3}, \underset{21}{0}, \ldots, \underset{25}{0},-d_{2} d_{3}, \\
0, \underset{28}{0}, d_{2} d_{3}, \underset{30}{0}, \ldots, \underset{40}{0},-d_{1} d_{2} d_{3}, \underset{42}{0}, \ldots, \underset{50}{0}, d_{1} d_{2} d_{3}, \underset{52}{0}, 0
\end{array}\right) .
\end{aligned}
$$

(3.12) and (3.13) imply that

$$
\begin{aligned}
& \left(\left(\gamma_{3421}-\gamma_{3241}\right)-\left(\gamma_{4231}-\gamma_{13}\right)\right) \\
& = \\
& =\mathfrak{m}\left(\begin{array}{c}
\left.d_{1},{ }_{1}, \ldots, d_{1}, d_{2}\right) \\
2, \underset{25}{0}, d_{2},-d_{2}, d_{2},-d_{2}, \underset{30}{0}, \ldots, \underset{40}{0}, d_{1} d_{2}, \underset{42}{0}, \ldots, \underset{44}{0}, \\
-d_{1} d_{2}, \underset{46}{0}, d_{1} d_{2}, \underset{48}{0}, \ldots, \underset{50}{0},-d_{1} d_{2}, \underset{52}{0}, 0
\end{array}\right) .
\end{aligned}
$$

(3.11) and (3.14) imply that

$$
\begin{aligned}
& \left(\begin{array}{c}
\left(\left(\gamma_{1324}-\gamma_{1342}\right)-\left(\gamma_{1243}-\gamma_{1423}\right)\right)- \\
\left(\left(\gamma_{3241}-\gamma_{13} \gamma_{3421}\right)-\left(\gamma_{2431}-\gamma_{13231}\right)\right)
\end{array}\right)(d) \\
& =\mathfrak{m}\left({ }_{1}^{0}, \ldots, \underset{30}{0}, d,-d, d,-d, \underset{35}{0}, \ldots, \underset{40}{0},-d, \underset{42}{0}, \ldots \underset{44}{0}, d, \underset{46}{0},-d, \underset{48}{0}, \ldots, \underset{50}{0}, d, \underset{52}{0}, 0\right) .
\end{aligned}
$$

(3) Since

$$
\begin{aligned}
& \gamma_{1423}\left(d_{1}, d_{2}, d_{3}, d_{4}\right) \\
& =\mathfrak{m}\left(\begin{array}{c}
d_{1}, d_{2}, d_{3}, d_{4}, \underset{5}{0}, \ldots, \underset{8}{0}, d_{2} d_{4}, d_{3} d_{4}, \underset{11}{0}, \ldots, \underset{15}{0}, d_{1} d_{2} d_{4}, \underset{17}{0}, \ldots, \underset{20}{0}, d_{1} d_{3} d_{4}, \\
{ }_{22}^{0}, \ldots, \underset{28}{0}, d_{2} d_{3} d_{4}, \underset{30}{0}, \ldots, \underset{33}{0}, d_{1} d_{2} d_{3} d_{4}, \underset{35}{0}, \ldots, \underset{53}{0}
\end{array}\right) \\
& \quad \text { and }
\end{aligned}
$$

$$
\begin{aligned}
& \gamma_{1432}\left(d_{1}, d_{2}, d_{3}, d_{4}\right) \\
& =\mathfrak{m}\left(\begin{array}{c}
d_{1}, d_{2}, d_{3}, d_{4}, \underset{5}{0}, \ldots, \underset{7}{0}, d_{2} d_{3}, d_{2} d_{4}, d_{3} d_{4}, d_{1} d_{2} d_{3}, \underset{12}{0}, \ldots, \underset{15}{0}, d_{1} d_{2} d_{4}, \underset{17}{0}, \ldots, \underset{20}{0}, \\
d_{1} d_{3} d_{4}, \underset{22}{0}, \ldots, \underset{29}{0}, d_{2} d_{3} d_{4}, \underset{31}{0}, \ldots, \underset{34}{0}, d_{1} d_{2} d_{3} d_{4}, \underset{36}{0}, \ldots, \underset{53}{0}
\end{array}\right),
\end{aligned}
$$

we have

$$
\begin{aligned}
& \left(\gamma_{1423} \frac{\dot{ }}{14} \gamma_{1432}\right)\left(d_{1}, d_{2}, d_{3}\right) \\
& =\mathfrak{m}\left(\begin{array}{c}
d_{1}, \underset{2}{0}, 0, d_{2}, \underset{5}{0}, \ldots, \underset{7}{0},-d_{3}, \underset{9}{0}, 0,-d_{1} d_{3}, \underset{12}{0}, \ldots, \underset{28}{0}, d_{2} d_{3},-d_{2} d_{3}, \\
0, \ldots,{ }_{33}^{0}, d_{1} d_{2} d_{3},-d_{1} d_{2} d_{3}, \underset{36}{0}, \ldots,{ }_{53}^{0}
\end{array}\right) .
\end{aligned}
$$

Since

$$
\gamma_{1234}\left(d_{1}, d_{2}, d_{3}, d_{4}\right)=\mathfrak{m}\left(d_{1}, d_{2}, d_{3}, d_{4}, 0, \ldots, 0\right)
$$


and

$$
\begin{aligned}
& \gamma_{1324}\left(d_{1}, d_{2}, d_{3}, d_{4}\right) \\
& =\mathfrak{m}\left(\begin{array}{c}
d_{1}, d_{2}, d_{3}, d_{4}, \underset{5}{0}, \ldots, \underset{7}{0}, d_{2} d_{3}, \underset{9}{0} \underset{10}{0}, d_{1} d_{2} d_{3}, \underset{12}{0}, \ldots, \underset{26}{0}, d_{2} d_{3} d_{4}, \underset{28}{0}, \ldots, \underset{30}{0}, d_{1} d_{2} d_{3} d_{4}, \underset{33}{0, \ldots,} \underset{53}{0}
\end{array}\right),
\end{aligned}
$$

we have

$$
\begin{aligned}
& \left(\gamma_{1234}-\gamma_{1324}\right)\left(d_{1}, d_{2}, d_{3}\right) \\
& =\mathfrak{m}\left(\begin{array}{c}
d_{1}, \underset{2}{0}, 0, d_{2}, \underset{5}{0}, \ldots, \underset{7}{0},-d_{3}, \underset{9}{0}, \underset{10}{0},-d_{1} d_{3}, \underset{12}{0}, \ldots, \underset{26}{0},-d_{2} d_{3}, \\
0, \ldots, \underset{31}{0},-d_{1} d_{2} d_{3}, \underset{33}{0}, \ldots, \underset{53}{0}
\end{array}\right) .
\end{aligned}
$$

(3.16) and (3.17) imply that

$$
\begin{aligned}
& \left(\left(\gamma_{1423} \underset{14}{-} \gamma_{1432}\right) \div\left(\gamma_{1234}-\gamma_{14} \gamma_{1324}\right)\right)\left(d_{1}, d_{2}\right) \\
& =\mathfrak{m}\left(d_{1}, \underset{2}{0}, \ldots, \underset{26}{0}, d_{2}, \underset{28}{0}, d_{2},-d_{2}, \underset{31}{0}, d_{1} d_{2}, \underset{33}{0}, d_{1} d_{2},-d_{1} d_{2}, \underset{36}{0}, \ldots, \underset{53}{0}\right) .
\end{aligned}
$$

Since

$$
\gamma_{4231}\left(d_{1}, d_{2}, d_{3}, d_{4}\right)
$$$$
=\mathfrak{m}\left(\begin{array}{c}
d_{1}, d_{2}, d_{3}, d_{4}, d_{1} d_{2}, d_{1} d_{3}, d_{1} d_{4}, \underset{8}{0}, d_{2} d_{4}, d_{3} d_{4}, \underset{11}{0}, \underset{12}{0}, d_{1} d_{2} d_{3}, \underset{14}{0}, \ldots, \underset{19}{0}, \\
d_{1} d_{2} d_{4}, \underset{21}{0}, \ldots, \underset{24}{0}, d_{1} d_{3} d_{4}, \underset{26}{0}, \ldots, \underset{28}{0}, d_{2} d_{3} d_{4}, \underset{30}{0}, \ldots, \underset{50}{0}, d_{1} d_{2} d_{3} d_{4}, \underset{52}{0}, \underset{53}{0}
\end{array}\right)
$$

and

$$
\begin{aligned}
& \gamma_{4321}\left(d_{1}, d_{2}, d_{3}, d_{4}\right) \\
& =\mathfrak{m}\left(\begin{array}{c}
d_{1}, d_{2}, d_{3}, d_{4}, d_{1} d_{2}, d_{1} d_{3}, d_{1} d_{4}, d_{2} d_{3}, d_{2} d_{4}, d_{3} d_{4}, \underset{11}{0}, \ldots, \underset{14}{0}, d_{1} d_{2} d_{3}, \underset{16}{0}, \ldots,{ }_{19}^{0}, \\
d_{1} d_{2} d_{4}, \underset{21}{0}, \ldots, \underset{24}{0}, d_{1} d_{3} d_{4}, \underset{26}{0}, \ldots, \underset{29}{0}, d_{2} d_{3} d_{4}, \underset{31}{0}, \ldots, d_{1} d_{2} d_{3} d_{4}
\end{array}\right), \\
& \text { we have } \\
& \quad\left(\begin{array}{c}
\gamma_{4231} \frac{-}{14} \gamma_{4321}
\end{array}\right)\left(d_{1}, d_{2}, d_{3}\right) \\
& \quad=\mathfrak{m}\left(\begin{array}{c}
d_{1}, \underset{2}{0}, \underset{3}{0}, d_{2}, \underset{5}{0}, \ldots, \underset{7}{0},-d_{3}, \underset{9}{0}, \ldots, \underset{12}{0}, d_{1} d_{3}, \underset{14}{0},-d_{1} d_{3}, \underset{16}{0}, \ldots, \underset{28}{0}, \\
d_{2} d_{3},-d_{2} d_{3}, \underset{31}{0}, \ldots, \underset{50}{0}, d_{1} d_{2} d_{3}, \underset{52}{0},-d_{1} d_{2} d_{3}
\end{array}\right) .
\end{aligned}
$$

\section{Since}

$$
\begin{aligned}
& \gamma_{2341}\left(d_{1}, d_{2}, d_{3}, d_{4}\right) \\
& =\mathfrak{m}\left(\begin{array}{c}
d_{1}, d_{2}, d_{3}, d_{4}, d_{1} d_{2}, d_{1} d_{3}, d_{1} d_{4}, \underset{8}{0}, \ldots, \underset{12}{0}, d_{1} d_{2} d_{3}, \underset{14}{0}, \ldots, \underset{17}{0}, d_{1} d_{2} d_{4}, \underset{19}{0}, \ldots, \underset{22}{0}, \\
d_{1} d_{3} d_{4}, \underset{24}{0}, \ldots, \underset{38}{0}, d_{1} d_{2} d_{3} d_{4}, \underset{40}{0}, \ldots, \underset{53}{0}
\end{array}\right) \\
& \text { and } \\
& \gamma_{3241}\left(d_{1}, d_{2}, d_{3}, d_{4}\right) \\
& =\mathfrak{m}\left(\begin{array}{c}
d_{1}, d_{2}, d_{3}, d_{4}, d_{1} d_{2}, d_{1} d_{3}, d_{1} d_{4}, d_{2} d_{3}, \underset{9}{0}, \ldots, \underset{14}{0}, d_{1} d_{2} d_{3}, \underset{16}{0}, \underset{17}{0}, d_{1} d_{2} d_{4}, \\
0, \ldots, \underset{22}{0}, d_{1} d_{3} d_{4}, \underset{24}{0}, \ldots, \underset{26}{0}, d_{2} d_{3} d_{4}, \underset{28}{0}, \ldots, \underset{44}{0}, d_{1} d_{2} d_{3} d_{4}, \underset{46}{0}, \ldots, \underset{53}{0}
\end{array}\right),
\end{aligned}
$$


we have

$$
\begin{aligned}
& \left(\gamma_{2341}-\gamma_{14} \gamma_{3241}\right)\left(d_{1}, d_{2}, d_{3}\right) \\
& =\mathfrak{m}\left(\begin{array}{c}
d_{1}, \underset{2}{0}, \underset{3}{0}, d_{2}, \underset{5}{0}, \ldots, \underset{7}{0},-d_{3}, \underset{9}{0}, \ldots, \underset{12}{0}, d_{1} d_{3}, \underset{14}{0},-d_{1} d_{3}, \underset{16}{0}, \ldots \underset{26}{0},-d_{2} d_{3}, \\
0, \ldots, \underset{38}{0}, d_{1} d_{2} d_{3}, \underset{40}{0}, \ldots, \underset{44}{0},-d_{1} d_{2} d_{3}, \underset{46}{0}, \ldots, \underset{53}{0}
\end{array}\right) .
\end{aligned}
$$

(3.19) and (3.20) imply that

$$
\begin{aligned}
& \left(\left(\gamma_{4231}-\gamma_{14}\right)-\left(\gamma_{2341}-\gamma_{3241}\right)\right)\left(d_{1}, d_{2}\right) \\
& =\mathfrak{m}\left(\begin{array}{c}
d_{1}, 0, \ldots, \underset{26}{0}, d_{2}, \underset{28}{0}, d_{2},-d_{2}, 0, \ldots, \underset{31}{0}, 0,-d_{1} d_{2}, \\
0, \ldots, \underset{44}{0}, d_{1} d_{2}, \underset{46}{0}, \ldots, \underset{50}{0}, d_{1} d_{2}, \underset{52}{0},-d_{1} d_{2}
\end{array}\right) .
\end{aligned}
$$

(3.18) and (3.21) imply that

$$
\begin{aligned}
& \left(\begin{array}{c}
\left(\left(\gamma_{1423}-\gamma_{1432}\right)-\left(\gamma_{1234}-\gamma_{1324}\right)\right)- \\
\left(\left(\gamma_{4231} \frac{-}{14} \gamma_{4321}\right)-\left(\gamma_{2341} \frac{\dot{1}}{14} \gamma_{3241}\right)\right)
\end{array}\right)(d) \\
& =\mathfrak{m}(\underset{1}{0}, \ldots, \underset{31}{0}, d, \underset{33}{0}, d,-d, \underset{36}{0}, \ldots, \underset{38}{0}, d, \underset{40}{0}, \ldots, \underset{44}{0},-d, \underset{46}{0}, \ldots, \underset{50}{0},-d, \underset{52}{0}, d) \text {. }
\end{aligned}
$$

(4) Since

$$
\begin{aligned}
& \gamma_{2143}\left(d_{1}, d_{2}, d_{3}, d_{4}\right) \\
& =\mathfrak{m}\left(\begin{array}{c}
d_{1}, d_{2}, d_{3}, d_{4}, d_{1} d_{2}, \underset{6}{0}, \ldots, \underset{9}{0}, d_{3} d_{4}, \underset{11}{0}, d_{1} d_{2} d_{3}, \underset{13}{0}, \ldots, \underset{16}{0}, d_{1} d_{2} d_{4}, \underset{18}{0}, \ldots, \underset{20}{0}, d_{1} d_{3} d_{4}, \underset{22}{0}, \ldots, \underset{25}{0}, d_{2} d_{3} d_{4}, \underset{27}{0}, \ldots, d_{1} d_{2} d_{3} d_{4}, \underset{38}{0}, \ldots, \underset{53}{0}
\end{array}\right) \\
& \quad \text { and } \\
& \qquad \gamma_{2134}\left(d_{1}, d_{2}, d_{3}, d_{4}\right) \\
& =\mathfrak{m}\left(\begin{array}{c}
d_{1}, d_{2}, d_{3}, d_{4}, d_{1} d_{2}, \underset{6}{0}, \ldots, \underset{11}{0}, d_{1} d_{2} d_{3}, \underset{13}{0}, \ldots, \underset{16}{0}, d_{1} d_{2} d_{4}, \\
0, \ldots, \underset{35}{0}, d_{1} d_{2} d_{3} d_{4}, \underset{37}{0}, \ldots, \underset{53}{0}
\end{array}\right),
\end{aligned}
$$

we have

$$
\begin{aligned}
& \left(\gamma_{2143} \frac{\dot{-}}{21} \gamma_{2134}\right)\left(d_{1}, d_{2}, d_{3}\right) \\
& =\mathfrak{m}\left(\begin{array}{c}
d_{2}, d_{1}, \underset{3}{0}, 0, d_{1} d_{2}, \underset{6}{0}, \ldots, \underset{9}{0}, d_{3}, \underset{11}{0}, \ldots, \underset{20}{0}, d_{2} d_{3}, \underset{22}{0}, \ldots, \underset{25}{0}, d_{1} d_{3}, \\
0, \ldots, \underset{35}{0},-d_{1} d_{2} d_{3}, d_{1} d_{2} d_{3}, \underset{38}{0}, \ldots, \underset{53}{0}
\end{array}\right) .
\end{aligned}
$$

Since

$$
\begin{aligned}
& \gamma_{2431}\left(d_{1}, d_{2}, d_{3}, d_{4}\right) \\
& =\mathfrak{m}\left(\begin{array}{c}
d_{1}, d_{2}, d_{3}, d_{4}, d_{1} d_{2}, d_{1} d_{3}, d_{1} d_{4}, \underset{8}{0} \underset{9}{0}, d_{3} d_{4}, \underset{11}{0}, \underset{12}{0}, d_{1} d_{2} d_{3}, \underset{14}{0}, \ldots, \underset{17}{0}, d_{1} d_{2} d_{4}, \\
0, \ldots, \underset{24}{0}, d_{1} d_{3} d_{4}, d_{2} d_{3} d_{4}, \underset{27}{0}, \ldots, \underset{40}{0}, d_{1} d_{2} d_{3} d_{4}, \underset{42}{0}, \ldots, \underset{53}{0}
\end{array}\right)
\end{aligned}
$$


and

$$
\begin{aligned}
& \gamma_{2341}\left(d_{1}, d_{2}, d_{3}, d_{4}\right) \\
& =\mathfrak{m}\left(\begin{array}{c}
d_{1}, d_{2}, d_{3}, d_{4}, d_{1} d_{2}, d_{1} d_{3}, d_{1} d_{4}, \underset{8}{0}, \ldots, \underset{12}{0}, d_{1} d_{2} d_{3}, \underset{14}{0}, \ldots, \underset{17}{0}, d_{1} d_{2} d_{4}, \underset{19}{0}, \ldots, \underset{22}{0}, \\
d_{1} d_{3} d_{4}, \underset{24}{0}, \ldots, \underset{38}{0}, d_{1} d_{2} d_{3} d_{4}, \underset{40}{0}, \ldots, \underset{53}{0}
\end{array}\right), \\
& \left(\gamma_{2431} \frac{\cdot}{21} \gamma_{2341}\right)\left(d_{1}, d_{2}, d_{3}\right) \\
& =\mathfrak{m}\left(\begin{array}{c}
d_{2}, d_{1}, \underset{3}{0}, \underset{4}{0}, d_{1} d_{2}, \underset{6}{0}, \ldots, \underset{9}{0}, d_{3}, \underset{11}{0}, \ldots, \underset{22}{0},-d_{2} d_{3}, \underset{24}{0}, d_{2} d_{3}, d_{1} d_{3}, \\
0, \ldots, \underset{38}{0},-d_{1} d_{2} d_{3}, \underset{40}{0}, d_{1} d_{2} d_{3}, \underset{42}{0}, \ldots, \underset{53}{0}
\end{array}\right) .
\end{aligned}
$$

(3.23) and (3.24) imply that

$$
\begin{aligned}
& \left(\left(\gamma_{2143}-\gamma_{2134}\right)-\left(\gamma_{2431}-\gamma_{21}\right)\right)\left(d_{1}, d_{2}\right) \\
& =\mathfrak{m}\left(\begin{array}{c}
0, d_{1}, 0, \ldots, \underset{30}{0}, d_{2}, \underset{22}{0}, d_{2}, \underset{24}{0},-d_{2}, \underset{26}{0}, \ldots, \underset{35}{0} \\
-d_{1} d_{2}, d_{1} d_{2}, \underset{38}{0}, d_{1} d_{2}, \underset{40}{0},-d_{1} d_{2}, \underset{42}{0}, \ldots, \underset{53}{0}
\end{array}\right) .
\end{aligned}
$$

Since

$$
\begin{aligned}
& \gamma_{1432}\left(d_{1}, d_{2}, d_{3}, d_{4}\right) \\
& =\mathfrak{m}\left(\begin{array}{c}
d_{1}, d_{2}, d_{3}, d_{4}, \underset{5}{0}, \ldots, \underset{7}{0}, d_{2} d_{3}, d_{2} d_{4}, d_{3} d_{4}, d_{1} d_{2} d_{3}, \underset{12}{0}, \ldots, \underset{15}{0}, d_{1} d_{2} d_{4}, \underset{17}{0}, \ldots, \underset{20}{0}, \\
d_{1} d_{3} d_{4}, \underset{22}{0}, \ldots, \underset{29}{0}, d_{2} d_{3} d_{4}, \underset{31}{0}, \ldots, \underset{34}{0}, d_{1} d_{2} d_{3} d_{4}, \underset{36}{0}, \ldots, \underset{53}{0}
\end{array}\right) \\
& =\mathfrak{m}\left(\begin{array}{c}
d_{1}, d_{2}, d_{3}, d_{4}, \underset{5}{0}, \ldots, \underset{7}{0}, d_{2} d_{3}, d_{2} d_{4}, \underset{10}{0}, d_{1} d_{2} d_{3}, \underset{12}{0}, \ldots, \underset{15}{0}, d_{1} d_{2} d_{4}, \underset{17}{0}, \ldots, \underset{27}{0}, \\
d_{2} d_{3} d_{4}, 0,0,0,0, d_{1} d_{2} d_{3} d_{4}, \underset{34}{0}, \ldots, \underset{53}{0}
\end{array}\right), \\
& \left(\gamma_{1432} \underset{21}{-} \gamma_{1342}\right)\left(d_{1}, d_{2}, d_{3}\right) \\
& =\mathfrak{m}\left(\begin{array}{c}
d_{2}, d_{1}, \underset{3}{0}, \underset{4}{0}, d_{1} d_{2}, \underset{6}{0}, \ldots, \underset{9}{0}, d_{3}, \underset{11}{0}, \ldots, \underset{20}{0}, d_{1} d_{3}, \underset{22}{0}, \ldots, \underset{27}{0},-d_{2} d_{3}, \underset{29}{0}, \\
d_{2} d_{3}, \underset{31}{0}, \underset{32}{0},-d_{1} d_{2} d_{3}, \underset{34}{0}, d_{1} d_{2} d_{3}, \underset{36}{0}, \ldots, \underset{53}{0}
\end{array}\right) .
\end{aligned}
$$

Since

$$
\begin{aligned}
& \gamma_{4312}\left(d_{1}, d_{2}, d_{3}, d_{4}\right) \\
& =\mathfrak{m}\left(\begin{array}{r}
d_{1}, d_{2}, d_{3}, d_{4}, \underset{5}{0}, d_{1} d_{3}, d_{1} d_{4}, d_{2} d_{3}, d_{2} d_{4}, d_{3} d_{4}, \underset{11}{0}, \ldots, \underset{13}{0}, d_{1} d_{2} d_{3}, \underset{15}{0}, \ldots, \underset{18}{0} d_{1} d_{2} d_{4}, \underset{20}{0}, \ldots, \underset{24}{0}, d_{1} d_{3} d_{4}, \underset{26}{0}, \ldots, \underset{29}{0}, d_{2} d_{3} d_{4}, \underset{31}{0}, \ldots, d_{1} d_{2} d_{3} d_{4}, \underset{53}{0}
\end{array}\right) \\
& \quad \text { and }
\end{aligned}
$$

$\gamma_{3412}\left(d_{1}, d_{2}, d_{3}, d_{4}\right)$ 
$=\left(\begin{array}{c}d_{1}, d_{2}, d_{3}, d_{4}, \underset{5}{0}, d_{1} d_{3}, d_{1} d_{4}, d_{2} d_{3}, d_{2} d_{4}, \underset{10}{0}, \ldots, \underset{13}{0}, d_{1} d_{2} d_{3}, \underset{15}{0}, \ldots, \underset{18}{0}, d_{1} d_{2} d_{4}, \\ 0, \ldots, \underset{22}{0}, d_{1} d_{3} d_{4}, \underset{24}{0}, \ldots, \underset{27}{0}, d_{2} d_{3} d_{4}, \underset{29}{0}, \ldots, d_{45} d_{2} d_{3} d_{4}, \underset{47}{0}, \ldots, \underset{53}{0}\end{array}\right)$,

we have

$$
\begin{aligned}
& \left(\gamma_{4312}-\gamma_{21}\right)\left(d_{1}, d_{2}, d_{3}\right) \\
& =\mathfrak{m}\left(\begin{array}{c}
d_{2}, d_{1}, \underset{3}{0}, \underset{4}{0}, d_{1} d_{2}, \underset{6}{0}, \ldots, \underset{9}{0}, d_{3}, \underset{11}{0}, \ldots, \underset{20}{0}, \underset{21}{0}, \underset{22}{0},-d_{2} d_{3}, \underset{24}{0}, d_{2} d_{3}, \underset{26}{0}, \underset{27}{0}, \\
-d_{1} d_{3}, \underset{29}{0}, d_{1} d_{3}, \underset{31}{0}, \ldots, \underset{45}{0},-d_{1} d_{2} d_{3}, \underset{47}{0}, \ldots, \underset{51}{0}, d_{1} d_{2} d_{3}, \underset{53}{0}
\end{array}\right) .
\end{aligned}
$$

(3.26) and (3.27) imply that

$$
\begin{aligned}
& \left(\left(\gamma_{1432}-\gamma_{21} \gamma_{1342}\right) \div\left(\gamma_{4312}-\gamma_{21} \gamma_{3412}\right)\right)\left(d_{1}, d_{2}\right) \\
& =\mathfrak{m}\left(\begin{array}{c}
0, d_{1}, \underset{3}{0}, \ldots, \underset{20}{0}, d_{2}, \underset{22}{0}, d_{2}, \underset{24}{0},-d_{2}, \underset{26}{0}, \ldots, \underset{32}{0},-d_{1} d_{2}, \underset{34}{0}, d_{1} d_{2}, \\
0, \ldots, \underset{45}{0}, d_{1} d_{2}, \underset{47}{0}, \ldots, \underset{51}{0},-d_{1} d_{2}, \underset{53}{0}
\end{array}\right) .
\end{aligned}
$$

(3.25) and (3.28) imply that

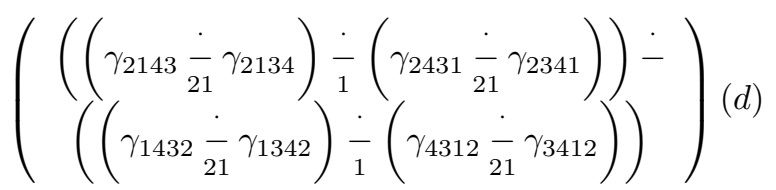

$$
\begin{aligned}
& =\mathfrak{m}(\underset{1}{0}, \ldots, \underset{32}{0}, d, \underset{34}{0},-d,-d, d, \underset{38}{0}, d, \underset{40}{0},-d, \underset{42}{0}, \ldots, \underset{45}{0},-d, \underset{47}{0}, \ldots, \underset{51}{0}, d, \underset{53}{0}) .
\end{aligned}
$$

(5) Since

$$
\begin{aligned}
& \gamma_{2314}\left(d_{1}, d_{2}, d_{3}, d_{4}\right) \\
& =\mathfrak{m}\left(\begin{array}{c}
d_{1}, d_{2}, d_{3}, d_{4}, d_{1} d_{2}, d_{1} d_{3}, \underset{7}{0}, \ldots, \underset{12}{0}, d_{1} d_{2} d_{3}, 0,0,0, \underset{1}{0}, d_{1} d_{2} d_{4}, \underset{18}{0}, \ldots, \underset{21}{0}, d_{1} d_{3} d_{4}, \\
0, \ldots, \underset{37}{0}, d_{1} d_{2} d_{3} d_{4}, \underset{39}{0}, \ldots, \underset{53}{0}
\end{array}\right) \\
& \quad \text { and }
\end{aligned}
$$

$\gamma_{2341}\left(d_{1}, d_{2}, d_{3}, d_{4}\right)$

$$
=\mathfrak{m}\left(\begin{array}{c}
d_{1}, d_{2}, d_{3}, d_{4}, d_{1} d_{2}, d_{1} d_{3}, d_{1} d_{4}, \underset{8}{0}, \ldots, \underset{12}{0}, d_{1} d_{2} d_{3}, \underset{14}{0}, \ldots, \underset{17}{0}, d_{1} d_{2} d_{4}, \underset{19}{0}, \ldots, \underset{22}{0}, \\
d_{1} d_{3} d_{4}, \underset{24}{0}, \ldots, \underset{38}{0}, d_{1} d_{2} d_{3} d_{4}, \underset{40}{0}, \ldots, \underset{53}{0}
\end{array}\right),
$$

we have

$$
\begin{aligned}
& \left(\gamma_{2314} \frac{\dot{2}}{23} \gamma_{2341}\right)\left(d_{1}, d_{2}, d_{3}\right) \\
& =\mathfrak{m}\left(\begin{array}{c}
0, d_{1}, d_{2}, \underset{4}{0}, \ldots, \underset{6}{0},-d_{3}, \underset{8}{0}, \ldots, \underset{16}{0}, d_{1} d_{3},-d_{1} d_{3}, \underset{19}{0}, \ldots, \underset{21}{0}, d_{2} d_{3},-d_{2} d_{3}, \\
0, \ldots,{ }_{37}^{0}, d_{1} d_{2} d_{3},-d_{1} d_{2} d_{3}, \underset{40}{0}, \ldots, \underset{53}{0}
\end{array}\right) .
\end{aligned}
$$

Since

$\gamma_{2143}\left(d_{1}, d_{2}, d_{3}, d_{4}\right)$ 


$$
=\mathfrak{m}\left(\begin{array}{c}
d_{1}, d_{2}, d_{3}, d_{4}, d_{1} d_{2}, \underset{6}{0}, \ldots, \underset{9}{0}, d_{3} d_{4}, 0, d_{1} d_{2} d_{3}, \underset{13}{0}, \ldots, \underset{16}{0}, d_{1} d_{2} d_{4}, 0,0,0, \\
d_{1} d_{3} d_{4}, \underset{22}{0}, \ldots, \underset{25}{0}, d_{2} d_{3} d_{4}, \underset{27}{0}, \ldots, \underset{36}{0}, d_{1} d_{2} d_{3} d_{4}, \underset{38}{0}, \ldots, \underset{53}{0}
\end{array}\right)
$$

and

$$
\begin{aligned}
& \gamma_{2413}\left(d_{1}, d_{2}, d_{3}, d_{4}\right) \\
& =\mathfrak{m}\left(\begin{array}{c}
d_{1}, d_{2}, d_{3}, d_{4}, d_{1} d_{2}, \underset{6}{0}, d_{1} d_{4}, \underset{8}{0} \underset{9}{0}, d_{3} d_{4}, \underset{11}{0}, d_{1} d_{2} d_{3}, \underset{13}{0}, \ldots, \underset{17}{0}, d_{1} d_{2} d_{4}, \\
\underset{19}{0}, \ldots, \underset{23}{0}, d_{1} d_{3} d_{4}, \underset{25}{0}, d_{2} d_{3} d_{4}, \underset{27}{0}, \ldots, \underset{39}{0}, d_{1} d_{2} d_{3} d_{4}, \underset{41}{0}, \ldots, \underset{53}{0}
\end{array}\right),
\end{aligned}
$$

we have

$$
\begin{aligned}
& \left(\gamma_{2143}-\gamma_{23}\right)\left(d_{1}, d_{2}, d_{3}\right) \\
& =\mathfrak{m}\left(\begin{array}{c}
0, d_{1}, d_{2}, 0, \ldots, \underset{6}{1},-d_{3}, \underset{8}{0}, \ldots, \underset{16}{0}, d_{1} d_{3},-d_{1} d_{3}, \underset{19}{0}, \underset{20}{0}, d_{2} d_{3}, \underset{22}{0}, \underset{23}{0}, \\
-d_{2} d_{3}, \underset{25}{0}, \ldots,{ }_{36}^{0}, d_{1} d_{2} d_{3}, \underset{38}{0}, \underset{39}{0},-d_{1} d_{2} d_{3}, \underset{41}{0}, \ldots, \underset{53}{0}
\end{array}\right) .
\end{aligned}
$$

(3.30) and (3.31) imply that

$$
\begin{aligned}
& \left(\left(\gamma_{2314} \frac{\dot{-}}{23} \gamma_{2341}\right)-\left(\gamma_{2143}-\gamma_{23}\right)\right)\left(d_{1}, d_{2}\right) \\
& =\mathfrak{m}\left(\begin{array}{cc}
0, d_{1}, 0, \ldots, \underset{20}{0},-d_{2}, d_{2},-d_{2}, d_{2}, \underset{25}{0}, \ldots, \underset{36}{0},-d_{1} d_{2}, d_{1} d_{2}, \\
-d_{1} d_{2}, d_{1} d_{2}, \underset{41}{0}, \ldots,{ }_{53}^{0}
\end{array}\right) .
\end{aligned}
$$

Since

$$
\begin{aligned}
& \gamma_{3142}\left(d_{1}, d_{2}, d_{3}, d_{4}\right) \\
& =\mathfrak{m}\left(\begin{array}{c}
d_{1}, d_{2}, d_{3}, d_{4}, \underset{5}{0}, d_{1} d_{3}, \underset{7}{0}, d_{2} d_{3}, d_{2} d_{4}, \underset{10}{0}, \ldots, \underset{13}{0}, d_{1} d_{2} d_{3}, 0, d_{1} d_{2} d_{4}, \underset{17}{0}, \ldots, \underset{21}{0}, \\
d_{1} d_{3} d_{4}, \underset{23}{0}, \ldots, \underset{27}{0}, d_{2} d_{3} d_{4}, \underset{29}{0}, \ldots, \underset{42}{0}, d_{1} d_{2} d_{3} d_{4}, \underset{44}{0}, \ldots, \underset{53}{0}
\end{array}\right)
\end{aligned}
$$

and

$$
\begin{aligned}
& \gamma_{3412}\left(d_{1}, d_{2}, d_{3}, d_{4}\right) \\
& =\mathfrak{m}\left(\begin{array}{r}
d_{1}, d_{2}, d_{3}, d_{4}, \underset{5}{0}, d_{1} d_{3}, d_{1} d_{4}, d_{2} d_{3}, d_{2} d_{4}, \underset{10}{0}, \ldots, \underset{13}{0}, d_{1} d_{2} d_{3}, \underset{15}{0}, \ldots, \underset{18}{0}, d_{1} d_{2} d_{4}, \\
0, \ldots, \underset{22}{0}, d_{1} d_{3} d_{4}, \underset{24}{0}, \ldots, \underset{27}{0}, d_{2} d_{3} d_{4}, \underset{29}{0}, \ldots, d_{1} d_{2} d_{3} d_{4}, \underset{47}{0}, \ldots, \underset{53}{0}
\end{array}\right), \\
& \quad\left(\begin{array}{c}
\gamma_{3142}-\gamma_{3412} \\
23
\end{array}\right)\left(d_{1}, d_{2}, d_{3}\right) \\
& =\mathfrak{m}\left(\begin{array}{c}
0, d_{1}, d_{2}, d_{4}, \ldots, \underset{6}{1},-d_{3}, \underset{8}{0}, \ldots, \underset{15}{0}, d_{1} d_{3}, \underset{17}{0}, \underset{18}{0},-d_{1} d_{3}, \underset{20}{0}, \underset{21}{0}, d_{2} d_{3}, \\
-d_{2} d_{3}, \underset{24}{0}, \ldots, \underset{42}{0}, d_{1} d_{2} d_{3}, \underset{44}{0}, \underset{45}{0},-d_{1} d_{2} d_{3}, \underset{47}{0}, \ldots, \underset{53}{0}
\end{array}\right) .
\end{aligned}
$$

Since

$\gamma_{1432}\left(d_{1}, d_{2}, d_{3}, d_{4}\right)$

$$
=\mathfrak{m}\left(\begin{array}{c}
d_{1}, d_{2}, d_{3}, d_{4}, \underset{5}{0}, \ldots, \underset{7}{0}, d_{2} d_{3}, d_{2} d_{4}, d_{3} d_{4}, d_{1} d_{2} d_{3}, \underset{12}{0}, \ldots, \underset{15}{0}, d_{1} d_{2} d_{4}, \underset{17}{0}, \ldots, \underset{20}{0}, \\
d_{1} d_{3} d_{4}, \underset{22}{0}, \ldots, \underset{29}{0}, d_{2} d_{3} d_{4}, \underset{31}{0}, \ldots, \underset{34}{0}, d_{1} d_{2} d_{3} d_{4}, \underset{36}{0}, \ldots, \underset{53}{0}
\end{array}\right)
$$


and

$$
\begin{aligned}
& \gamma_{4132}\left(d_{1}, d_{2}, d_{3}, d_{4}\right) \\
& =\mathfrak{m}\left(\begin{array}{c}
d_{1}, d_{2}, d_{3}, d_{4}, \underset{5}{0}, \underset{6}{0}, d_{1} d_{4}, d_{2} d_{3}, d_{2} d_{4}, d_{3} d_{4}, d_{1} d_{2} d_{3}, \underset{12}{0}, \ldots, \underset{18}{0}, d_{1} d_{2} d_{4}, \\
0, \ldots, \underset{23}{0}, d_{1} d_{3} d_{4}, \underset{25}{0}, \ldots, \underset{29}{0}, d_{2} d_{3} d_{4}, \underset{31}{0}, \ldots, \underset{48}{0}, d_{1} d_{2} d_{3} d_{4}, \underset{50}{0}, \ldots, \underset{53}{0}
\end{array}\right),
\end{aligned}
$$

we have

$$
\begin{aligned}
& \left(\gamma_{1432}-\gamma_{23}\right)\left(d_{1}, d_{2}, d_{3}\right) \\
& =\mathfrak{m}\left(\begin{array}{c}
0, d_{1}, d_{2}, \underset{4}{1}, \ldots, \underset{6}{0},-d_{3}, \underset{8}{0}, \ldots, \underset{15}{0}, d_{1} d_{3}, \underset{17}{0}, \underset{18}{0},-d_{1} d_{3}, \underset{20}{0}, d_{2} d_{3}, \underset{22}{0}, \underset{23}{0} \\
-d_{2} d_{3}, \underset{25}{0}, \ldots, \underset{34}{0}, d_{1} d_{2} d_{3}, \underset{36}{0}, \ldots, \underset{48}{0},-d_{1} d_{2} d_{3}, \underset{50}{0}, \ldots, \underset{53}{0}
\end{array}\right) .
\end{aligned}
$$

(3.33) and (3.34) imply that

$$
\begin{aligned}
& \left(\left(\gamma_{3142}-\gamma_{23} \gamma_{3412}\right)-\left(\gamma_{1432}-\gamma_{23}\right)\right)\left(d_{1}, d_{2}\right) \\
& =\mathfrak{m}\left(\begin{array}{c}
0, d_{1},{ }_{3}^{0}, \ldots, \underset{20}{1},-d_{2}, d_{2},-d_{2}, d_{2}, \underset{25}{0}, \ldots, \underset{34}{0},-d_{1} d_{2}, \underset{36}{0}, \ldots,{ }_{42}^{0} \\
d_{1} d_{2}, \underset{44}{0}, \underset{45}{0},-d_{1} d_{2}, \underset{47}{0}, \underset{48}{0}, d_{1} d_{2}, \underset{50}{0}, \ldots,{ }_{53}^{0}
\end{array}\right) .
\end{aligned}
$$

(3.32) and (3.35) imply that

$$
\begin{aligned}
& \left.\left(\begin{array}{c}
\left(\left(\gamma_{2314}-\gamma_{23} \gamma_{2341}\right)-\left(\gamma_{2143}-\gamma_{2413}\right)\right)- \\
\left(\left(\gamma_{3142}-\gamma_{23} \gamma_{3412}\right)-\left(\gamma_{1432}-\gamma_{23}\right)\right.
\end{array}\right)\right)(d) \\
& =\mathfrak{m}(\underset{1}{0}, \ldots, \underset{34}{0}, d, \underset{36}{0},-d, d,-d, d, \underset{41}{0}, \underset{42}{0},-d, \underset{44}{0}, \underset{45}{0}, d, \underset{47}{0}, \underset{48}{0},-d, \underset{50}{0}, \ldots, \underset{53}{0}) .
\end{aligned}
$$

(6) Since

$$
\begin{aligned}
& \gamma_{2431}\left(d_{1}, d_{2}, d_{3}, d_{4}\right) \\
& =\mathfrak{m}\left(\begin{array}{c}
d_{1}, d_{2}, d_{3}, d_{4}, d_{1} d_{2}, d_{1} d_{3}, d_{1} d_{4}, \underset{8}{0}, \underset{9}{0}, d_{3} d_{4}, \underset{11}{0}, \underset{12}{0}, d_{1} d_{2} d_{3}, \underset{14}{0}, \ldots, \underset{17}{0}, d_{1} d_{2} d_{4}, \\
0, \ldots, \underset{24}{0}, d_{1} d_{3} d_{4}, d_{2} d_{3} d_{4}, \underset{27}{0}, \ldots, \underset{40}{0}, d_{1} d_{2} d_{3} d_{4}, \underset{42}{0}, \ldots, \underset{53}{0}
\end{array}\right)
\end{aligned}
$$

and

$$
\begin{aligned}
& \gamma_{2413}\left(d_{1}, d_{2}, d_{3}, d_{4}\right) \\
& =\mathfrak{m}\left(\begin{array}{c}
d_{1}, d_{2}, d_{3}, d_{4}, d_{1} d_{2}, \underset{6}{0}, d_{1} d_{4}, \underset{8}{0} \underset{9}{0}, d_{3} d_{4}, \underset{11}{0}, d_{1} d_{2} d_{3}, \underset{13}{0}, \ldots, \underset{17}{0}, d_{1} d_{2} d_{4}, \\
0, \ldots, \underset{23}{0}, d_{1} d_{3} d_{4}, 0, d_{2} d_{3} d_{4}, \underset{27}{0}, \ldots, \underset{39}{0}, d_{1} d_{2} d_{3} d_{4}, \underset{41}{0}, \ldots, \underset{53}{0}
\end{array}\right),
\end{aligned}
$$

we have

$$
\begin{aligned}
& \left(\gamma_{2431} \frac{\cdot}{24} \gamma_{2413}\right)\left(d_{1}, d_{2}, d_{3}\right) \\
& =\mathfrak{m}\left(\begin{array}{c}
0, d_{1}, 0, d_{2}, \underset{5}{1}, d_{3}, \underset{7}{0}, \ldots, \underset{11}{0},-d_{1} d_{3}, d_{1} d_{3}, \underset{14}{0}, \ldots, \underset{23}{0}, \\
-d_{2} d_{3}, d_{2} d_{3}, \underset{26}{0}, \ldots, \underset{39}{0},-d_{1} d_{2} d_{3}, d_{1} d_{2} d_{3}, \underset{42}{0}, \ldots, \underset{53}{0}
\end{array}\right) .
\end{aligned}
$$

Since

$\gamma_{2314}\left(d_{1}, d_{2}, d_{3}, d_{4}\right)$ 
$=\mathfrak{m}\left(\begin{array}{c}d_{1}, d_{2}, d_{3}, d_{4}, d_{1} d_{2}, d_{1} d_{3}, \underset{7}{0}, \ldots, \underset{12}{0}, d_{1} d_{2} d_{3}, 0,0,0, d_{1} d_{2} d_{4}, \underset{18}{0}, \ldots, \underset{21}{0}, d_{1} d_{3} d_{4}, \\ 0, \ldots, \underset{37}{0}, d_{1} d_{2} d_{3} d_{4}, \underset{39}{0}, \ldots, \underset{53}{0}\end{array}\right)$

and

$$
\begin{aligned}
& \gamma_{2134}\left(d_{1}, d_{2}, d_{3}, d_{4}\right) \\
& =\left(\begin{array}{c}
d_{1}, d_{2}, d_{3}, d_{4}, d_{1} d_{2}, \underset{6}{0}, \ldots, \underset{11}{0}, d_{1} d_{2} d_{3}, \underset{13}{0}, \ldots, \underset{16}{0}, d_{1} d_{2} d_{4}, \\
0, \ldots, \underset{35}{0}, d_{1} d_{2} d_{3} d_{4}, \underset{37}{0}, \ldots, \underset{53}{0}
\end{array}\right),
\end{aligned}
$$

we have

$$
\begin{aligned}
& \left(\gamma_{2314}-\gamma_{2134}\right)\left(d_{1}, d_{2}, d_{3}\right) \\
& =\mathfrak{m}\left(\begin{array}{c}
0, d_{1}, \underset{1}{1}, d_{2}, \underset{5}{0}, d_{3}, \underset{7}{0}, \ldots, \underset{11}{0},-d_{1} d_{3}, d_{1} d_{3}, \underset{14}{0}, \ldots, \underset{21}{0}, d_{2} d_{3}, \\
0, \ldots, \underset{35}{0},-d_{1} d_{2} d_{3}, \underset{37}{0}, d_{1} d_{2} d_{3}, \underset{39}{0}, \ldots, \underset{53}{0}
\end{array}\right) .
\end{aligned}
$$

(3.37) and (3.38) imply that

$$
\begin{aligned}
& \left(\left(\gamma_{2431} \frac{\dot{2}}{24} \gamma_{2413}\right) \div\left(\gamma_{2314} \frac{\cdot}{24} \gamma_{2134}\right)\right)\left(d_{1}, d_{2}\right) \\
& =\mathfrak{m}\left(\begin{array}{c}
0, d_{1}, \underset{3}{0}, \ldots, \underset{21}{0},-d_{2}, \underset{23}{0},-d_{2}, d_{2}, \underset{26}{0}, \ldots, \underset{35}{0}, d_{1} d_{2}, \underset{37}{0},-d_{1} d_{2}, \\
0,-d_{1} d_{2}, d_{1} d_{2}, \underset{42}{0}, \ldots, \underset{53}{0}
\end{array}\right) .
\end{aligned}
$$

Since

$$
\begin{aligned}
& \gamma_{4312}\left(d_{1}, d_{2}, d_{3}, d_{4}\right) \\
& =\mathfrak{m}\left(\begin{array}{c}
d_{1}, d_{2}, d_{3}, d_{4}, \underset{5}{0}, d_{1} d_{3}, d_{1} d_{4}, d_{2} d_{3}, d_{2} d_{4}, d_{3} d_{4}, \underset{11}{0}, \ldots, \underset{13}{0}, d_{1} d_{2} d_{3}, \underset{15}{0}, \ldots, \underset{18}{0}, \underset{20}{0} d_{1} d_{2} d_{4}, \underset{24}{0}, \ldots, d_{2}, d_{1} d_{3} d_{4}, \underset{26}{0}, \ldots, \underset{29}{0}, d_{2} d_{3} d_{4}, \underset{31}{0}, \ldots, d_{2} d_{3} d_{4}, \underset{53}{0}
\end{array}\right) \\
& \gamma_{4132}\left(d_{1}, d_{2}, d_{3}, d_{4}\right) \\
& =\mathfrak{m}\left(\begin{array}{c}
d_{1}, d_{2}, d_{3}, d_{4}, \underset{5}{0}, \underset{6}{0}, d_{1} d_{4}, d_{2} d_{3}, d_{2} d_{4}, d_{3} d_{4}, d_{1} d_{2} d_{3}, \underset{12}{0}, \ldots, \underset{18}{0}, d_{1} d_{2} d_{4}, \\
0, \ldots, \underset{23}{0}, d_{1} d_{3} d_{4}, \underset{25}{0}, \ldots, \underset{29}{0}, d_{2} d_{3} d_{4}, \underset{31}{0}, \ldots, \underset{48}{0}, d_{1} d_{2} d_{3} d_{4}, \underset{50}{0}, \ldots, \underset{53}{0}
\end{array}\right),
\end{aligned}
$$

we have

$$
\begin{aligned}
& \left(\gamma_{4312}-\gamma_{24}\right)\left(d_{1}, d_{2}, d_{3}\right) \\
& =\mathfrak{m}\left(\begin{array}{c}
0, d_{1}, \underset{3}{1}, d_{2},{ }_{5}, d_{3}, \underset{7}{0}, \ldots, \underset{10}{0},-d_{1} d_{3}, \underset{12}{0}, \underset{13}{0}, d_{1} d_{3}, \underset{15}{0}, \ldots, \underset{23}{0}, \\
-d_{2} d_{3}, d_{2} d_{3}, \underset{26}{0}, \ldots, \underset{48}{0},-d_{1} d_{2} d_{3}, \underset{50}{0}, 0, d_{1} d_{2} d_{3}, \underset{53}{0}
\end{array}\right) .
\end{aligned}
$$

Since

$$
\begin{aligned}
& \gamma_{3142}\left(d_{1}, d_{2}, d_{3}, d_{4}\right) \\
& =\mathfrak{m}\left(\begin{array}{c}
d_{1}, d_{2}, d_{3}, d_{4}, \underset{5}{0}, d_{1} d_{3}, \underset{7}{0}, d_{2} d_{3}, d_{2} d_{4}, \underset{10}{0}, \ldots, \underset{13}{0}, d_{1} d_{2} d_{3}, 0, d_{1} d_{2} d_{4}, \underset{17}{0}, \ldots, \underset{21}{0}, \\
d_{1} d_{3} d_{4}, \underset{23}{0}, \ldots, \underset{27}{0}, d_{2} d_{3} d_{4}, \underset{29}{0}, \ldots, \underset{42}{0}, d_{1} d_{2} d_{3} d_{4}, \underset{44}{0}, \ldots, \underset{53}{0}
\end{array}\right)
\end{aligned}
$$


and

$$
\begin{aligned}
& \gamma_{1342}\left(d_{1}, d_{2}, d_{3}, d_{4}\right) \\
& =\mathfrak{m}\left(\begin{array}{c}
d_{1}, d_{2}, d_{3}, d_{4}, \underset{5}{0}, \ldots, \underset{7}{0}, d_{2} d_{3}, d_{2} d_{4}, \underset{10}{0}, d_{1} d_{2} d_{3}, \underset{12}{0}, \ldots, \underset{15}{0}, d_{1} d_{2} d_{4}, \underset{17}{0}, \ldots, \underset{27}{0}, \\
d_{2} d_{3} d_{4}, 0,0,0,0, d_{1} d_{2} d_{3} d_{4}, \underset{34}{0}, \ldots, \underset{53}{0}
\end{array}\right), \\
& \left(\gamma_{3142} \frac{\dot{2}}{24} \gamma_{1342}\right)\left(d_{1}, d_{2}, d_{3}\right) \\
& =\mathfrak{m}\left(\begin{array}{c}
0, d_{1}, \underset{1}{0}, d_{2}, \underset{5}{0}, d_{3}, \underset{7}{0}, \ldots, \underset{10}{0},-d_{1} d_{3}, \underset{12}{0}, \underset{13}{0}, d_{1} d_{3}, \underset{15}{0}, \ldots, \underset{21}{0}, d_{2} d_{3}, \\
0, \ldots, \underset{32}{0},-d_{1} d_{2} d_{3}, \underset{34}{0}, \ldots, \underset{42}{0}, d_{1} d_{2} d_{3}, \underset{44}{0}, \ldots, \underset{53}{0}
\end{array}\right) .
\end{aligned}
$$

(3.40) and (3.41) imply that

$$
\begin{aligned}
& \left(\left(\gamma_{4312}-\gamma_{24}\right) \cdot\left(\gamma_{3142}-\gamma_{1342}\right)\right)\left(d_{1}, d_{2}\right) \\
& =\mathfrak{m}\left(\begin{array}{c}
0, d_{1}, \underset{1}{1}, \ldots, \underset{21}{0},-d_{2}, \underset{23}{0},-d_{2}, d_{2}, \underset{26}{0}, \ldots, \underset{32}{0}, d_{1} d_{2}, \underset{34}{0}, \ldots, \underset{42}{0}, \\
-d_{1} d_{2}, \underset{44}{0}, \ldots, \underset{48}{0},-d_{1} d_{2}, \underset{50}{0}, \underset{51}{0}, d_{1} d_{2}, \underset{53}{0}
\end{array}\right) .
\end{aligned}
$$

(3.39) and (3.42) imply that

$$
\begin{aligned}
& \left.\left(\begin{array}{c}
\left(\left(\gamma_{2431}-\gamma_{24}\right)-\left(\gamma_{2314}-\gamma_{2134}\right)\right)- \\
\left(\left(\gamma_{4312} \frac{-}{24} \gamma_{4132}\right)-\left(\gamma_{3142}-\gamma_{24}\right)\right.
\end{array}\right)\right)(d) \\
& =\mathfrak{m}(\underset{1}{0}, \ldots, \underset{32}{0},-d, \underset{34}{0}, \underset{35}{0}, d, \underset{37}{0},-d, \underset{39}{0},-d, d, \underset{42}{0}, d, \underset{44}{0}, \ldots, \underset{48}{0}, d, \underset{50}{0}, \underset{51}{0},-d, \underset{53}{0}) .
\end{aligned}
$$

(7) Since

$$
\begin{aligned}
& \gamma_{3124}\left(d_{1}, d_{2}, d_{3}, d_{4}\right) \\
& =\mathfrak{m}\left(\begin{array}{c}
d_{1}, d_{2}, d_{3}, d_{4}, \underset{5}{0}, d_{1} d_{3}, \underset{7}{0}, d_{2} d_{3}, \underset{9}{0}, \ldots, \underset{13}{0}, d_{1} d_{2} d_{3}, \underset{15}{0}, \ldots, \underset{21}{0}, d_{1} d_{3} d_{4}, \underset{23}{0}, \ldots, \underset{26}{0}, \\
d_{2} d_{3} d_{4}, \underset{28}{0}, \ldots, \underset{41}{0}, d_{1} d_{2} d_{3} d_{4}, \underset{43}{0}, \ldots, \underset{53}{0}
\end{array}\right)
\end{aligned}
$$

$$
\begin{aligned}
& \gamma_{3142}\left(d_{1}, d_{2}, d_{3}, d_{4}\right) \\
& =\mathfrak{m}\left(\begin{array}{c}
d_{1}, d_{2}, d_{3}, d_{4}, \underset{5}{0}, d_{1} d_{3}, \underset{7}{0}, d_{2} d_{3}, d_{2} d_{4}, \underset{10}{0}, \ldots, \underset{13}{0}, d_{1} d_{2} d_{3}, 0, d_{1} d_{2} d_{4}, \underset{17}{0}, \ldots, \underset{21}{0}, \\
d_{1} d_{3} d_{4}, \underset{23}{0}, \ldots, \underset{27}{0}, d_{2} d_{3} d_{4}, \underset{29}{0}, \ldots, \underset{42}{0}, d_{1} d_{2} d_{3} d_{4}, \underset{44}{0}, \ldots, \underset{53}{0}
\end{array}\right),
\end{aligned}
$$

we have

$$
\begin{aligned}
& \left(\gamma_{3124} \frac{\dot{-}}{31} \gamma_{3142}\right)\left(d_{1}, d_{2}, d_{3}\right) \\
& =\mathfrak{m}\left(\begin{array}{c}
d_{2}, \underset{2}{0}, d_{1}, \underset{4}{0}, \ldots, \underset{8}{0},-d_{3}, \underset{10}{0}, \ldots, \underset{15}{0},-d_{2} d_{3}, \underset{17}{0}, \ldots, \underset{26}{0}, d_{1} d_{3},-d_{1} d_{3}, \\
0, \ldots, \underset{41}{0}, d_{1} d_{2} d_{3},-d_{1} d_{2} d_{3}, \underset{44}{0}, \ldots,{ }_{53}^{0}
\end{array}\right) .
\end{aligned}
$$

Since

$\gamma_{3241}\left(d_{1}, d_{2}, d_{3}, d_{4}\right)$ 
$=\mathfrak{m}\left(\begin{array}{c}d_{1}, d_{2}, d_{3}, d_{4}, d_{1} d_{2}, d_{1} d_{3}, d_{1} d_{4}, d_{2} d_{3}, \underset{9}{0}, \ldots, \underset{14}{0}, d_{1} d_{2} d_{3}, \underset{16}{0}, \underset{17}{0}, d_{1} d_{2} d_{4}, \\ 0, \ldots, \underset{22}{0}, d_{1} d_{3} d_{4}, \underset{24}{0}, \ldots, \underset{26}{0}, d_{2} d_{3} d_{4}, \underset{28}{0}, \ldots, \underset{44}{0}, d_{1} d_{2} d_{3} d_{4}, \underset{46}{0}, \ldots, \underset{53}{0}\end{array}\right)$

and

$\gamma_{3421}\left(d_{1}, d_{2}, d_{3}, d_{4}\right)$

$=\mathfrak{m}\left(\begin{array}{c}d_{1}, d_{2}, d_{3}, d_{4}, d_{1} d_{2}, d_{1} d_{3}, d_{1} d_{4}, d_{2} d_{3}, d_{2} d_{4}, \underset{10}{0}, \ldots, \underset{14}{0}, d_{1} d_{2} d_{3}, \underset{16}{0}, \ldots, \underset{19}{0}, \\ d_{1} d_{2} d_{4}, \underset{21}{0}, 0, d_{1} d_{3} d_{4}, \underset{24}{0}, \ldots, \underset{27}{0}, d_{2} d_{3} d_{4}, \underset{29}{0}, \ldots, \underset{46}{0}, d_{1} d_{2} d_{3} d_{4}, \underset{48}{0}, \ldots,{ }_{53}^{0}\end{array}\right)$,

we have

$\left(\gamma_{3241} \frac{\cdot}{31} \gamma_{3421}\right)\left(d_{1}, d_{2}, d_{3}\right)$

$=\mathfrak{m}\left(\begin{array}{c}d_{2}, \underset{2}{0}, d_{1}, \underset{4}{0}, \ldots, \underset{8}{0},-d_{3}, \underset{10}{0}, \ldots, \underset{17}{0}, d_{2} d_{3}, \underset{19}{0},-d_{2} d_{3}, \underset{21}{0}, \ldots, \underset{26}{0}, \\ d_{1} d_{3},-d_{1} d_{3}, \underset{29}{0}, \ldots, \underset{44}{0}, d_{1} d_{2} d_{3}, \underset{46}{0},-d_{1} d_{2} d_{3}, \underset{48}{0}, \ldots, \underset{53}{0}\end{array}\right)$.

(3.44) and (3.45) imply that

$$
\begin{aligned}
& \left(\left(\gamma_{3124} \frac{-}{31} \gamma_{3142}\right)-\left(\gamma_{3241}-\gamma_{3421}\right)\right)\left(d_{1}, d_{2}\right) \\
& =\mathfrak{m}\left(\begin{array}{c}
0,0, d_{1}, 0, \ldots, \underset{15}{0},-d_{2}, 0,-d_{2}, \underset{19}{2}, d_{2}, \underset{21}{0}, \ldots, \underset{41}{0}, \\
d_{1} d_{2},-d_{1} d_{2}, 0,-d_{14} d_{2}, \underset{46}{0}, d_{1} d_{2}, \underset{48}{0}, \ldots,{ }_{53}^{0}
\end{array}\right) .
\end{aligned}
$$

Since

$\gamma_{1243}\left(d_{1}, d_{2}, d_{3}, d_{4}\right)$

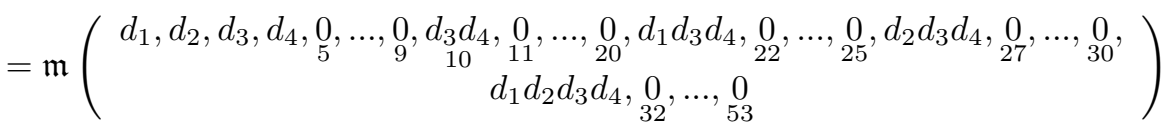

and

$\gamma_{1423}\left(d_{1}, d_{2}, d_{3}, d_{4}\right)$

$=\mathfrak{m}\left(\begin{array}{c}d_{1}, d_{2}, d_{3}, d_{4}, \underset{5}{0}, \ldots, \underset{8}{0}, d_{2} d_{4}, d_{3} d_{4}, \underset{11}{0}, \ldots, \underset{15}{0}, d_{1} d_{2} d_{4}, \underset{17}{0}, \ldots, \underset{20}{0}, d_{1} d_{3} d_{4}, \\ 02, \ldots, \underset{28}{0}, d_{2} d_{3} d_{4}, \underset{30}{0}, \ldots, \underset{33}{0}, d_{1} d_{2} d_{3} d_{4}, \underset{35}{0}, \ldots, \underset{53}{0}\end{array}\right)$,

we have

$$
\begin{aligned}
& \left(\gamma_{1243} \frac{\dot{-}}{31} \gamma_{1423}\right)\left(d_{1}, d_{2}, d_{3}\right) \\
& =\mathfrak{m}\left(\begin{array}{c}
d_{2}, \underset{2}{0}, d_{1}, \underset{4}{0}, \ldots, \underset{8}{0},-d_{3}, \underset{10}{0}, \ldots, \underset{15}{0},-d_{2} d_{3}, \underset{17}{0}, \ldots, \underset{25}{0}, d_{1} d_{3}, \\
27, \underset{28}{0},-d_{1} d_{3}, \underset{30}{0}, d_{1} d_{2} d_{3}, \underset{32}{0}, \underset{33}{0},-d_{1} d_{2} d_{3}, \underset{35}{0}, \ldots, \underset{53}{0}
\end{array}\right) .
\end{aligned}
$$

Since

$$
\begin{aligned}
& \gamma_{2413}\left(d_{1}, d_{2}, d_{3}, d_{4}\right) \\
& =\mathfrak{m}\left(\begin{array}{c}
d_{1}, d_{2}, d_{3}, d_{4}, d_{1} d_{2}, \underset{6}{0}, d_{1} d_{4}, \underset{8}{0}, \underset{9}{0}, d_{3} d_{4}, \underset{11}{0}, d_{1} d_{2} d_{3}, \underset{13}{0}, \ldots, \underset{17}{0}, d_{1} d_{2} d_{4}, \\
0, \ldots, \underset{23}{0}, d_{1} d_{3} d_{4}, 0, d_{2} d_{3} d_{4}, \underset{27}{0}, \ldots, \underset{39}{0}, d_{1} d_{2} d_{3} d_{4}, \underset{41}{0}, \ldots, \underset{53}{0}
\end{array}\right)
\end{aligned}
$$


and

$\gamma_{4213}\left(d_{1}, d_{2}, d_{3}, d_{4}\right)$
$=\mathfrak{m}\left(\begin{array}{c}d_{1}, d_{2}, d_{3}, d_{4}, d_{1} d_{2}, \underset{6}{0}, d_{1} d_{4}, \underset{8}{0}, d_{2} d_{4}, d_{3} d_{4}, \underset{11}{0}, d_{1} d_{2} d_{3}, \underset{13}{0}, \ldots, \underset{19}{0}, d_{1} d_{2} d_{4}, \\ 0, \ldots, \underset{23}{0}, d_{1} d_{3} d_{4}, \underset{25}{0}, \ldots, \underset{28}{0}, d_{2} d_{3} d_{4}, \underset{30}{0}, \ldots, \underset{49}{0}, d_{1} d_{2} d_{3} d_{4}, \underset{51}{0}, \ldots, \underset{53}{0}\end{array}\right)$,

we have

$$
\begin{aligned}
& \left(\gamma_{2413} \underset{31}{-} \gamma_{4213}\right)\left(d_{1}, d_{2}, d_{3}\right) \\
& =\mathfrak{m}\left(\begin{array}{c}
d_{2}, \underset{2}{0}, d_{1}, \underset{4}{0}, \ldots, \underset{8}{0},-d_{3}, \underset{10}{0}, \ldots, \underset{17}{0}, d_{2} d_{3}, \underset{19}{0},-d_{2} d_{3}, \underset{21}{0}, \ldots, \underset{25}{0}, d_{1} d_{3}, \\
0, \underset{28}{0},-d_{1} d_{3}, \underset{30}{0}, \ldots, \underset{39}{0}, d_{1} d_{2} d_{3}, \underset{41}{0}, \ldots, \underset{49}{0},-d_{1} d_{2} d_{3}, \underset{51}{0}, \ldots, \underset{53}{0}
\end{array}\right) .
\end{aligned}
$$

(3.47) and (3.48) imply that

$$
\begin{aligned}
& \left(\left(\gamma_{1243} \frac{\cdot}{31} \gamma_{1423}\right)-\left(\gamma_{2413}-\gamma_{4213}\right)\right)\left(d_{1}, d_{2}\right) \\
& =\mathfrak{m}\left(\begin{array}{c}
0,0, d_{1}, \underset{1}{1}, \ldots, \underset{15}{0},-d_{2}, \underset{17}{0},-d_{2}, \underset{19}{0}, d_{2}, \underset{21}{0}, \ldots, \underset{30}{0}, d_{1} d_{2}, \underset{32}{0}, \underset{33}{0},-d_{1} d_{2}, \\
0, \ldots, \underset{39}{0},-d_{1} d_{2}, \underset{41}{0}, \ldots, \underset{49}{0}, d_{1} d_{2}, \underset{51}{0}, \ldots, \underset{53}{0}
\end{array}\right) .
\end{aligned}
$$

(3.46) and (3.49) imply that

$$
\begin{aligned}
& \left(\begin{array}{c}
\left(\left(\gamma_{3124} \frac{\dot{-}}{31} \gamma_{3142}\right)-\left(\gamma_{3241}-\gamma_{3421}\right)\right)- \\
\left(\left(\gamma_{1243} \frac{\dot{-}}{31} \gamma_{1423}\right)-\left(\gamma_{2413} \frac{\dot{-}}{31} \gamma_{4213}\right)\right)
\end{array}\right)(d) \\
& =\mathfrak{m}\left(\begin{array}{c}
0, \ldots, \underset{30}{0},-d, \underset{32}{0}, \underset{33}{0}, d, \underset{35}{0}, \ldots, \underset{39}{0}, d, \underset{41}{0}, d,-d, \underset{44}{0},-d, \underset{46}{0}, d, \\
0,0,-d,{ }_{51}^{0}, \ldots, 0 \\
53
\end{array}\right) .
\end{aligned}
$$

(8) Since

$$
\begin{aligned}
& \gamma_{3241}\left(d_{1}, d_{2}, d_{3}, d_{4}\right) \\
& =\mathfrak{m}\left(\begin{array}{c}
d_{1}, d_{2}, d_{3}, d_{4}, d_{1} d_{2}, d_{1} d_{3}, d_{1} d_{4}, d_{2} d_{3}, \underset{9}{0}, \ldots, \underset{14}{0}, d_{1} d_{2} d_{3}, \underset{16}{0}, \underset{17}{0}, d_{1} d_{2} d_{4}, \\
0, \ldots, \underset{22}{0}, d_{1} d_{3} d_{4}, \underset{24}{0}, \ldots, \underset{26}{0}, d_{2} d_{3} d_{4}, \underset{28}{0}, \ldots, \underset{44}{0}, d_{1} d_{2} d_{3} d_{4}, \underset{46}{0}, \ldots, \underset{53}{0}
\end{array}\right) \\
& \text { and } \\
& \gamma_{3214}\left(d_{1}, d_{2}, d_{3}, d_{4}\right) \\
& =\mathfrak{m}\left(\begin{array}{c}
d_{1}, d_{2}, d_{3}, d_{4}, d_{1} d_{2}, d_{1} d_{3}, 0, d_{2} d_{3}, \underset{9}{0}, \ldots, \underset{14}{0}, d_{1} d_{2} d_{3}, 0, d_{1} d_{2} d_{4}, \underset{18}{0}, \ldots, \underset{21}{0}, \\
d_{1} d_{3} d_{4}, \underset{23}{0}, \ldots, \underset{26}{0}, d_{2} d_{3} d_{4}, \underset{28}{0}, \ldots, \underset{43}{0}, d_{1} d_{2} d_{3} d_{4}, \underset{45}{0}, \ldots, \underset{53}{0}
\end{array}\right), \\
& \text { we have } \\
& \left(\gamma_{3241}-\gamma_{32} \gamma_{3214}\right)\left(d_{1}, d_{2}, d_{3}\right) \\
& =\mathfrak{m}\left(\begin{array}{c}
0, d_{2}, d_{1}, \underset{4}{0}, \ldots, \underset{6}{0}, d_{3}, \underset{8}{0}, \ldots, \underset{16}{0},-d_{2} d_{3}, d_{2} d_{3}, \underset{19}{0}, \ldots, \underset{21}{0},-d_{1} d_{3}, d_{1} d_{3}, \\
0, \ldots, \underset{43}{0},-d_{1} d_{2} d_{3}, d_{1} d_{2} d_{3}, \underset{46}{0}, \ldots, \underset{53}{0}
\end{array}\right) .
\end{aligned}
$$


Since

$\gamma_{3412}\left(d_{1}, d_{2}, d_{3}, d_{4}\right)$
$=\mathfrak{m}\left(\begin{array}{c}d_{1}, d_{2}, d_{3}, d_{4}, \underset{5}{0}, d_{1} d_{3}, d_{1} d_{4}, d_{2} d_{3}, d_{2} d_{4}, \underset{10}{0}, \ldots, \underset{13}{0}, d_{1} d_{2} d_{3}, \underset{15}{0}, \ldots, \underset{18}{0}, d_{1} d_{2} d_{4}, \\ 0, \ldots,{ }_{22}^{0}, d_{1} d_{3} d_{4}, \underset{24}{0}, \ldots, \underset{27}{0}, d_{2} d_{3} d_{4}, \underset{29}{0}, \ldots, \underset{45}{0}, d_{1} d_{2} d_{3} d_{4}, \underset{47}{0}, \ldots, \underset{53}{0}\end{array}\right)$

and

$\gamma_{3142}\left(d_{1}, d_{2}, d_{3}, d_{4}\right)$

$=\mathfrak{m}\left(\begin{array}{c}d_{1}, d_{2}, d_{3}, d_{4}, \underset{5}{0}, d_{1} d_{3}, \underset{7}{0}, d_{2} d_{3}, d_{2} d_{4}, \underset{10}{0}, \ldots, \underset{13}{0}, d_{1} d_{2} d_{3}, 0, d_{1} d_{2} d_{4}, \underset{17}{0}, \ldots, \underset{21}{0}, \\ d_{1} d_{3} d_{4}, \underset{23}{0}, \ldots, \underset{27}{0}, d_{2} d_{3} d_{4}, \underset{29}{0}, \ldots, \underset{42}{0}, d_{1} d_{2} d_{3} d_{4}, \underset{44}{0}, \ldots, \underset{53}{0}\end{array}\right)$,

we have

$\left(\gamma_{3412} \frac{-}{32} \gamma_{3142}\right)\left(d_{1}, d_{2}, d_{3}\right)$

$=\mathfrak{m}\left(\begin{array}{c}0, d_{2}, d_{1}, \underset{4}{1}, \ldots, \underset{6}{0}, d_{3}, \underset{8}{0}, \ldots, \underset{15}{0},-d_{2} d_{3}, \underset{17}{0}, \underset{18}{0}, d_{2} d_{3}, \underset{20}{0}, \underset{21}{0}, \\ -d_{1} d_{3}, d_{1} d_{3}, \underset{24}{0}, \ldots, \underset{42}{0},-d_{1} d_{2} d_{3}, \underset{44}{0}, \underset{45}{0}, d_{1} d_{2} d_{3}, \underset{47}{0}, \ldots, \underset{53}{0}\end{array}\right)$.

(3.51) and (3.52) imply that

$\left(\left(\gamma_{3241} \frac{-}{32} \gamma_{3214}\right)-\left(\gamma_{3412}-\gamma_{32}\right)\right)\left(d_{1}, d_{2}\right)$
$=\mathfrak{m}\left(\begin{array}{c}0,0, d_{1}, \underset{4}{1}, \ldots, \underset{15}{0}, d_{2},-d_{2}, d_{2},-d_{2}, \underset{20}{0}, \ldots, \underset{42}{0}, d_{1} d_{2},-d_{1} d_{2}, \\ d_{1} d_{2},-d_{1} d_{2}, \underset{47}{0}, \ldots, \underset{53}{0}\end{array}\right)$.

Since

$\gamma_{2413}\left(d_{1}, d_{2}, d_{3}, d_{4}\right)$

$=\mathfrak{m}\left(\begin{array}{c}d_{1}, d_{2}, d_{3}, d_{4}, d_{1} d_{2}, \underset{6}{0}, d_{1} d_{4}, \underset{8}{0} \underset{9}{0}, d_{3} d_{4}, \underset{11}{0}, d_{1} d_{2} d_{3}, \underset{13}{0}, \ldots, \underset{17}{0}, d_{1} d_{2} d_{4}, \\ 0, \ldots, \underset{23}{0}, d_{1} d_{3} d_{4}, 0, d_{2} d_{3} d_{4}, \underset{27}{0}, \ldots, \underset{39}{0}, d_{1} d_{2} d_{3} d_{4}, \underset{41}{0}, \ldots, \underset{53}{0}\end{array}\right)$

and

$\gamma_{2143}\left(d_{1}, d_{2}, d_{3}, d_{4}\right)$

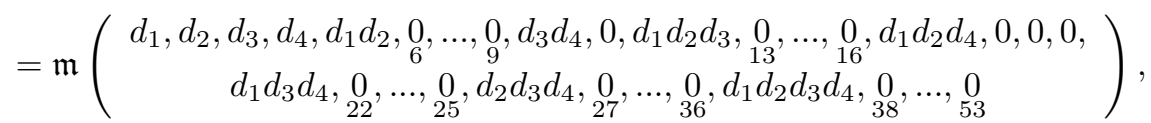

we have

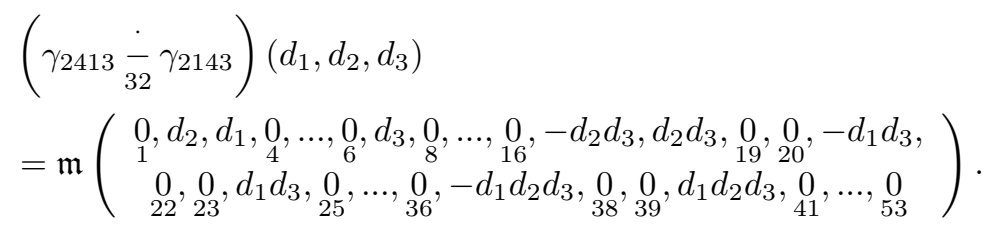

Since

$\gamma_{4123}\left(d_{1}, d_{2}, d_{3}, d_{4}\right)$ 


$$
\begin{aligned}
& =\mathfrak{m}\left(\begin{array}{c}
d_{1}, d_{2}, d_{3}, d_{4}, \underset{5}{0}, \underset{6}{0}, d_{1} d_{4}, \underset{8}{0}, d_{2} d_{4}, d_{3} d_{4}, \underset{11}{0}, \ldots, \underset{18}{0}, d_{1} d_{2} d_{4}, \underset{20}{0}, \ldots, \underset{23}{0}, d_{1} d_{3} d_{4}, \\
0, \ldots, \underset{28}{0}, d_{2} d_{3} d_{4}, \underset{30}{0}, \ldots, \underset{47}{0}, d_{1} d_{2} d_{3} d_{4}, \underset{49}{0}, \ldots, \underset{53}{0}
\end{array}\right) \\
& =\mathfrak{m}\left(\begin{array}{c}
d_{1}, d_{2}, d_{3}, d_{4}, \underset{5}{0}, \ldots, \underset{8}{0}, d_{2} d_{4}, d_{3} d_{4}, \underset{11}{0}, \ldots, \underset{15}{0}, d_{1} d_{2} d_{4}, \underset{17}{0}, \ldots, \underset{20}{0}, d_{1} d_{3} d_{4}, \\
0, \ldots, \underset{28}{0}, d_{2} d_{3} d_{4}, \underset{30}{0}, \ldots,{ }_{33}^{0}, d_{1} d_{2} d_{3} d_{4}, \underset{35}{0}, \ldots, \underbrace{0}_{53}
\end{array}\right),
\end{aligned}
$$

we have

$$
\begin{aligned}
& \left(\gamma_{4123} \frac{\cdot}{32} \gamma_{1423}\right)\left(d_{1}, d_{2}, d_{3}\right) \\
& =\mathfrak{m}\left(\begin{array}{c}
0, d_{2}, d_{1}, \underset{4}{1}, \ldots, \underset{6}{0}, d_{3}, \underset{8}{0}, \ldots, \underset{15}{0},-d_{2} d_{3}, \underset{17}{0}, \underset{18}{0}, d_{2} d_{3}, \underset{20}{0},-d_{1} d_{3}, \\
0,0, d_{1} d_{3}, \underset{25}{0}, \ldots, \underset{33}{0},-d_{1} d_{2} d_{3}, \underset{35}{0}, \ldots, \underset{47}{0}, d_{1} d_{2} d_{3}, \underset{49}{0}, \ldots,{ }_{53}^{0}
\end{array}\right) .
\end{aligned}
$$

(3.54) and (3.55) imply that

$$
\begin{aligned}
& \left(\left(\gamma_{2413} \frac{-}{32} \gamma_{2143}\right)-\left(\gamma_{4123} \frac{-}{32} \gamma_{1423}\right)\right)\left(d_{1}, d_{2}\right) \\
& =\mathfrak{m}\left(\begin{array}{c}
0,0, d_{1},{ }_{2}^{0}, \ldots, \underset{15}{0}, d_{2},-d_{2}, d_{2},-d_{2}, \underset{20}{0}, \ldots,{ }_{33}^{0}, d_{1} d_{2}, \underset{35}{0}, 0,-d_{16} d_{2}, \\
0,0, d_{1} d_{2}, \underset{41}{0}, \ldots, \underset{47}{0},-d_{1} d_{2}, \underset{49}{0}, \ldots,{ }_{53}^{0}
\end{array}\right) .
\end{aligned}
$$

(3.53) and (3.56) imply that

$$
\begin{aligned}
& \left(\begin{array}{c}
\left(\left(\gamma_{3241} \frac{\dot{-}}{32} \gamma_{3214}\right)-\left(\gamma_{3412}-\gamma_{3242}\right)\right)- \\
\left(\left(\gamma_{2413}-\gamma_{2143}\right)-\left(\gamma_{4123}-\gamma_{1423}\right)\right)
\end{array}\right)(d) \\
& =\mathfrak{m}(\underset{1}{0}, \ldots, \underset{33}{0},-d, \underset{35}{0}, \underset{36}{0}, d, \underset{38}{0} \underset{39}{0},-d, \underset{41}{0}, \underset{42}{0}, d,-d, d,-d, \underset{47}{0}, d, \underset{49}{0}, \ldots, \underset{53}{0}) .
\end{aligned}
$$

(9) Since

$$
\begin{aligned}
& \gamma_{3412}\left(d_{1}, d_{2}, d_{3}, d_{4}\right) \\
& =\mathfrak{m}\left(\begin{array}{c}
d_{1}, d_{2}, d_{3}, d_{4}, \underset{5}{0}, d_{1} d_{3}, d_{1} d_{4}, d_{2} d_{3}, d_{2} d_{4}, \underset{10}{0}, \ldots, \underset{13}{0}, d_{1} d_{2} d_{3}, \underset{15}{0}, \ldots, \underset{18}{0}, d_{1} d_{2} d_{4}, \\
0, \ldots, \underset{22}{0}, d_{1} d_{3} d_{4}, \underset{24}{0}, \ldots, \underset{27}{0}, d_{2} d_{3} d_{4}, \underset{29}{0}, \ldots, \underset{45}{0}, d_{1} d_{2} d_{3} d_{4}, \underset{47}{0}, \ldots, \underset{53}{0}
\end{array}\right) \\
& \text { and } \\
& \gamma_{3421}\left(d_{1}, d_{2}, d_{3}, d_{4}\right) \\
& =\mathfrak{m}\left(\begin{array}{c}
d_{1}, d_{2}, d_{3}, d_{4}, d_{1} d_{2}, d_{1} d_{3}, d_{1} d_{4}, d_{2} d_{3}, d_{2} d_{4}, \underset{10}{0}, \ldots, \underset{14}{0}, d_{1} d_{2} d_{3}, \underset{16}{0}, \ldots, \underset{19}{0}, \\
d_{1} d_{2} d_{4}, \underset{21}{0}, \underset{22}{0}, d_{1} d_{3} d_{4}, \underset{24}{0}, \ldots, \underset{27}{0}, d_{2} d_{3} d_{4}, \underset{29}{0}, \ldots, \underset{46}{0}, d_{1} d_{2} d_{3} d_{4}, \underset{48}{0}, \ldots, \underset{53}{0}
\end{array}\right), \\
& \text { we have } \\
& \left(\gamma_{3412} \underset{34}{-} \gamma_{3421}\right)\left(d_{1}, d_{2}, d_{3}\right) \\
& =\mathfrak{m}\left(\begin{array}{c}
0, \underset{2}{0}, d_{1}, d_{2},-d_{3}, \underset{6}{0}, \ldots, \underset{13}{0}, d_{1} d_{3},-d_{1} d_{3}, \underset{16}{0}, \ldots, \underset{18}{0}, d_{2} d_{3},-d_{2} d_{3}, \\
0, \ldots, \underset{45}{0}, d_{1} d_{2} d_{3},-d_{1} d_{2} d_{3}, \underset{48}{0}, \ldots, \underset{53}{0}
\end{array}\right) .
\end{aligned}
$$


Since

$$
\begin{aligned}
& \gamma_{3124}\left(d_{1}, d_{2}, d_{3}, d_{4}\right) \\
& =\mathfrak{m}\left(\begin{array}{c}
d_{1}, d_{2}, d_{3}, d_{4}, \underset{5}{0}, d_{1} d_{3}, \underset{7}{0}, d_{2} d_{3}, \underset{9}{0}, \ldots, \underset{13}{0}, d_{1} d_{2} d_{3}, \underset{15}{0}, \ldots, \underset{21}{0}, d_{1} d_{3} d_{4}, \underset{23}{0}, \ldots, \underset{26}{0}, \\
d_{2} d_{3} d_{4}, \underset{28}{0}, \ldots, \underset{41}{0}, d_{1} d_{2} d_{3} d_{4}, \underset{43}{0}, \ldots, \underset{53}{0}
\end{array}\right) \\
& \gamma_{3214}\left(d_{1}, d_{2}, d_{3}, d_{4}\right) \\
& =\mathfrak{m}\left(\begin{array}{c}
d_{1}, d_{2}, d_{3}, d_{4}, d_{1} d_{2}, d_{1} d_{3}, \underset{7}{0}, d_{2} d_{3}, \underset{9}{0}, \ldots, \underset{14}{0}, d_{1} d_{2} d_{3}, 0, d_{1} d_{2} d_{4}, \underset{18}{0}, \ldots, \underset{21}{0}, \\
d_{1} d_{3} d_{4}, \underset{23}{0}, \ldots, \underset{26}{0}, d_{2} d_{3} d_{4}, \underset{28}{0}, \ldots, \underset{43}{0}, d_{1} d_{2} d_{3} d_{4}, \underset{45}{0}, \ldots, \underset{53}{0}
\end{array}\right), \\
& \left(\gamma_{3124} \frac{\cdot}{34} \gamma_{3214}\right)\left(d_{1}, d_{2}, d_{3}\right) \\
& =\mathfrak{m}\left(\begin{array}{c}
0, \underset{2}{0}, d_{1}, d_{2},-d_{3}, \underset{6}{0}, \ldots, \underset{13}{0}, d_{1} d_{3},-d_{1} d_{3}, \underset{16}{0},-d_{2} d_{3}, \underset{18}{0}, \ldots, \underset{41}{0}, \\
d_{1} d_{2} d_{3}, \underset{43}{0},-d_{1} d_{2} d_{3}, \underset{45}{0}, \ldots, \underset{53}{0}
\end{array}\right) .
\end{aligned}
$$

(3.58) and (3.59) imply that

$$
\begin{aligned}
& \left(\left(\gamma_{3412} \frac{\dot{-}}{34} \gamma_{3421}\right)-\left(\gamma_{3124} \frac{\dot{-}}{34} \gamma_{3214}\right)\right)\left(d_{1}, d_{2}\right) \\
& =\mathfrak{m}\left(\begin{array}{c}
0,0, d_{1},{ }_{2}^{0}, \ldots, \underset{16}{0}, d_{2}, \underset{18}{0}, d_{2},-d_{2}, \underset{21}{0}, \ldots{ }_{41}^{0},-d_{1} d_{2}, \underset{43}{0}, d_{1} d_{2}, \\
0, d_{1} d_{2},-d_{1} d_{2}, \underset{48}{0}, \ldots, \underset{53}{0}
\end{array}\right) .
\end{aligned}
$$

Since

$$
\begin{aligned}
& \gamma_{4123}\left(d_{1}, d_{2}, d_{3}, d_{4}\right) \\
& =\mathfrak{m}\left(\begin{array}{c}
d_{1}, d_{2}, d_{3}, d_{4}, \underset{5}{0}, \underset{6}{0}, d_{1} d_{4}, \underset{8}{0}, d_{2} d_{4}, d_{3} d_{4}, \underset{11}{0}, \ldots, \underset{18}{0}, d_{1} d_{2} d_{4}, \underset{20}{0}, \ldots, \underset{23}{0}, d_{1} d_{3} d_{4}, \\
0, \ldots, \underset{28}{0}, d_{2} d_{3} d_{4}, \underset{30}{0}, \ldots, \underset{47}{0}, d_{1} d_{2} d_{3} d_{4}, \underset{49}{0}, \ldots,{ }_{53}^{0}
\end{array}\right) \\
& =\mathfrak{m}\left(\begin{array}{c}
d_{1}, d_{2}, d_{3}, d_{4}, d_{1} d_{2}, 0, d_{1} d_{4}, 0, d_{2} d_{4}, d_{3} d_{4}, 0, d_{1} d_{2} d_{3}, \underset{13}{0}, \ldots, \underset{19}{0}, d_{1} d_{2} d_{4}, \\
0, \ldots, \underset{23}{0}, d_{1} d_{3} d_{4}, \underset{25}{0}, \ldots, \underset{28}{0}, d_{2} d_{3} d_{4}, \underset{30}{0}, \ldots, \underset{49}{0}, d_{1} d_{2} d_{3} d_{4}, \underset{51}{0}, \ldots, \underset{53}{0}
\end{array}\right),
\end{aligned}
$$

we have

$$
\begin{aligned}
& \left(\gamma_{4123} \frac{\dot{-}}{34} \gamma_{4213}\right)\left(d_{1}, d_{2}, d_{3}\right) \\
& =\mathfrak{m}\left(\begin{array}{c}
0, \underset{2}{2}, d_{1}, d_{2},-d_{3}, \underset{6}{6}, \ldots, \underset{11}{0},-d_{1} d_{3}, \underset{13}{0}, \ldots, \underset{18}{0}, d_{2} d_{3},-d_{2} d_{3}, \\
0, \ldots, \underset{47}{0}, d_{1} d_{2} d_{3}, \underset{49}{0},-d_{1} d_{2} d_{3}, \underset{51}{0}, \ldots, \underset{53}{0}
\end{array}\right) .
\end{aligned}
$$

Since

$\gamma_{1243}\left(d_{1}, d_{2}, d_{3}, d_{4}\right)$ 


$$
\begin{aligned}
& =\mathfrak{m}\left(\begin{array}{c}
d_{1}, d_{2}, d_{3}, d_{4}, \underset{5}{0}, \ldots, \underset{9}{0}, d_{3} d_{4}, \underset{11}{0}, \ldots, \underset{20}{0}, d_{1} d_{3} d_{4}, \underset{22}{0}, \ldots, \underset{25}{0}, d_{2} d_{3} d_{4}, \underset{27}{0}, \ldots, \underset{30}{0}, \\
d_{1} d_{2} d_{3} d_{4}, \underset{32}{0}, \ldots, \underset{53}{0}
\end{array}\right) \\
& =\mathfrak{m}\left(\begin{array}{c}
d_{1}, d_{2}, d_{3}, d_{4}, d_{1} d_{2}, \underset{6}{0}, \ldots, \underset{9}{0}, d_{3} d_{4}, 0, d_{1} d_{2} d_{3}, \underset{13}{0}, \ldots, \underset{16}{0}, d_{1} d_{2} d_{4}, \underset{18}{0}, \ldots, \underset{20}{0}, \\
d_{1} d_{3} d_{4}, \underset{22}{0}, \ldots, \underset{25}{0}, d_{2} d_{3} d_{4}, \underset{27}{0}, \ldots, \underset{36}{0}, d_{1} d_{2} d_{3} d_{4}, \underset{38}{0}, \ldots, \underset{53}{0}
\end{array}\right), \\
& \left(\gamma_{1243} \frac{\dot{-}}{34} \gamma_{2143}\right)\left(d_{1}, d_{2}, d_{3}\right) \\
& =\mathfrak{m}\left(\begin{array}{c}
0,0, d_{1}, d_{2},-d_{3}, \underset{6}{1}, \ldots, \underset{11}{0},-d_{1} d_{3}, \underset{13}{0}, \ldots, \underset{16}{0},-d_{2} d_{3}, \\
0, \ldots, \underset{30}{0}, d_{1} d_{2} d_{3}, \underset{32}{0}, \ldots, \underset{36}{0},-d_{1} d_{2} d_{3}, \underset{38}{0}, \ldots, \underset{53}{0}
\end{array}\right) .
\end{aligned}
$$

(3.61) and (3.62) imply that

$$
\begin{aligned}
& \left(\left(\gamma_{4123} \frac{\dot{-}}{34} \gamma_{4213}\right) \div\left(\gamma_{1243} \frac{\dot{ }}{34} \gamma_{2143}\right)\right)\left(d_{1}, d_{2}\right) \\
& =\mathfrak{m}\left(\begin{array}{c}
0, \underset{2}{0}, d_{1}, \underset{4}{0}, \ldots, \underset{16}{0}, d_{2}, \underset{18}{0}, d_{2},-d_{2}, \underset{21}{0}, \ldots, \underset{30}{0},-d_{1} d_{2}, \underset{32}{0}, \ldots, \underset{36}{0}, \\
d_{1} d_{2}, \underset{38}{0}, \ldots, \underset{47}{0}, d_{1} d_{2}, \underset{49}{0},-d_{1} d_{2}, \underset{51}{0}, \ldots,{ }_{53}^{0}
\end{array}\right) .
\end{aligned}
$$

(3.60) and (3.63) imply that

$$
\begin{aligned}
& \left(\begin{array}{c}
\left(\left(\gamma_{3412}-\gamma_{3421}\right)-\left(\gamma_{3124}-\gamma_{3214}\right)\right)- \\
\left(\left(\gamma_{4123} \frac{-}{34} \gamma_{4213}\right)-\left(\gamma_{1243}-\gamma_{2143}\right)\right)
\end{array}\right)(d)
\end{aligned}
$$

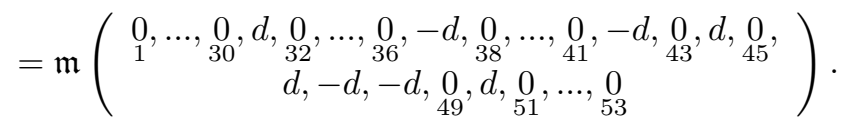

(10) Since

$$
\begin{aligned}
& \gamma_{4132}\left(d_{1}, d_{2}, d_{3}, d_{4}\right) \\
& =\mathfrak{m}\left(\begin{array}{c}
d_{1}, d_{2}, d_{3}, d_{4}, \underset{5}{0} \underset{6}{0}, d_{1} d_{4}, d_{2} d_{3}, d_{2} d_{4}, d_{3} d_{4}, d_{1} d_{2} d_{3}, \underset{12}{0}, \ldots, \underset{18}{0}, d_{1} d_{2} d_{4}, \\
\underset{20}{0}, \ldots, \underset{23}{0}, d_{1} d_{3} d_{4}, \underset{25}{0}, \ldots, \underset{29}{0}, d_{2} d_{3} d_{4}, \underset{31}{0}, \ldots, \underset{48}{0}, d_{1} d_{2} d_{3} d_{4}, \underset{50}{0}, \ldots, \underset{53}{0}
\end{array}\right)
\end{aligned}
$$

and

$$
\begin{aligned}
& \gamma_{4123}\left(d_{1}, d_{2}, d_{3}, d_{4}\right) \\
& =\mathfrak{m}\left(\begin{array}{c}
d_{1}, d_{2}, d_{3}, d_{4}, \underset{5}{0}, \underset{6}{0}, d_{1} d_{4}, \underset{8}{0}, d_{2} d_{4}, d_{3} d_{4}, \underset{11}{0}, \ldots, \underset{18}{0}, d_{1} d_{2} d_{4}, \underset{20}{0}, \ldots, \underset{23}{0,} d_{1} d_{3} d_{4}, \\
0, \ldots, \underset{28}{0}, d_{2} d_{3} d_{4}, \underset{30}{0}, \ldots, \underset{47}{0}, d_{1} d_{2} d_{3} d_{4}, \underset{49}{0}, \ldots, \underbrace{0}_{53}
\end{array}\right),
\end{aligned}
$$

we have

$$
\left(\gamma_{4132} \frac{\cdot}{41} \gamma_{4123}\right)\left(d_{1}, d_{2}, d_{3}\right)
$$




$$
=\mathfrak{m}\left(\begin{array}{c}
d_{2}, \underset{2}{0}, \underset{3}{0}, d_{1}, \underset{5}{0}, \ldots, \underset{7}{0}, d_{3}, \underset{9}{0}, \ldots, \underset{10}{0}, d_{2} d_{3}, \underset{12}{0}, \ldots, \underset{28}{0},-d_{1} d_{3}, d_{1} d_{3}, \\
0, \ldots, \underset{47}{0},-d_{1} d_{2} d_{3}, d_{1} d_{2} d_{3}, \underset{50}{0}, \ldots,{ }_{53}^{0}
\end{array}\right) .
$$

Since

$$
\begin{aligned}
& \gamma_{4321}\left(d_{1}, d_{2}, d_{3}, d_{4}\right) \\
& =\mathfrak{m}\left(\begin{array}{c}
d_{1}, d_{2}, d_{3}, d_{4}, d_{1} d_{2}, d_{1} d_{3}, d_{1} d_{4}, d_{2} d_{3}, d_{2} d_{4}, d_{3} d_{4}, \underset{11}{0}, \ldots, \underset{14}{0}, d_{1} d_{2} d_{3}, \underset{16}{0}, \ldots, \underset{19}{0}, \\
d_{1} d_{2} d_{4}, \underset{21}{0}, \ldots, \underset{24}{0}, d_{1} d_{3} d_{4}, \underset{26}{0}, \ldots, \underset{29}{0}, d_{2} d_{3} d_{4}, \underset{31}{0}, \ldots, d_{1} d_{2} d_{3} d_{4}
\end{array}\right) \\
& \text { and } \\
& \gamma_{4231}\left(d_{1}, d_{2}, d_{3}, d_{4}\right) \\
& =\mathfrak{m}\left(\begin{array}{c}
d_{1}, d_{2}, d_{3}, d_{4}, d_{1} d_{2}, d_{1} d_{3}, d_{1} d_{4}, 0, d_{2} d_{4}, d_{3} d_{4}, 0,0, d_{1} d_{2} d_{3}, \underset{14}{0}, \ldots, \underset{19}{0}, \\
d_{1} d_{2} d_{4}, \underset{21}{0}, \ldots, \underset{24}{0}, d_{1} d_{3} d_{4}, \underset{26}{0}, \ldots, \underset{28}{0}, d_{2} d_{3} d_{4}, \underset{30}{0}, \ldots, \underset{50}{0}, d_{1} d_{2} d_{3} d_{4}, 0, \underset{53}{0}
\end{array}\right), \\
& \left(\gamma_{4321} \dot{4} \gamma_{4231}\right)\left(d_{1}, d_{2}, d_{3}\right) \\
& =\mathfrak{m}\left(\begin{array}{c}
d_{2}, \underset{2}{0}, \underset{3}{0}, d_{1}, \underset{5}{0}, \ldots, \underset{7}{0}, d_{3}, \underset{9}{0}, \ldots, \underset{12}{0},-d_{2} d_{3}, 0, d_{2} d_{3}, \underset{16}{0}, \ldots, \underset{28}{0} \\
-d_{1} d_{3}, d_{1} d_{3}, \underset{31}{0}, \ldots, \underset{50}{0},-d_{1} d_{2} d_{3}, \underset{52}{0}, d_{1} d_{2} d_{3}
\end{array}\right) .
\end{aligned}
$$

(3.65) and (3.66) imply that

$$
\begin{aligned}
& \left(\left(\gamma_{4132}-\gamma_{4123}\right)-\left(\gamma_{4321}-\gamma_{41}\right)\right)\left(d_{1}, d_{2}\right) \\
& =\mathfrak{m}\left(\begin{array}{c}
0, \ldots, 0, d_{1}, 0, \ldots, 0, d_{2}, 0, d_{2}, \underset{12}{0}, 0,-d_{2}, 0, \ldots, 0 \\
3 \\
-d_{1} d_{2}, d_{1} d_{2}, \underset{50}{0}, d_{1} d_{2}, \underset{52}{0},-d_{1} d_{2}
\end{array}\right) .
\end{aligned}
$$

Since

$$
\begin{aligned}
& \gamma_{1324}\left(d_{1}, d_{2}, d_{3}, d_{4}\right) \\
& =\mathfrak{m}\left(\begin{array}{c}
d_{1}, d_{2}, d_{3}, d_{4}, \underset{5}{0}, \underset{6}{0}, \underset{7}{0}, d_{2} d_{3}, \underset{9}{0} \underset{10}{0}, d_{1} d_{2} d_{3}, \underset{12}{0}, \ldots, \underset{26}{0}, d_{2} d_{3} d_{4}, \underset{28}{0}, \ldots, \underset{31}{0}, d_{2} d_{3} d_{4}, \underset{33}{0}, \ldots, \underset{53}{0}
\end{array}\right)
\end{aligned}
$$

and

$$
\gamma_{1234}\left(d_{1}, d_{2}, d_{3}, d_{4}\right)=\mathfrak{m}\left(d_{1}, d_{2}, d_{3}, d_{4}, \underset{5}{0}, \ldots, \underset{53}{0}\right)
$$

we have

$$
\begin{aligned}
& \left(\gamma_{1324}-\gamma_{1234}\right)\left(d_{1}, d_{2}, d_{3}\right) \\
& =\mathfrak{m}\left(\begin{array}{c}
d_{2}, \underset{2}{0}, \underset{3}{2}, d_{1}, \underset{5}{0}, \ldots, \underset{7}{0}, d_{3}, 09,0, d_{2} d_{3}, \underset{12}{0}, \ldots, \underset{26}{0}, \\
d_{1} d_{3}, \underset{28}{0}, \ldots, \underset{31}{0}, d_{1} d_{2} d_{3},{ }_{33}^{0}, \ldots, \underset{53}{0}
\end{array}\right) .
\end{aligned}
$$

Since

$$
\begin{aligned}
& \gamma_{3214}\left(d_{1}, d_{2}, d_{3}, d_{4}\right) \\
& =\mathfrak{m}\left(\begin{array}{c}
d_{1}, d_{2}, d_{3}, d_{4}, d_{1} d_{2}, d_{1} d_{3}, \underset{7}{0}, d_{2} d_{3}, \underset{9}{0}, \ldots, \underset{14}{0}, d_{1} d_{2} d_{3}, 0, d_{1} d_{2} d_{4}, \underset{18}{0}, \ldots, \underset{21}{0}, \\
d_{1} d_{3} d_{4}, \underset{23}{0}, \ldots, \underset{26}{0}, d_{2} d_{3} d_{4}, \underset{28}{0}, \ldots, d_{13} d_{2} d_{3} d_{4}, \underset{45}{0}, \ldots, \underset{53}{0}
\end{array}\right)
\end{aligned}
$$


and

$$
\begin{aligned}
& \gamma_{2314}\left(d_{1}, d_{2}, d_{3}, d_{4}\right) \\
& =\mathfrak{m}\left(\begin{array}{c}
d_{1}, d_{2}, d_{3}, d_{4}, d_{1} d_{2}, d_{1} d_{3}, \underset{7}{0}, \ldots, \underset{12}{0}, d_{1} d_{2} d_{3}, 0,0,0, d_{1} d_{2} d_{4}, \underset{18}{0}, \ldots, \underset{21}{0}, d_{1} d_{3} d_{4}, \\
0, \ldots, \underset{37}{0}, d_{1} d_{2} d_{3} d_{4}, \underset{39}{0}, \ldots, \underset{53}{0}
\end{array}\right), \\
& \left(\gamma_{3214} \underset{41}{-} \gamma_{2314}\right)\left(d_{1}, d_{2}, d_{3}\right) \\
& =\mathfrak{m}\left(\begin{array}{c}
d_{2}, \underset{2}{0}, 0, d_{1}, \underset{5}{0}, \ldots, \underset{7}{0}, d_{3}, \underset{9}{0}, \ldots, \underset{12}{0},-d_{2} d_{3}, \underset{14}{0}, d_{2} d_{3}, \underset{16}{0}, \ldots, \underset{26}{0}, d_{1} d_{3}, \\
0, \ldots,{ }_{37}^{0},-d_{1} d_{2} d_{3}, \underset{39}{0}, \ldots, \underset{43}{0}, d_{1} d_{2} d_{3}, \underset{45}{0}, \ldots, \underset{53}{0}
\end{array}\right) .
\end{aligned}
$$

(3.68) and (3.69) imply that

$$
\begin{aligned}
& \left(\left(\gamma_{1324}-\gamma_{1234}\right)-\left(\gamma_{3214}-\gamma_{2314}\right)\right)\left(d_{1}, d_{2}\right) \\
& =\mathfrak{m}\left(\begin{array}{c}
0, \ldots,{ }_{31}^{0}, d_{1},{ }_{5}, \ldots, \underset{10}{0}, d_{2}, 0, d_{2}, 0,-d_{2}, \underset{16}{0}, \ldots,{ }_{31}^{0}, d_{1} d_{2}, \\
0, \ldots, 0 \\
33
\end{array}\right) .
\end{aligned}
$$

(3.67) and (3.70) imply that

$$
\begin{aligned}
& \left(\begin{array}{c}
\left(\left(\gamma_{4132}-\gamma_{4123}\right)-\left(\gamma_{4321}-\gamma_{4231}\right)\right)- \\
\left(\left(\gamma_{1324} \frac{\dot{-}}{41} \gamma_{1234}\right)-\left(\gamma_{3214} \frac{\dot{-}}{41} \gamma_{2314}\right)\right)
\end{array}\right)(d) \\
& =\mathfrak{m}\left({ }_{1}, \ldots, \underset{31}{0},-d, \underset{33}{0}, \ldots, \underset{37}{0},-d, \underset{39}{0}, \ldots, \underset{43}{0}, d, \underset{45}{0}, \ldots, \underset{47}{0},-d, d, \underset{50}{0}, d, \underset{52}{0},-d\right) .
\end{aligned}
$$

(11) Since

$$
\begin{aligned}
& \gamma_{4213}\left(d_{1}, d_{2}, d_{3}, d_{4}\right) \\
& =\mathfrak{m}\left(\begin{array}{c}
d_{1}, d_{2}, d_{3}, d_{4}, d_{1} d_{2}, \underset{6}{0}, d_{1} d_{4}, \underset{8}{0}, d_{2} d_{4}, d_{3} d_{4}, \underset{11}{0}, d_{1} d_{2} d_{3}, \underset{13}{0}, \ldots, \underset{19}{0}, d_{1} d_{2} d_{4}, \\
\underset{21}{0}, \ldots, \underset{23}{0}, d_{1} d_{3} d_{4}, \underset{25}{0}, \ldots, \underset{28}{0}, d_{2} d_{3} d_{4}, \underset{30}{0}, \ldots, \underset{49}{0}, d_{1} d_{2} d_{3} d_{4}, \underset{51}{0}, \ldots, \underset{53}{0}
\end{array}\right) \\
& \quad \text { and }
\end{aligned}
$$

$\gamma_{4231}\left(d_{1}, d_{2}, d_{3}, d_{4}\right)$

$=\mathfrak{m}\left(\begin{array}{c}d_{1}, d_{2}, d_{3}, d_{4}, d_{1} d_{2}, d_{1} d_{3}, d_{1} d_{4}, \underset{8}{0}, d_{2} d_{4}, d_{3} d_{4}, \underset{11}{0}, \underset{12}{0}, d_{1} d_{2} d_{3}, \underset{14}{0}, \ldots, \underset{19}{0}, \\ d_{1} d_{2} d_{4}, \underset{21}{0}, \ldots, \underset{24}{0}, d_{1} d_{3} d_{4}, \underset{26}{0}, \ldots, \underset{28}{0}, d_{2} d_{3} d_{4}, \underset{30}{0}, \ldots, \underset{50}{0}, d_{1} d_{2} d_{3} d_{4}, \underset{52}{0}, \underset{53}{0}\end{array}\right)$,

we have

$$
\begin{aligned}
& \left(\gamma_{4213} \frac{\cdot}{42} \gamma_{4231}\right)\left(d_{1}, d_{2}, d_{3}\right) \\
& =\mathfrak{m}\left(\begin{array}{c}
0, d_{2}, \underset{3}{0}, d_{1}, \underset{5}{0},-d_{3}, \underset{7}{0}, \ldots, \underset{11}{0}, d_{2} d_{3},-d_{2} d_{3}, \underset{14}{0}, \ldots, \underset{23}{0}, \\
d_{1} d_{3},-d_{1} d_{3}, \underset{26}{0}, \ldots, \underset{49}{0}, d_{1} d_{2} d_{3},-d_{1} d_{2} d_{3}, 0,0
\end{array}\right) .
\end{aligned}
$$

Since

$\gamma_{4132}\left(d_{1}, d_{2}, d_{3}, d_{4}\right)$ 
$=\mathfrak{m}\left(\begin{array}{c}d_{1}, d_{2}, d_{3}, d_{4}, \underset{5}{0}, \underset{6}{0}, d_{1} d_{4}, d_{2} d_{3}, d_{2} d_{4}, d_{3} d_{4}, d_{1} d_{2} d_{3}, \underset{12}{0}, \ldots, \underset{18}{0}, d_{1} d_{2} d_{4}, \\ 0, \ldots, \underset{23}{0}, d_{1} d_{3} d_{4}, \underset{25}{0}, \ldots, \underset{29}{0}, d_{2} d_{3} d_{4}, \underset{31}{0}, \ldots, \underset{48}{0}, d_{1} d_{2} d_{3} d_{4}, \underset{50}{0}, \ldots, \underset{53}{0}\end{array}\right)$

and

$\gamma_{4312}\left(d_{1}, d_{2}, d_{3}, d_{4}\right)$

$=\mathfrak{m}\left(\begin{array}{c}d_{1}, d_{2}, d_{3}, d_{4}, 0, d_{1} d_{3}, d_{1} d_{4}, d_{2} d_{3}, d_{2} d_{4}, d_{3} d_{4}, \underset{11}{0}, \ldots, \underset{13}{0}, d_{1} d_{2} d_{3}, \underset{15}{0}, \ldots, \underset{18}{0}, \underset{20}{0} d_{1} d_{2} d_{4}, \underset{20}{0}, \ldots, \underset{24}{0}, d_{1} d_{3} d_{4}, \underset{26}{0}, \ldots, \underset{29}{0}, d_{2} d_{3} d_{4}, \underset{31}{0}, \ldots, d_{2} d_{3} d_{4}, \underset{53}{0}\end{array}\right)$,

we have

$$
\begin{aligned}
& \left(\gamma_{4132}-\gamma_{42}\right)\left(d_{1}, d_{2}, d_{3}\right) \\
& =\mathfrak{m}\left(\begin{array}{c}
0, d_{2}, \underset{1}{1}, d_{1}, 0,-d_{3}, \underset{7}{0}, \ldots, \underset{10}{0}, d_{2} d_{3}, \underset{12}{0}, \underset{13}{0},-d_{2} d_{3}, \underset{15}{0}, \ldots, \underset{23}{0}, d_{1} d_{3}, \\
-d_{1} d_{3}, \underset{26}{0}, \ldots, \underset{48}{0}, d_{1} d_{2} d_{3}, \underset{50}{0}, \underset{51}{0},-d_{1} d_{2} d_{3}, \underset{53}{0}
\end{array}\right) .
\end{aligned}
$$

(3.72) and (3.73) imply that

$$
\begin{gathered}
\left(\left(\gamma_{4213} \frac{-}{42} \gamma_{4231}\right)-\left(\gamma_{4132}-\gamma_{4312}\right)\right)\left(d_{1}, d_{2}\right) \\
=\mathfrak{m}\left(\begin{array}{c}
0, \ldots, 0, d_{1},{ }_{5}^{0}, \ldots, \underset{10}{0},-d_{2}, d_{2},-d_{2}, d_{2}, \underset{15}{0}, \ldots, \underset{48}{0},-d_{1} d_{2}, \\
d_{1} d_{2},-d_{1} d_{2}, d_{1} d_{2}, \underset{53}{0}
\end{array}\right) .
\end{gathered}
$$

Since

$$
\begin{aligned}
& \gamma_{2134}\left(d_{1}, d_{2}, d_{3}, d_{4}\right) \\
& =\mathfrak{m}\left(\begin{array}{c}
d_{1}, d_{2}, d_{3}, d_{4}, d_{1} d_{2}, \underset{6}{0}, \ldots, \underset{11}{0}, d_{1} d_{2} d_{3}, \underset{13}{0}, \ldots, \underset{16}{0}, d_{1} d_{2} d_{4}, \\
0, \ldots, \underset{35}{0}, d_{1} d_{2} d_{3} d_{4}, \underset{37}{0}, \ldots, \underset{53}{0}
\end{array}\right)
\end{aligned}
$$

and

$$
\begin{aligned}
& \gamma_{2314}\left(d_{1}, d_{2}, d_{3}, d_{4}\right) \\
& =\mathfrak{m}\left(\begin{array}{c}
d_{1}, d_{2}, d_{3}, d_{4}, d_{1} d_{2}, d_{1} d_{3}, \underset{7}{0}, \ldots, \underset{12}{0}, d_{1} d_{2} d_{3}, 0,0,0, d_{1} d_{2} d_{4}, \underset{18}{0}, \ldots, \underset{21}{0}, d_{1} d_{3} d_{4}, \\
0, \ldots,{ }_{37}^{0}, d_{1} d_{2} d_{3} d_{4}, \underset{39}{0}, \ldots, \underset{53}{0}
\end{array}\right),
\end{aligned}
$$

we have

$$
\begin{aligned}
& \left(\gamma_{2134}-\gamma_{42}\right)\left(d_{1}, d_{2}, d_{3}\right) \\
& =\mathfrak{m}\left(\begin{array}{c}
0, d_{2}, \underset{3}{1}, d_{1}, \underset{5}{0},-d_{3}, \underset{7}{0}, \ldots, \underset{11}{0}, d_{2} d_{3},-d_{2} d_{3}, \underset{14}{0}, \ldots, \underset{21}{0},-d_{1} d_{3}, \\
0, \ldots, \underset{35}{0}, d_{1} d_{2} d_{3}, 0,-d_{1} d_{2} d_{3}, \underset{39}{0}, \ldots, \underset{53}{0}
\end{array}\right) .
\end{aligned}
$$

Since

$$
\begin{aligned}
& \gamma_{1324}\left(d_{1}, d_{2}, d_{3}, d_{4}\right) \\
& =\mathfrak{m}\left(\begin{array}{c}
d_{1}, d_{2}, d_{3}, d_{4}, \underset{5}{0}, \underset{6}{0}, \underset{7}{0}, d_{2} d_{3}, \underset{9}{0} \underset{10}{0}, d_{1} d_{2} d_{3}, \underset{12}{0}, \ldots, \underset{26}{0}, d_{2} d_{3} d_{4}, 0,0, \underset{30}{0}, \\
0, d_{1} d_{2} d_{3} d_{4}, \underset{33}{0}, \ldots, \underset{53}{0}
\end{array}\right)
\end{aligned}
$$


and

$$
\begin{aligned}
& \gamma_{3124}\left(d_{1}, d_{2}, d_{3}, d_{4}\right) \\
& =\mathfrak{m}\left(\begin{array}{c}
d_{1}, d_{2}, d_{3}, d_{4}, \underset{5}{0}, d_{1} d_{3}, \underset{7}{0}, d_{2} d_{3}, \underset{9}{0}, \ldots, \underset{13}{0}, d_{1} d_{2} d_{3}, \underset{15}{0}, \ldots, \underset{21}{0}, d_{1} d_{3} d_{4}, \underset{23}{0}, \ldots, \underset{26}{0}, \\
d_{2} d_{3} d_{4}, \underset{28}{0}, \ldots, \underset{41}{0}, d_{1} d_{2} d_{3} d_{4}, \underset{43}{0}, \ldots, \underset{53}{0}
\end{array}\right), \\
& \text { we have } \\
& \left(\gamma_{1324}-\gamma_{42} \gamma_{3124}\right)\left(d_{1}, d_{2}, d_{3}\right) \\
& =\mathfrak{m}\left(\begin{array}{c}
0, d_{2}, \underset{3}{0}, d_{1}, \underset{5}{0},-d_{3}, \underset{7}{0}, \ldots, \underset{10}{0}, d_{2} d_{3}, 0,0,-d_{2} d_{3}, \underset{15}{0}, \ldots, \underset{21}{0}, \\
-d_{1} d_{3}, \underset{23}{0}, \ldots, \underset{31}{0}, d_{1} d_{2} d_{3}, \underset{33}{0}, \ldots, \underset{41}{0},-d_{1} d_{2} d_{3}, \underset{43}{0}, \ldots, \underset{53}{0}
\end{array}\right) .
\end{aligned}
$$

(3.75) and (3.76) imply that

$$
\begin{aligned}
& \left(\left(\gamma_{2134} \frac{-}{42} \gamma_{2314}\right)-\left(\gamma_{1324}-\gamma_{32}\right)\right)\left(d_{1}, d_{2}\right) \\
& =\mathfrak{m}\left(\begin{array}{c}
0, \ldots, 0, d_{1}, \underset{3}{0}, \ldots, \underset{10}{0},-d_{2}, d_{2},-d_{2}, d_{2}, \underset{15}{0}, \ldots, \underset{31}{0},-d_{1} d_{2}, \underset{33}{0}, \ldots, \underset{35}{0}, \\
d_{1} d_{2}, 0,-d_{1} d_{2}, \underset{39}{0}, \ldots, \underset{41}{0}, d_{1} d_{2}, \underset{43}{0}, \ldots, \underset{53}{0}
\end{array}\right) .
\end{aligned}
$$

(3.74) and (3.77) imply that

$$
\begin{aligned}
& \left.\left(\begin{array}{c}
\left(\left(\gamma_{4213}-\gamma_{4231}\right)-\left(\gamma_{4132}-\gamma_{4312}\right)\right)- \\
\left(\left(\gamma_{2134}-\gamma_{42} \gamma_{2314}\right)-\left(\gamma_{1324}-\gamma_{42}\right)\right.
\end{array}\right)\right) \\
& =\mathfrak{m}(d) \\
& (\underset{1}{0}, \ldots, \underset{31}{0}, d, \underset{33}{0}, \ldots, \underset{35}{0},-d, 0, d, \underset{39}{0}, \ldots, \underset{41}{0},-d, \underset{43}{0}, \ldots, \underset{48}{0},-d, d,-d, d, 0
\end{aligned}
$$

(12) Since

$$
\begin{aligned}
& \gamma_{4321}\left(d_{1}, d_{2}, d_{3}, d_{4}\right) \\
& =\mathfrak{m}\left(\begin{array}{c}
d_{1}, d_{2}, d_{3}, d_{4}, d_{1} d_{2}, d_{1} d_{3}, d_{1} d_{4}, d_{2} d_{3}, d_{2} d_{4}, d_{3} d_{4}, \underset{11}{0}, \ldots, \underset{14}{0}, d_{1} d_{2} d_{3}, \underset{16}{0}, \ldots, \underset{19}{0}, \\
d_{1} d_{2} d_{4}, \underset{21}{0}, \ldots, \underset{24}{0}, d_{1} d_{3} d_{4}, \underset{26}{0}, \ldots, \underset{29}{0}, d_{2} d_{3} d_{4}, \ldots, d_{1} d_{2} d_{3} d_{4}
\end{array}\right) \\
& =\mathfrak{m}\left(\begin{array}{c}
d_{1}, d_{2}, d_{3}, d_{4}, \underset{5}{0}, d_{1} d_{3}, d_{1} d_{4}, d_{2} d_{3}, d_{2} d_{4}, d_{3} d_{4}, \underset{11}{0}, \ldots, \underset{13}{0}, d_{1} d_{2} d_{3}, \underset{15}{0}, \ldots, \underset{18}{0}, \underset{20}{0} d_{1} d_{2} d_{4}, \underset{20}{0}, \ldots, d_{24}^{0}, d_{1} d_{3} d_{4}, \underset{26}{0}, \ldots, \underset{29}{0}, d_{2} d_{3} d_{4}, \underset{31}{0}, \ldots, d_{1} d_{2} d_{3} d_{4}, \underset{53}{0}
\end{array}\right), \\
& \left(\gamma_{4321}-\gamma_{43} \gamma_{4312}\right)\left(d_{1}, d_{2}, d_{3}\right) \\
& =\mathfrak{m}\left(\begin{array}{c}
0, \underset{2}{0}, d_{2}, d_{1}, d_{3}, \underset{6}{0}, \ldots, \underset{13}{0},-d_{2} d_{3}, d_{2} d_{3}, \underset{16}{0}, \ldots, \underset{18}{0},-d_{1} d_{3}, d_{1} d_{3}, \\
0, \ldots, \underset{51}{0},-d_{1} d_{2} d_{3}, d_{1} d_{2} d_{3}
\end{array}\right) .
\end{aligned}
$$

Since

$\gamma_{4213}\left(d_{1}, d_{2}, d_{3}, d_{4}\right)$ 


$$
=\mathfrak{m}\left(\begin{array}{c}
d_{1}, d_{2}, d_{3}, d_{4}, d_{1} d_{2}, \underset{6}{0}, d_{1} d_{4}, \underset{8}{0}, d_{2} d_{4}, d_{3} d_{4}, \underset{11}{0}, d_{1} d_{2} d_{3}, \underset{13}{0}, \ldots, \underset{19}{0}, d_{1} d_{2} d_{4}, \\
0, \ldots, \underset{23}{0}, d_{1} d_{3} d_{4}, \underset{25}{0}, \ldots, \underset{28}{0}, d_{2} d_{3} d_{4}, \underset{30}{0}, \ldots, \underset{49}{0}, d_{1} d_{2} d_{3} d_{4}, \underset{51}{0}, \ldots, \underset{53}{0}
\end{array}\right)
$$

and

$$
\begin{aligned}
& \gamma_{4123}\left(d_{1}, d_{2}, d_{3}, d_{4}\right) \\
& =\mathfrak{m}\left(\begin{array}{c}
d_{1}, d_{2}, d_{3}, d_{4}, 0,0, d_{1} d_{4}, 0,0, d_{2} d_{4}, d_{3} d_{4}, \underset{11}{0}, \ldots, \underset{18}{0}, d_{1} d_{2} d_{4}, \underset{20}{0}, \ldots, \underset{23}{0,} d_{1} d_{3} d_{4}, \\
\underset{25}{0}, \ldots, \underset{28}{0}, d_{2} d_{3} d_{4}, \underset{30}{0}, \ldots, \underset{47}{0}, d_{1} d_{2} d_{3} d_{4}, \underset{49}{0}, \ldots, \underset{53}{0}
\end{array}\right), \\
& \left(\gamma_{4213} \frac{\dot{4}}{43} \gamma_{4123}\right)\left(d_{1}, d_{2}, d_{3}\right) \\
& =\mathfrak{m}\left(\begin{array}{c}
0,0, d_{2}, d_{1}, d_{3}, \underset{6}{1}, \ldots, \underset{11}{0}, d_{2} d_{3}, \underset{13}{0}, \ldots, \underset{18}{0},-d_{1} d_{3}, d_{1} d_{3}, \underset{21}{0}, \ldots, \underset{47}{0}, \\
-d_{1} d_{2} d_{3}, \underset{49}{0}, d_{1} d_{2} d_{3}, \underset{51}{0}, \ldots, \underset{53}{0}
\end{array}\right) .
\end{aligned}
$$

(3.79) and (3.80) imply that

$$
\begin{aligned}
& \left(\left(\gamma_{4321}-\gamma_{4312}\right)-\left(\gamma_{4213}-\gamma_{43}\right)\right)\left(d_{1}, d_{2}\right) \\
& =\mathfrak{m}\left(\begin{array}{c}
0, \ldots, 0, d_{1},{ }_{5}, \ldots, \underset{11}{0},-d_{2}, 0,-d_{2}, d_{2}, \underset{16}{0}, \ldots, \underset{47}{0}, d_{1} d_{2}, \underset{49}{0} \\
-d_{1} d_{2}, \underset{51}{0},-d_{1} d_{2}, d_{1} d_{2}
\end{array}\right) .
\end{aligned}
$$

Since

$$
\begin{aligned}
& \gamma_{3214}\left(d_{1}, d_{2}, d_{3}, d_{4}\right) \\
& =\mathfrak{m}\left(\begin{array}{c}
d_{1}, d_{2}, d_{3}, d_{4}, d_{1} d_{2}, d_{1} d_{3}, 0, d_{2} d_{3}, \underset{9}{0}, \ldots, \underset{14}{0}, d_{1} d_{2} d_{3}, 0, d_{1} d_{2} d_{4}, \underset{18}{0}, \ldots, \underset{21}{0}, \\
d_{1} d_{3} d_{4}, \underset{23}{0}, \ldots, \underset{26}{0}, d_{2} d_{3} d_{4}, \underset{28}{0}, \ldots, d_{43} d_{2} d_{3} d_{4}, \underset{45}{0}, \ldots, \underset{53}{0}
\end{array}\right)
\end{aligned}
$$

and

$$
\begin{aligned}
& \gamma_{3124}\left(d_{1}, d_{2}, d_{3}, d_{4}\right) \\
& =\mathfrak{m}\left(\begin{array}{c}
d_{1}, d_{2}, d_{3}, d_{4}, \underset{5}{0}, d_{1} d_{3}, \underset{7}{0}, d_{2} d_{3}, \underset{9}{0}, \ldots, \underset{13}{0}, d_{1} d_{2} d_{3}, \underset{15}{0}, \ldots, \underset{21}{0}, d_{1} d_{3} d_{4}, \underset{23}{0}, \ldots, \underset{26}{0}, d_{23}^{0}, d_{31}^{0}, d_{1} d_{2} d_{3} d_{4}, \underset{43}{0}, \ldots,
\end{array}\right),
\end{aligned}
$$

we have

$$
\begin{aligned}
& \left(\gamma_{3214}-\gamma_{43} \gamma_{3124}\right)\left(d_{1}, d_{2}, d_{3}\right) \\
& =\mathfrak{m}\left(\begin{array}{c}
0, \underset{2}{1}, d_{2}, d_{1}, d_{3}, \underset{6}{0}, \ldots, \underset{13}{0},-d_{2} d_{3}, d_{2} d_{3}, 0, d_{1} d_{3}, \\
0, \ldots, \underset{41}{0},-d_{1} d_{2} d_{3}, 0, d_{1} d_{2} d_{3}, \underset{45}{0}, \ldots, \underset{53}{0}
\end{array}\right) .
\end{aligned}
$$

Since

$$
\begin{aligned}
& \gamma_{2134}\left(d_{1}, d_{2}, d_{3}, d_{4}\right) \\
& =\mathfrak{m}\left(\begin{array}{c}
d_{1}, d_{2}, d_{3}, d_{4}, d_{1} d_{2}, \underset{6}{0}, \ldots, \underset{11}{0}, d_{1} d_{2} d_{3}, \underset{13}{0}, \ldots, \underset{16}{0}, d_{1} d_{2} d_{4}, \\
0, \ldots, \underset{35}{0}, d_{1} d_{2} d_{3} d_{4}, \underset{37}{0}, \ldots, \underset{53}{0}
\end{array}\right)
\end{aligned}
$$


and

$$
\gamma_{1234}\left(d_{1}, d_{2}, d_{3}, d_{4}\right)=\mathfrak{m}\left(d_{1}, d_{2}, d_{3}, d_{4},{ }_{5}, \ldots, 0\right)
$$

we have

$$
\begin{aligned}
& \left(\gamma_{2134}-\gamma_{43}\right) \\
& =\mathfrak{m}\left(d_{1}, d_{2}, \underset{2}{0}, d_{2}, d_{1}, d_{3}, \underset{6}{0}, \ldots, \underset{11}{0}, d_{2} d_{3}, \underset{13}{0}, \ldots, \underset{16}{0}, d_{1} d_{3}, \underset{18}{0}, \ldots, \underset{35}{0}, d_{1} d_{2} d_{3}, \underset{37}{0}, \ldots, \underset{53}{0}\right) .
\end{aligned}
$$

(3.82) and (3.83) imply that

$$
\begin{aligned}
& \left(\left(\gamma_{3214}-\gamma_{4324}\right)-\left(\gamma_{2134}-\gamma_{1234}\right)\right)\left(d_{1}, d_{2}\right) \\
& =\mathfrak{m}\left(\begin{array}{c}
0, \ldots, 0, d_{1}, \underset{5}{1}, \ldots,{ }_{11}^{0},-d_{2}, 0,-d_{2}, d_{2}, \underset{16}{0}, \ldots, \underset{35}{0},-d_{1} d_{2}, \\
0, \ldots, \underset{41}{0},-d_{1} d_{2}, \underset{43}{0}, d_{1} d_{2}, \underset{45}{0}, \ldots, \underset{53}{0}
\end{array}\right) .
\end{aligned}
$$

(3.81) and (3.84) imply that

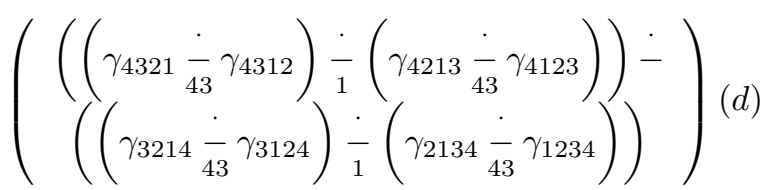

$$
\begin{aligned}
& =\mathfrak{m}(\underset{1}{0}, \ldots, \underset{35}{0}, d, \underset{37}{0}, \ldots, \underset{41}{0}, d, 0,-d, \underset{45}{0}, \ldots, \underset{47}{0}, d, \underset{49}{0},-d, \underset{51}{0},-d, d)
\end{aligned}
$$

(13) By (3.8), (3.15), (3.22), (3.29), (3.36), (3.43), (3.50), (3.57), (3.64), (3.71), (3.78) and (3.85), we have

the left-hand side of the equation (3.1)

$$
\begin{aligned}
& =(\lambda d \cdot \mathfrak{m}(\underset{1}{0}, \ldots, \underset{30}{0},-d, \underset{32}{0},-d, \underset{34}{0}, d, \underset{36}{0}, \ldots, \underset{38}{0},-d, 0, d, \underset{42}{0}, \ldots, \underset{46}{0}, d, \underset{48}{0}, \ldots, \underset{52}{0},-d))+ \\
& (\lambda d \cdot \mathfrak{m}(\underset{1}{0}, \ldots, \underset{30}{0}, d,-d, d,-d, \underset{35}{0}, \ldots, \underset{40}{0},-d, \underset{42}{0}, \ldots \underset{44}{0}, d, \underset{46}{0},-d, \underset{48}{0}, \ldots, \underset{50}{0}, d, \underset{52}{0}, 0))+ \\
& \left(\lambda d \cdot \mathfrak{m}\left({ }_{1}^{0}, \ldots, \underset{31}{0}, d, \underset{33}{0}, d,-d, \underset{36}{0}, \ldots, \underset{38}{0}, d, \underset{40}{0}, \ldots, \underset{44}{0},-d, \underset{46}{0}, \ldots, \underset{50}{0},-d, \underset{52}{0}, d\right)\right)+ \\
& (\lambda d \cdot \mathfrak{m}(\underset{1}{0}, \ldots, \underset{32}{0}, d, \underset{34}{0},-d,-d, d, \underset{38}{0}, d, \underset{40}{0},-d, \underset{42}{0}, \ldots, \underset{45}{0},-d, \underset{47}{0}, \ldots, \underset{51}{0}, d, \underset{53}{0}))+ \\
& (\lambda d \cdot \mathfrak{m}(\underset{1}{0}, \ldots, \underset{34}{0}, d, \underset{36}{0},-d, d,-d, d, \underset{41}{0}, \underset{42}{0},-d, \underset{44}{0} \underset{45}{0}, d, \underset{47}{0}, \underset{48}{0},-d, \underset{50}{0}, \ldots, \underset{53}{0}))+ \\
& (\lambda d \cdot \mathfrak{m}(\underset{1}{0}, \ldots, \underset{32}{0},-d, \underset{34}{0}, \underset{35}{0}, d, \underset{37}{0},-d, \underset{39}{0},-d, d, \underset{42}{0}, d, \underset{44}{0}, \ldots, \underset{48}{0}, d, \underset{50}{0}, 0,-d, \underset{53}{0}))+ \\
& \left(\lambda d \cdot \mathfrak{m}\left(\begin{array}{c}
0, \ldots, \underset{30}{1},-d, \underset{32}{0}, \underset{33}{0}, d, \underset{35}{0}, \ldots, \underset{39}{0}, d, \underset{41}{0}, d,-d, \underset{44}{0},-d, \underset{46}{0}, d, \\
0, \underset{49}{0},-d, \underset{51}{0}, \ldots,{ }_{53}^{0}
\end{array}\right)\right)+ \\
& (\lambda d \cdot \mathfrak{m}(\underset{1}{0}, \ldots, \underset{33}{0},-d \underset{35}{0} \underset{36}{0}, d, \underset{38}{0}, \underset{39}{0},-d, \underset{41}{0}, \underset{42}{0}, d,-d, d,-d, \underset{47}{0}, d, \underset{49}{0}, \ldots, \underset{53}{0}))+ \\
& \left(\lambda d . \mathfrak{m}\left(\begin{array}{c}
0, \ldots, \underset{30}{1}, d, \underset{32}{0}, \ldots, \underset{36}{0},-d, \underset{38}{0}, \ldots, \underset{41}{0},-d, \underset{43}{0}, d, \underset{45}{0}, d,-d,-d, \\
0, d, 0, \ldots,{ }_{53}^{0}
\end{array}\right)\right)+
\end{aligned}
$$




$$
\begin{aligned}
& \left(\lambda d \cdot \mathfrak{m}\left(\underset{1}{0}, \ldots, \underset{31}{0},-d, \underset{33}{0}, \ldots, \underset{37}{0},-d, \underset{39}{0}, \ldots, \underset{43}{0}, d, \underset{45}{0}, \ldots, \underset{47}{0},-d, d, \underset{50}{0}, d, 0,-\frac{d}{53}\right)\right)+ \\
& (\lambda d \cdot \mathfrak{m}(\underset{1}{0}, \ldots, \underset{31}{0}, d, \underset{33}{0}, \ldots, \underset{35}{0},-d, 0, d, \underset{39}{0}, \ldots, \underset{41}{0},-d, \underset{43}{0}, \ldots, \underset{48}{0},-d, d,-d, d, 0))+ \\
& (\lambda d \cdot \mathfrak{m}(\underset{1}{0}, \ldots, \underset{35}{0}, d, \underset{37}{0}, \ldots, \underset{41}{0}, d, 0,-d, \underset{45}{0}, \ldots, \underset{47}{0}, d, 0,-d, 0,-d, d)) \\
& =\lambda d \cdot \mathfrak{m}\left(\begin{array}{c}
0, \ldots, 0,-d+d-d+d,-d+d-d+d, \\
130 \\
-d+d+d-d,-d+d+d-d, d-d-d+d, \\
33 \\
-d+d-d+d, d-d+d-d, d-d-d+d, \\
-d+d+d-d, d-d+d-d, d-d-d+d, \\
39 \\
d-d-d+d,-d+d-d+d,-d+d+d-d, \\
4-d-d+d,-d+d-d+d, d-d+d-d, \\
d-d-d 7 \\
d-d-d+d,-d+d+d-d,-d+d+d-d, \\
48 \\
d-d+d-d, d-d+d-d,-d+d_{53}-d+d \\
51
\end{array}\right) \\
& =\lambda d \cdot \mathfrak{m}\left(\begin{array}{l}
0, \ldots, 0 \\
1
\end{array}\right)
\end{aligned}
$$

Remark 3.5. For our convenience, we display the positions $31-53$ in (3.8), (3.15), (3.22), (3.29), (3.36), (3.43), (3.50), (3.57), (3.64), (3.71), (3.78) and (3.85) as a table:

\begin{tabular}{ccccccccccccc} 
& $\frac{1}{12}$ & $\frac{2}{13}$ & $\frac{3}{14}$ & $\frac{4}{21}$ & $\frac{5}{23}$ & $\frac{6}{24}$ & $\frac{7}{31}$ & $\frac{8}{32}$ & $\frac{9}{34}$ & $\frac{10}{41}$ & $\frac{11}{42}$ & $\frac{12}{43}$ \\
\hline $31 / 1243$ & $-d$ & $d$ & & & & & $-d$ & & $d$ & & & \\
$32 / 1324$ & & $-d$ & $d$ & & & & & & & $-d$ & $d$ & \\
$33 / 1342$ & $-d$ & $d$ & & $d$ & & $-d$ & & & & & & \\
$34 / 1423$ & & $-d$ & $d$ & & & & $d$ & $-d$ & & & & \\
$35 / 1432$ & $d$ & & $-d$ & $-d$ & $d$ & & & & & & & \\
$36 / 2134$ & & & & $-d$ & & $d$ & & & & & $-d$ & $d$ \\
$37 / 2143$ & & & & $d$ & $-d$ & & & $d$ & $-d$ & & & \\
$38 / 2314$ & & & & & $d$ & $-d$ & & & & $-d$ & $d$ & \\
$39 / 2341$ & $-d$ & & $d$ & $d$ & $-d$ & & & & & & & \\
$40 / 2413$ & & & & & $d$ & $-d$ & $d$ & $-d$ & & & & \\
$41 / 2431$ & $d$ & $-d$ & & $-d$ & & $d$ & & & & & & \\
$42 / 3124$ & & & & & & & $d$ & & $-d$ & & $-d$ & $d$ \\
$43 / 3142$ & & & & & $-d$ & $d$ & $-d$ & $d$ & & & & \\
$44 / 3214$ & & & & & & & & $-d$ & $d$ & $d$ & & $-d$ \\
$45 / 3241$ & & $d$ & $-d$ & & & & $-d$ & $d$ & & & & \\
$46 / 3412$ & & & & $-d$ & $d$ & & & $-d$ & $d$ & & & \\
$47 / 3421$ & $d$ & $-d$ & & & & & $d$ & & $-d$ & & & \\
$48 / 4123$ & & & & & & & & $d$ & $-d$ & $-d$ & & $d$ \\
$49 / 4132$ & & & & & $-d$ & $d$ & & & & $d$ & $-d$ & \\
$50 / 4213$ & & & & & & & $-d$ & & $d$ & & $d$ & $-d$
\end{tabular}




\begin{tabular}{cccccccccc}
$51 / 4231$ & & $d$ & $-d$ & & & & & & \\
$52 / 4312$ & & & & $d$ & & $-d$ & & $d$ & $-d$ \\
$53 / 4321$ & $-d$ & & $d$ & & & $-d$ & & $d$ \\
\hline
\end{tabular}

Corollary 3.6. Let $M$ be a microlinear space with

$$
X_{1}, X_{2}, X_{3}, X_{4} \in \mathfrak{X}(M) .
$$

Then, we have

$$
\begin{aligned}
& {\left[X_{1},\left[X_{2},\left[X_{3}, X_{4}\right]\right]\right]+\left[X_{1},\left[X_{3},\left[X_{4}, X_{2}\right]\right]\right]+\left[X_{1},\left[X_{4},\left[X_{2}, X_{3}\right]\right]\right]+} \\
& {\left[X_{2},\left[X_{1},\left[X_{4}, X_{3}\right]\right]\right]+\left[X_{2},\left[X_{3},\left[X_{1}, X_{4}\right]\right]\right]+\left[X_{2},\left[X_{4},\left[X_{3}, X_{1}\right]\right]\right]+} \\
& {\left[X_{3},\left[X_{1},\left[X_{2}, X_{4}\right]\right]\right]+\left[X_{3},\left[X_{2},\left[X_{4}, X_{1}\right]\right]\right]+\left[X_{3},\left[X_{4},\left[X_{1}, X_{2}\right]\right]\right]+} \\
& {\left[X_{4},\left[X_{1},\left[X_{3}, X_{2}\right]\right]\right]+\left[X_{4},\left[X_{2},\left[X_{1}, X_{3}\right]\right]\right]+\left[X_{4},\left[X_{3},\left[X_{2}, X_{1}\right]\right]\right]} \\
& =0 .
\end{aligned}
$$

Proof. Let

$$
\begin{aligned}
& \gamma_{1234}=X_{4} * X_{3} * X_{2} * X_{1}, \gamma_{1243}=\left(X_{3} * X_{4} * X_{2} * X_{1}\right)^{\sigma_{1243}} \\
& \gamma_{1324}=\left(X_{4} * X_{2} * X_{3} * X_{1}\right)^{\sigma_{1324}}, \gamma_{1342}=\left(X_{2} * X_{4} * X_{3} * X_{1}\right)^{\sigma_{1342}} \\
& \gamma_{1423}=\left(X_{3} * X_{2} * X_{4} * X_{1}\right)^{\sigma_{1423}}, \gamma_{1432}=\left(X_{2} * X_{3} * X_{4} * X_{1}\right)^{\sigma_{1432}} \\
& \gamma_{2134}=\left(X_{4} * X_{3} * X_{1} * X_{2}\right)^{\sigma_{2134}}, \gamma_{2143}=\left(X_{3} * X_{4} * X_{1} * X_{2}\right)^{\sigma_{2143}} \\
& \gamma_{2314}=\left(X_{4} * X_{1} * X_{3} * X_{2}\right)^{\sigma_{2314}}, \gamma_{2341}=\left(X_{1} * X_{4} * X_{3} * X_{2}\right)^{\sigma_{2341}} \\
& \gamma_{2413}=\left(X_{3} * X_{1} * X_{4} * X_{2}\right)^{\sigma_{2413}}, \gamma_{2431}=\left(X_{1} * X_{3} * X_{4} * X_{2}\right)^{\sigma_{2431}} \\
& \gamma_{3124}=\left(X_{4} * X_{2} * X_{1} * X_{3}\right)^{\sigma_{3124}}, \gamma_{3142}=\left(X_{2} * X_{4} * X_{1} * X_{3}\right)^{\sigma_{3142}} \\
& \gamma_{3214}=\left(X_{4} * X_{1} * X_{2} * X_{3}\right)^{\sigma_{3214}}, \gamma_{3241}=\left(X_{1} * X_{4} * X_{2} * X_{3}\right)^{\sigma_{3241}} \\
& \gamma_{3412}=\left(X_{2} * X_{1} * X_{4} * X_{3}\right)^{\sigma_{3412}}, \gamma_{3421}=\left(X_{1} * X_{2} * X_{4} * X_{3}\right)^{\sigma_{3421}} \\
& \gamma_{4123}=\left(X_{3} * X_{2} * X_{1} * X_{4}\right)^{\sigma_{4123}}, \gamma_{4132}=\left(X_{2} * X_{3} * X_{1} * X_{4}\right)^{\sigma_{4132}} \\
& \gamma_{4213}=\left(X_{3} * X_{1} * X_{2} * X_{4}\right)^{\sigma_{4213}}, \gamma_{4231}=\left(X_{1} * X_{3} * X_{2} * X_{4}\right)^{\sigma_{4231}} \\
& \gamma_{4312}=\left(X_{2} * X_{1} * X_{3} * X_{4}\right)^{\sigma_{4312}}, \gamma_{4321}=\left(X_{1} * X_{2} * X_{3} * X_{4}\right)^{\sigma_{4321}}
\end{aligned}
$$

with

$$
\begin{aligned}
& \sigma_{1243}=\left(\begin{array}{l}
1234 \\
1243
\end{array}\right), \sigma_{1324}=\left(\begin{array}{l}
1234 \\
1324
\end{array}\right), \sigma_{1342}=\left(\begin{array}{l}
1234 \\
1423
\end{array}\right), \sigma_{1423}=\left(\begin{array}{l}
1234 \\
1342
\end{array}\right), \\
& \sigma_{1432}=\left(\begin{array}{l}
1234 \\
1432
\end{array}\right), \sigma_{2134}=\left(\begin{array}{l}
1234 \\
2134
\end{array}\right), \sigma_{2143}=\left(\begin{array}{l}
1234 \\
2143
\end{array}\right), \sigma_{2314}=\left(\begin{array}{l}
1234 \\
3124
\end{array}\right), \\
& \sigma_{2341}=\left(\begin{array}{l}
1234 \\
4123
\end{array}\right), \sigma_{2413}=\left(\begin{array}{l}
1234 \\
3142
\end{array}\right), \sigma_{2431}=\left(\begin{array}{l}
1234 \\
4132
\end{array}\right), \sigma_{3124}=\left(\begin{array}{l}
1234 \\
2314
\end{array}\right), \\
& \sigma_{3142}=\left(\begin{array}{l}
1234 \\
2413
\end{array}\right), \sigma_{3214}=\left(\begin{array}{l}
1234 \\
3214
\end{array}\right), \sigma_{3241}=\left(\begin{array}{l}
1234 \\
4213
\end{array}\right), \sigma_{3412}=\left(\begin{array}{l}
1234 \\
3412
\end{array}\right), \\
& \sigma_{3421}=\left(\begin{array}{l}
1234 \\
4312
\end{array}\right), \sigma_{4123}=\left(\begin{array}{l}
1234 \\
2341
\end{array}\right), \sigma_{4132}=\left(\begin{array}{l}
1234 \\
2431
\end{array}\right), \sigma_{4213}=\left(\begin{array}{l}
1234 \\
3241
\end{array}\right),
\end{aligned}
$$




$$
\sigma_{4231}=\left(\begin{array}{l}
1234 \\
4231
\end{array}\right), \sigma_{4312}=\left(\begin{array}{l}
1234 \\
3421
\end{array}\right), \sigma_{4321}=\left(\begin{array}{l}
1234 \\
4321
\end{array}\right) .
$$

Then, it is easy to see that

$$
\begin{aligned}
& {\left[X_{1},\left[X_{2},\left[X_{3}, X_{4}\right]\right]\right]=} \\
& \left(\left(\gamma_{1234} \frac{\dot{1}}{12} \gamma_{1243}\right) \div\left(\gamma_{1342} \frac{\dot{1}}{12} \gamma_{1432}\right)\right)-\left(\left(\gamma_{2341} \frac{\dot{1}}{12} \gamma_{2431}\right) \div\left(\gamma_{3421} \frac{\dot{1}}{12} \gamma_{4321}\right)\right), \\
& {\left[X_{1},\left[X_{3},\left[X_{4}, X_{2}\right]\right]\right]=} \\
& \left(\left(\gamma_{1342} \frac{\dot{1}}{13} \gamma_{1324}\right) \div\left(\gamma_{1423} \frac{\dot{1}}{13} \gamma_{1243}\right)\right)-\left(\left(\gamma_{3421} \frac{\dot{1}}{13} \gamma_{3241}\right) \div\left(\gamma_{4231} \frac{\dot{1}}{13} \gamma_{2431}\right)\right), \\
& {\left[X_{1},\left[X_{4},\left[X_{2}, X_{3}\right]\right]\right]=} \\
& \left(\left(\gamma_{1423} \frac{\dot{1}}{14} \gamma_{1432}\right) \div\left(\gamma_{1234} \frac{\dot{1}}{14} \gamma_{1324}\right)\right)-\left(\left(\gamma_{4231} \frac{\dot{-}}{14} \gamma_{4321}\right) \div\left(\gamma_{2341} \frac{\dot{1}}{14} \gamma_{3241}\right)\right), \\
& {\left[X_{2},\left[X_{1},\left[X_{4}, X_{3}\right]\right]\right]=}
\end{aligned}
$$

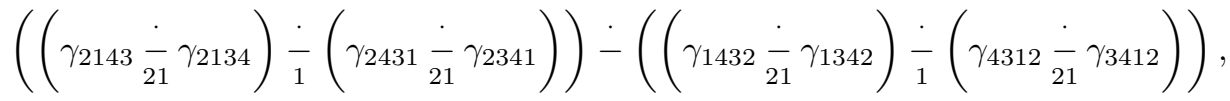

$$
\begin{aligned}
& {\left[X_{2},\left[X_{3},\left[X_{1}, X_{4}\right]\right]\right]=}
\end{aligned}
$$

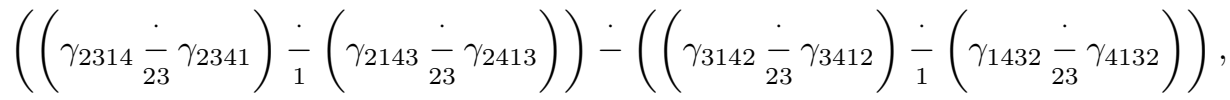

$$
\begin{aligned}
& {\left[X_{2},\left[X_{4},\left[X_{3}, X_{1}\right]\right]\right]=}
\end{aligned}
$$

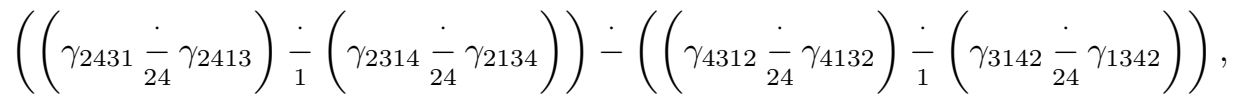

$$
\begin{aligned}
& {\left[X_{3},\left[X_{1},\left[X_{2}, X_{4}\right]\right]\right]=}
\end{aligned}
$$

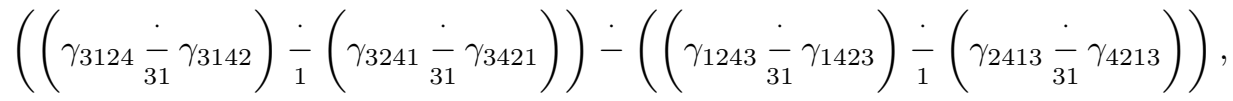

$$
\begin{aligned}
& {\left[X_{3},\left[X_{2},\left[X_{4}, X_{1}\right]\right]\right]=}
\end{aligned}
$$

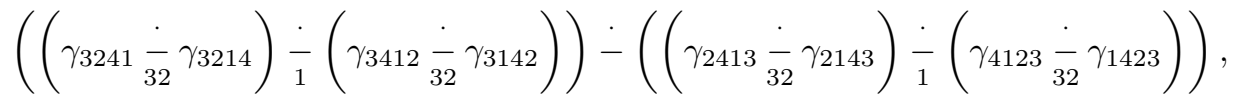

$$
\begin{aligned}
& {\left[X_{3},\left[X_{4},\left[X_{1}, X_{2}\right]\right]\right]=}
\end{aligned}
$$

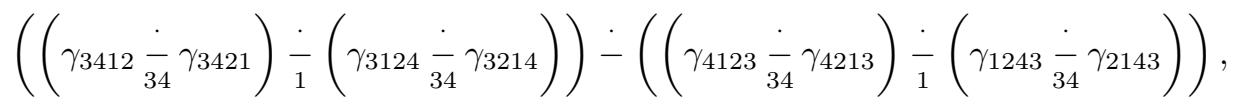

$$
\begin{aligned}
& {\left[X_{4},\left[X_{1},\left[X_{3}, X_{2}\right]\right]\right]=} \\
& \left(\left(\gamma_{4132} \frac{\dot{4}}{41} \gamma_{4123}\right) \div\left(\gamma_{4321} \frac{\dot{-}}{41} \gamma_{4231}\right)\right)-\left(\left(\gamma_{1324} \frac{\cdot}{41} \gamma_{1234}\right) \div\left(\gamma_{3214} \frac{\dot{ }}{41} \gamma_{2314}\right)\right) \\
& {\left[X_{4},\left[X_{2},\left[X_{1}, X_{3}\right]\right]\right]=}
\end{aligned}
$$




$$
\begin{aligned}
& \left(\left(\gamma_{4213} \frac{\dot{4}}{42} \gamma_{4231}\right) \div\left(\gamma_{4132} \frac{\dot{-}}{42} \gamma_{4312}\right)\right)-\left(\left(\gamma_{2134} \frac{\dot{1}}{42} \gamma_{2314}\right) \div\left(\gamma_{1324} \frac{\dot{4}}{42} \gamma_{3124}\right)\right) \\
& {\left[X_{4},\left[X_{3},\left[X_{2}, X_{1}\right]\right]\right]=} \\
& \left(\left(\gamma_{4321} \frac{\dot{4}}{43} \gamma_{4312}\right) \div\left(\gamma_{4213} \frac{\dot{4}}{43} \gamma_{4123}\right)\right)-\left(\left(\gamma_{3214} \frac{\dot{4}}{43} \gamma_{3124}\right) \div\left(\gamma_{2134} \frac{\dot{4}}{43} \gamma_{1234}\right)\right) .
\end{aligned}
$$

\section{REFERENCES}

[1] D. Blessenohl and H. Laue, Generalized Jacobi identities, Note Mat. 8 (1988), 111-121.

[2] A. Kock, Synthetic Differential Geometry, 2nd ed., Cambridge University Press, 2006.

[3] A. Kock and R. Lavendhomme, Strong infinitesimal linearity, with applications to strong difference and affine connections, Cah. Topol. Géom. Différ. 25 (1984), 311-324.

[4] R. Lavendhomme, Basic Concepts of Synthetic Differential Geometry, Kluwer Academic Publishers, 1996.

[5] K. Mackenzie, Proving the Jacobi identity the hard way, in: P. Kielanowski et al. (eds.), Geometric Methods in Physics, Trends in Mathematics, Birkhäuser, 2013, 357-366.

[6] H. Nishimura, Theory of microcubes, Int. J. Theor. Phys. 36 (1997), 1099-1131.

[7] H. Nishimura, General Jacobi identity revisited, Int. J. Theor. Phys. 38 (1999), 2163-2174.

[8] H. Nishimura and T. Osoekawa, General Jacobi identity revisited again, Int. J. Theor. Phys. 46 (2007), 2843-2862.

[9] H. Nishimura, The Jacobi identity beyond Lie algebras, Far East J. Math. Sci. 35 (2009), 33-48.

[10] H. Nishimura, Synthetic differential geometry within homotopy type theory, arXiv math CT/1593662, 2016.

[11] C. Reutenauer, Free Lie Algebras, Oxford: Clarendon Press, 1993.

[12] F. Wever, Über Invarianten in Lieschen Ringen, Math. Ann. 120 (1949), 563-580.

Hirokazu Nishimura, Institute of Mathematics, University of Tsukuba, Tsukuba, Ibaraki 3058571, Japan

e-mail: logic@math.tsukuba.ac.jp 